\title{
DRINFEL'D ALGEBRA DEFORMATIONS, HOMOTOPY COMODULES AND THE ASSOCIAHEDRA
}

\author{
MARTIN MARKL AND STEVE SHNIDER
}

\begin{abstract}
The aim of this work is to construct a cohomology theory controlling the deformations of a general Drinfel'd algebra $A$ and thus finish the program which began in [13], [14]. The task is accomplished in three steps. The first step, which was taken in the aforementioned articles, is the construction of a modified cobar complex adapted to a non-coassociative comultiplication. The following two steps each involves a new, highly non-trivial, construction. The first construction, essentially combinatorial, defines a differential graded Lie algebra structure on the simplicial chain complex of the associahedra. The second construction, of a more algebraic nature, is the definition of a map of differential graded Lie algebras from the complex defined above to the algebra of derivations on the bar resolution. Using the existence of this map and the acyclicity of the associahedra we can define a so-called homotopy comodule structure (Definition 3.3 below) on the bar resolution of a general Drinfel'd algebra. This in turn allows us to define the desired cohomology theory in terms of a complex which consists, roughly speaking, of the bimodule and bicomodule maps from the bar resolution to the modified cobar resolution. The complex is bigraded but not a bicomplex as in the Gerstenhaber-Schack theory for bialgebra deformations. The new components of the coboundary operator are defined via the constructions mentioned above. The results of the paper were announced in [12].
\end{abstract}

\section{InTRODUCTION}

Recall that a Drinfel'd algebra (or a quasi-bialgebra in the original terminology of [3]) is an object $A=(V, \cdot, \Delta, \Phi)$, where $(V, \cdot, \Delta)$ is an associative, not necessarily coassociative, unital and counital k-bialgebra, $\Phi$ is an invertible element of $V^{\otimes 3}$, and the usual coassociativity property is replaced by the condition which we shall refer to as quasi-coassociativity:

$$
(\mathbb{1} \otimes \Delta) \Delta \cdot \Phi=\Phi \cdot(\Delta \otimes \mathbb{1}) \Delta,
$$

where we use the dot - to indicate both the (associative) multiplication on $V$ and the induced multiplication on $V^{\otimes 3}$. Moreover, the validity of the following "pentagon identity" is required:

$$
\left(\mathbb{1}^{2} \otimes \Delta\right)(\Phi) \cdot\left(\Delta \otimes \mathbb{1}^{2}\right)(\Phi)=(1 \otimes \Phi) \cdot(\mathbb{1} \otimes \Delta \otimes \mathbb{1})(\Phi) \cdot(\Phi \otimes 1),
$$

Received by the editors October 3, 1994.

1991 Mathematics Subject Classification. Primary 17B37; Secondary 18 G60.

Key words and phrases. Quasi-bialgebra, formal deformations, multicomplex, associativity constraints.

The first author partially supported by the National Research Counsel, USA.

The second author partially supported by a grant from the Israel Science Foundation administered by the Israel Academy of Sciences and Humanities. 
$1 \in V$ being the unit element and $\mathbb{1}$, the identity map on $V$. If $\epsilon: V \rightarrow \mathbf{k}$ (k being the ground field) is the counit of the coalgebra $(V, \Delta)$, then, by definition, $(\epsilon \otimes \mathbb{1}) \Delta=(\mathbb{1} \otimes \epsilon) \Delta=\mathbb{1}$. A bialgebra is a Drinfel'd algebra with $\Phi=1$.

We have also a natural splitting $V=\bar{V} \oplus \mathbf{k}, \bar{V}:=\operatorname{Ker}(\epsilon)$. We actually do not need to assume the existence of the counit in the paper. All the constructions remain valid, except we would not have the "full cohomology" $H^{*}(A)$ of Definition 6.1 , only the reduced version $\hat{H}^{*}(A)$ of Theorem 6.2 , because the ground field $\mathbf{k}$ would not have a natural $(V, \cdot)$-bimodule structure.

In a recent paper [8], Gerstenhaber and Schack have extended the original Gerstenhaber deformation theory to a deformation theory of bialgebras. We are interested in extending their theory to the case of Drinfel'd algebras. In the past one has always been able to construct a cohomology theory controlling deformations by identifying the complex and extracting the differential from a careful examination of the linearization of the structure axioms. In the geometric theory of deformations, Kodaira and Spencer realized that the linearization of the compatibility equations for the transition functions of a complex structure lead to cohomology with coefficients in the holomorphic tangent sheaf and used it to study deformations of complex structure. On the algebraic side, in the deformation theory of associative algebras, Gerstenhaber realized that if Hochschild cohomology did not already exist, he would have had to invent it. Similarly, in the deformation of associative coalgebras there is a dual theory using the coboundary operator defined by Cartier in the 1950's [2]. The delightful discovery of Gerstenhaber and Schack is that the two theories fit together to form a double complex controlling the deformation of bialgebras and nothing essentially new is needed. However, when we study Drinfel'd algebra deformations, there are some essentially new ingredients and these are not easily derived from a direct study of the structure axioms as we shall see.

After a quick review of the cohomological approach to the deformation theory of bialgebras as defined by Gerstenhaber and Schack we shall describe the rather straightforward extension to deformations of bialgebras (considered as Drinfel' $\mathrm{d}$ algebras with $\Phi=1$ ) in the category of Drinfel'd algebras. Then we will explain the complications which arise when we try to extend the theory to deformations for which the initial structure is a genuine Drinfel' $d$ algebra with the comultiplication which is not assumed to be associative. Basically, nonassociativity significantly complicates the procedure for defining a suitable differential, that is, an endomorphism with square zero, which contains all the basic operations in the structure. This leads us to the new constructions whose definition and properties will occupy us for the rest of the paper. We close this introductory section with an outline of the content of the remaining sections.

Our basic framework is the theory of formal deformations of algebraic structures as formulated by Gerstenhaber [5]. For an introductory presentation, see [15]. If we deform the multiplication the infinitesimal will lie in $\operatorname{Hom}_{\mathbf{k}}(V \otimes V, V)$, the space of k-linear maps $V \otimes V \rightarrow V$. The infinitesimal deformation of the comultiplication lies in $\operatorname{Hom}_{\mathbf{k}}(V, V \otimes V)$. So we introduce a "double complex" and follow the convention of Gerstenhaber and Schack by considering $\operatorname{Hom}_{\mathbf{k}}\left(V^{\otimes q}, V^{\otimes p}\right)$ as having total degree $p+q-1$ and placing it at position $(p, q)$, column $p$, row $q$ as in Figure 1 . The infinitesimal deformation of the multiplication is located at position $(1,2)$, and the fact that the deformed multiplication is associative implies that it is sent to zero by the Hochschild differential represented by the vertical arrow. This is just 


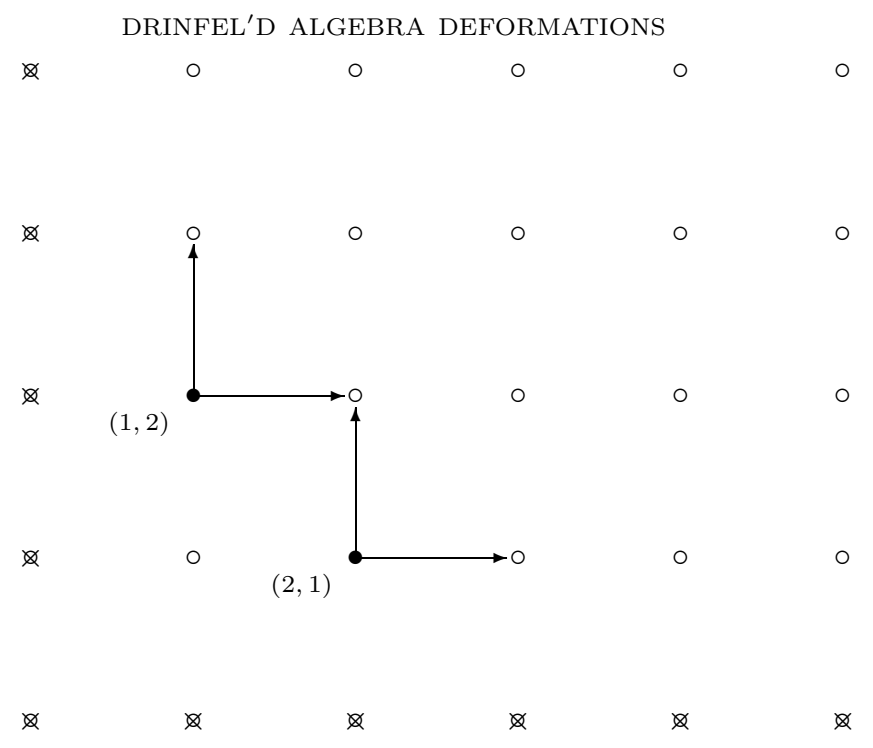

Figure 1. The Gerstenhaber-Schack bialgebra deformation complex. The bottom row and left hand column are deleted (deletion indicated by $\times$ ). The infinitesimal deformation of the multiplication is at position $(1,2)$ and that of the comultiplication is at position $(2,1)$. Vertical arrows represent the Hochschild differential and horizontal arrows represent the Cartier (coalgebra) differential.

the old Gerstenhaber deformation theory for associative algebras, [5]. Dually, the infinitesimal comultiplication is located at $(2,1)$ and the (co)associativity of the deformed comultiplication says that it is sent to zero by the coalgebra differential, introduced by Cartier in the 50's, [2], called sometimes also the co-Hochschild differential, which is represented by the horizontal arrow.

The delightful discovery of Gerstenhaber and Schack is that compatibility of multiplication and comultiplication implies that two contributions at $(2,2)$ (from $(1,2)$ horizontally and from $(2,1)$ vertically) mutually cancel. Thus the sum of the infinitesimal multiplication and comultiplication give a cocycle for the total complex.

From here the theory "follows the book". In the (associative and coassociative) bialgebra deformation theory we must delete the bottom row and leftmost column as in Figure 1. Then an infinitesimal bialgebra deformation, as we have just seen, is a cocycle of total degree two $=p+q-1$ with two components corresponding to multiplication and comultiplication. Two infinitesimal deformations differ by coboundary in this deleted complex if and only if they are related by an infinitesimal "change of coordinates", and hence should be considered equivalent. See Figure 2.

Substituting the putative infinitesimal deformation, i.e. a cocycle of total degree two, in the three structure equations, associativity, coassociativity, and compatibility generates a cocycle of total degree three with three components. See Figure 3. The deformation can be extended to one higher degree in the deformation parameter $t$ if and only if this so-called "obstruction cocycle" is a coboundary.

Now what happens if we start with an associative and coassociative bialgebra, but allow the deformed object to be a Drinfel'd algebra? Amazingly, all that 


\begin{tabular}{|c|c|c|c|c|c|}
\hline$\not$ & 0 & 0 & 0 & 0 & 0 \\
\hline$\not 2$ & $\stackrel{0}{\Lambda}$ & 0 & 0 & 0 & 0 \\
\hline$\not$ & 1 & -0 & 0 & 0 & 0 \\
\hline$\not$ & $\not 1$ & $\not$ & $\not$ & $\not$ & $\not$ \\
\hline
\end{tabular}

Figure 2. Two infinitesimal deformations are equivalent if they differ by a coboundary in the restricted complex.

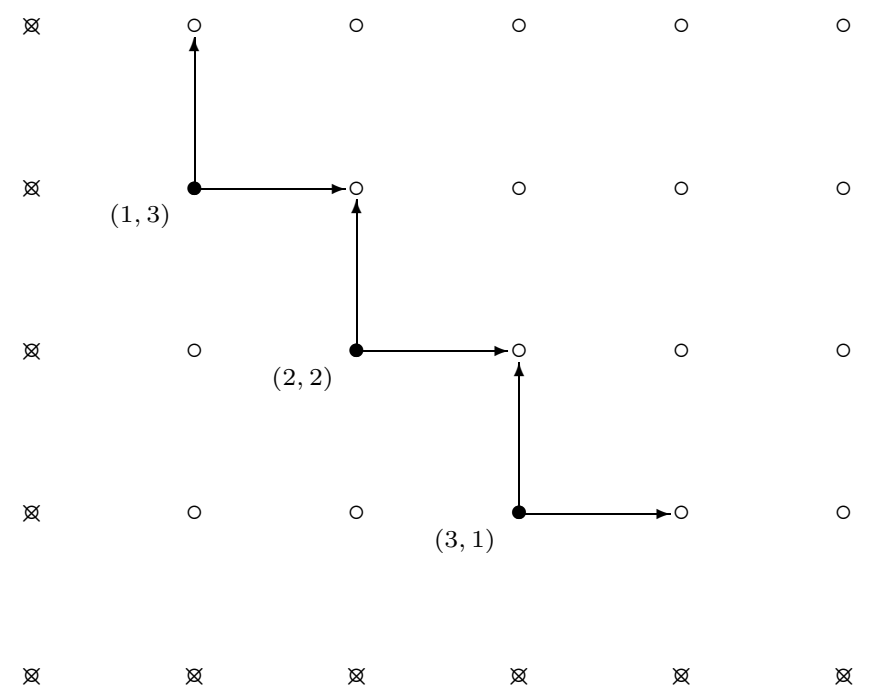

Figure 3. The obstruction to extending the infinitesimal one higher power in $t$ is a cocycle of total degree three as drawn. If this cocycle is a coboundary, the extension is possible.

has to be done is to include the bottom row! Indeed, the horizontal arrow from position $(2,1)$ may no longer send the infinitesimal comultiplication to zero, since the comultiplication need not be coassociative. The infinitesimal deformation now includes the infinitesimal of $\Phi$ which lies in $V^{\otimes 3}=\operatorname{Hom}_{\mathbf{k}}\left(\mathbf{k}, V^{\otimes 3}\right)$ and so is located at $(3,0)$. The fact that the comultiplication is coassociative up to conjugation by $\Phi$ translates, at the infinitesimal level, into the assertion that the contributions at $(3,1)$ coming horizontally from $(2,1)$ and vertically from $(3,0)$ cancel. The pentagon identity on $\Phi$ considered as a fourth structure equation introduces a fourth component to the obstruction cocycle. At the infinitesimal level the pentagon identity implies that the image of the infinitesimal of $\Phi$ in position $(4,0)$ under the coalgebra differential vanishes. The situation is as described by Figure 4 . 


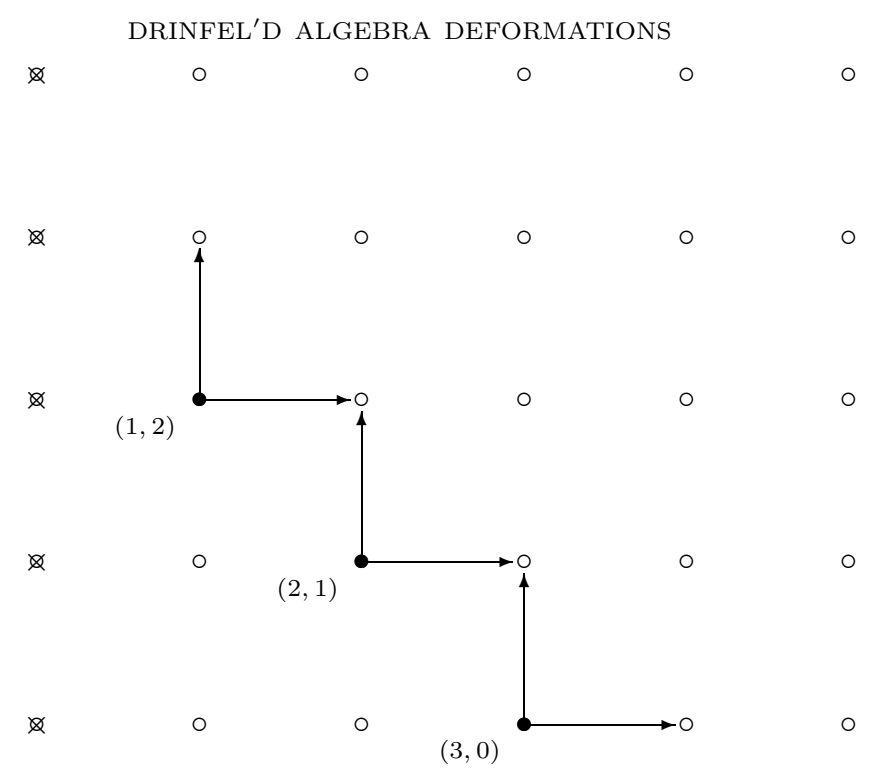

Figure 4. Bialgebra to Drinfel'd algebra deformation. The bottom row is enabled but not the left column. The deformed comultiplication need not be coassociative, so the horizontal arrow at $(2,1)$ need not give zero. The infinitesimal of $\Phi$ lies at position $(3,0)$.

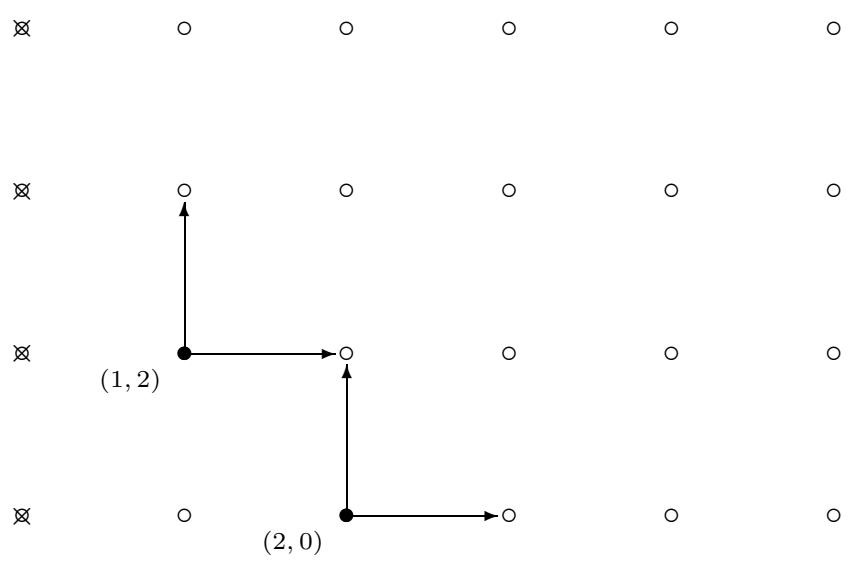

Figure 5. Equivalence in bialgebra to Drinfel'd algebra deformations. The infinitesimal of the twist lies at position $(2,0)$.

For Drinfel'd algebras there is a broader definition of equivalence. One must allow not change of coordinates, but also a twisting (see the original paper [3] for the definition). The infinitesimal of the twisting is located at position $(2,0)$. So allowing this modified infinitesimal equivalence, two three-cocycles of the total complex give equivalent deformations if they differ by a total coboundary as indicated in Figure 5 .

Once again, there is a quadratic expression in a putative infinitesimal deformation which is a cocycle of total degree three. The vanishing of the corresponding total cohomology class determines whether or not the infinitesimal deformation can be extended to a power series of one higher order in $t$. See Figure 6 . 


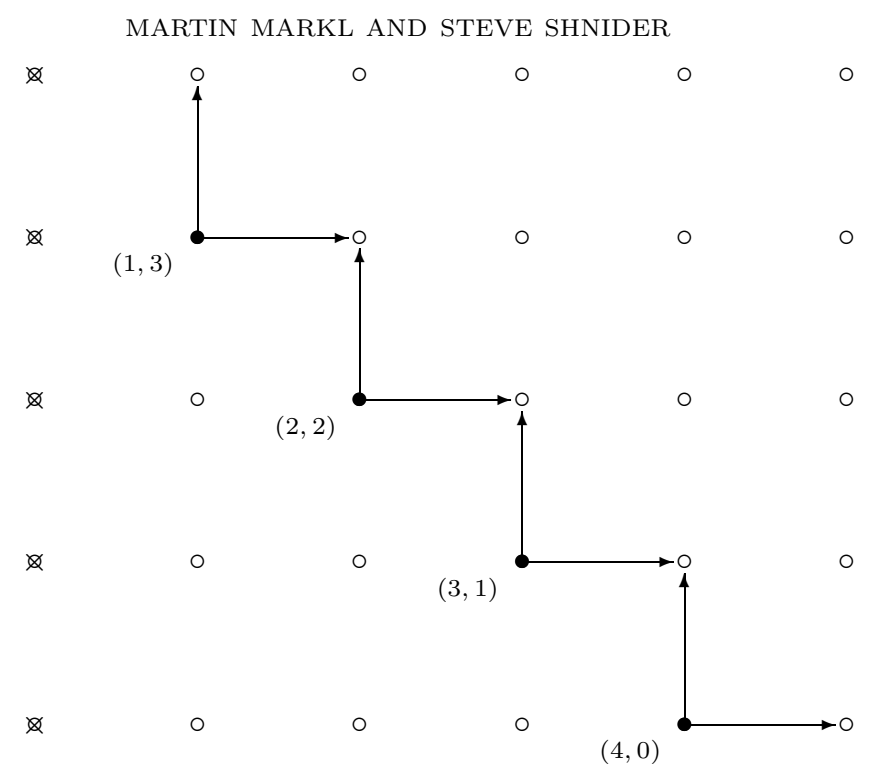

Figure 6. The obstruction to extending the infinitesimal one higher power in $t$ is a cocycle of total degree three.

This, in outline, is the cohomological approach to the theory of deformations of bialgebras, when the deformed object is a Drinfel'd algebra. For details see [15]. What happens when the undeformed structure is that of a Drinfel'd algebra and the comultiplication is not necessarily associative? The first problem is that the standard construction of the cobar differential does not have square zero. We must consider the maps $(\Delta \otimes \mathbb{1}) \Delta$ and $(\mathbb{1} \otimes \Delta) \Delta$ as taking values in different three-fold tensor products defined by different bracketings. After distinguishing between different bracketings we use the quasi-coassociativity condition (1) to define an equivalence between them. This leads to a modified cobar resolution whose construction is reviewed in Section 2.

There is no need to change the definition of the bar resolution since a Drinfel'd algebra is, in particular, an associative algebra. However, the complex which captures both the algebra and coalgebra structures consists of the $A$-bimodule, $A$-bicomodule homomorphisms from the bar resolution to the cobar resolution and this requires the definition of an $A$-bicomodule structure on the bar resolution and an $A$-bimodule structure on the modified cobar resolution. Once again there are some surprises. The $A$-bimodule structure on the modified cobar resolution presents no difficulties, but the $A$-bicomodule structure on the bar resolution does. In general the definition of comodules over a Drinfel' $d$ algebra requires special attention since the usual compatibility conditions for comodules are based on the assumption that the comultiplication is coassociative. There is a natural extension of the definition of an $A$-(bi)comodule which involves using $\Phi$ to relate the different placement of parentheses. The new ingredient which complicates (we think "enriches") our theory is that the obvious definition of a comodule structure on the bar complex, the one which reduces to the standard one for a bialgebra, does not satisfy the required compatibility condition involving $\Phi$. Thus we are led to introduce the notion of a homotopy comodule structure in Section 3.

This definition involves a hierarchy of operators generalizing the standard comodule structure operators. The first new operator can be defined directly but 
the complications involved in defining the higher operators become unmanageable very quickly without some auxiliary construction. The key here is the definition of a differential graded Lie algebra structure on the simplicial chain complex of the associahedra as given in Section 4. This allows us to keep track of the intricate combinatorics related to nonassociativity.

In Section 5 we define a homomorphism of differential graded Lie algebras from the complex defined in Section 4 to the derivations of the bar complex. In Section 6 we describe the relation of all the preceding constructions to the deformation theory of Drinfel'd algebras. As an example we consider the Drinfel'd quasi-Hopf quantization of $U(\mathcal{G})$ for a simple Lie algebra $\mathcal{G}$, see [3] . The last section contains the proofs of some technical lemmas.

\section{Algebraic preliminaries}

All algebraic objects in the paper will be considered over a fixed field $\mathbf{k}$ of characteristic zero. We will systematically use the Koszul sign convention meaning that whenever we commute two "things" of degrees $p$ and $q$, respectively, we multiply by the sign $(-1)^{p q}$. For a graded vector space $M$, let $\uparrow M$ (resp. $\downarrow M$ ) be the suspension (resp. the desuspension) of $M$, i.e. the graded vector space defined by $(\uparrow M)_{p}:=M_{p-1}\left(\operatorname{resp} . \quad(\downarrow M)_{p}:=M_{p+1}\right)$. We have the obvious natural isomorphisms $\uparrow: M \rightarrow \uparrow M$ and $\downarrow: M \rightarrow \downarrow M$.

For a $(V, \cdot)$-module $N$, define the following generalization of the $M$-construction of [13, par. 3]. Let $F^{*}=\bigoplus_{n \geq 0} F^{n}$ be the free unitary nonassociative k-algebra generated by $N$, graded by the length of words. The space $F^{n}$ is the direct sum of copies of $N^{\otimes n}$ over the set $\mathrm{Br}_{n}$ of full bracketings of $n$ symbols, $F^{n}=\bigoplus_{u \in \mathrm{Br}_{n}} N_{u}^{\otimes n}$. For example, $F^{0}=\mathbf{k}, F^{1}=N, F^{2}=N^{\otimes 2}, F^{3}=N_{(\bullet \bullet) \bullet}^{\otimes 3} \oplus N_{\bullet(\bullet \bullet)}^{\otimes 3}$, etc. The algebra $F^{*}$ admits a natural left action, $(a, f) \mapsto a \bullet f$, of $(V, \cdot)$ given by the rules:

i. on $F^{0}=\mathbf{k}$, the action is given by the augmentation $\epsilon$,

ii. on $F^{1}=N$, the action is given by the action of $V$ on $N$ and

iii. $a \bullet(f \star g)=\sum\left(\Delta^{\prime}(a) \bullet f\right) \star\left(\Delta^{\prime \prime}(a) \bullet g\right)$,

where $\star$ stands for the multiplication in $F^{*}$ and we use the notation $\Delta(a)=$ $\sum \Delta^{\prime}(a) \otimes \Delta^{\prime \prime}(a)$. The right action $(f, b) \mapsto f \bullet b$ is defined by similar rules. These actions define on $F^{*}$ the structure of a $(V, \cdot)$-bimodule.

Let $\sim$ be the relation on $F^{*} \star$-multiplicatively generated by the expressions of the form

$$
\sum\left(\left(\Phi_{1} \bullet x\right) \star\left(\left(\Phi_{2} \bullet y\right) \star\left(\Phi_{3} \bullet z\right)\right)\right) \sim \sum\left(\left(\left(x \bullet \Phi_{1}\right) \star\left(y \bullet \Phi_{2}\right)\right) \star\left(z \bullet \Phi_{3}\right)\right),
$$

where $\Phi=\sum \Phi_{1} \otimes \Phi_{2} \otimes \Phi_{3}$ and $x, y, z \in F^{*}$. Put $\odot(N):=F / \sim$. Just as in [13, Proposition 3.2] one proves that the -action induces on $\odot(N)$ the structure of a $(V, \cdot)$-bimodule (denoted again by $\bullet$ ) and that $\star$ induces on $\odot(N)$ a nonassociative multiplication denoted by $\odot$. The operations are related by

$$
\begin{aligned}
a \bullet(f \odot g) & =\sum\left(\Delta^{\prime}(a) \bullet f\right) \odot\left(\Delta^{\prime \prime}(a) \bullet g\right) \quad \text { and } \\
(f \odot g) \bullet b & =\sum\left(f \bullet \Delta^{\prime}(b)\right) \odot\left(g \bullet \Delta^{\prime \prime}(b)\right),
\end{aligned}
$$


for $a, b \in V$ and $f, g \in \odot(N)$. The multiplication $\odot$ is quasi-associative in the sense

$$
\sum\left(\Phi_{1} \bullet x\right) \odot\left(\left(\Phi_{2} \bullet y\right) \odot\left(\Phi_{3} \bullet z\right)\right)=\sum\left(\left(x \bullet \Phi_{1}\right) \odot\left(y \bullet \Phi_{2}\right)\right) \odot\left(z \bullet \Phi_{3}\right) .
$$

Since the defining relations (3) are homogeneous with respect to length, the grading of $F^{*}$ induces on $\odot(N)$ the grading $\bigodot^{*}(N)=\bigoplus_{i \geq 0} \bigodot^{i}(N)$. If $N$ itself is a graded vector space, we have also a second grading, $\operatorname{deg}\left(x_{1} \odot x_{2} \odot \cdots \odot x_{k}\right)=\sum \operatorname{deg}\left(x_{i}\right)$. Denote this grading by $\odot(N)=\bigoplus_{j} \odot(N)^{j}$. It coincides with the first grading if $N$ is concentrated in degree 1.

Let $\operatorname{Der}_{V}^{n}(\odot N)$ denote the set of $(V, \cdot)$-linear derivations of degree $n$ (relative to the second grading) of the (nonassociative) graded algebra $\odot(N)^{*}$. One sees immediately that there is a one-to-one correspondence between the elements $\theta \in$ $\operatorname{Der}_{V}^{n}(\odot N)$ and $(V, \cdot)$-linear homogeneous degree $n$ maps $f: N^{*} \rightarrow \odot(N)^{*}$.

For the Drinfel'd algebra itself, $\Delta: V \rightarrow V \otimes V=V \odot V$ determines an element $d_{C} \in \operatorname{Der}_{V}^{1}(\odot V)$. By the quasi-coassociativity relation (1) we have $(\mathbb{1} \otimes \Delta) \Delta(v)=$ $\Phi \cdot(\Delta \otimes \mathbb{1}) \Delta(v) \cdot \Phi^{-1}$ and the defining relation of the $M$-construction (3) thus gives $(\Delta \odot \mathbb{1}) \Delta(v)=(\mathbb{1} \odot \Delta) \Delta(v)$ for $v \in V$, therefore $d_{C}^{2}=0$ follows from the same line of argument as in the coassociative case. Then $\left(\odot V, d_{C}\right)$ is the modified cobar resolution. For more details see $[13,14]$.

If $N=X \oplus Y$, then $\odot(X \oplus Y)$ is naturally bigraded, $\bigodot^{*, *}(X \oplus Y)=$ $\bigoplus_{i, j \geq 0} \bigodot^{i, j}(X \oplus Y)$, this bigrading being defined by saying that a monomial $w$ belongs to $\bigodot^{i, j}(X \oplus Y)$ if there are exactly $i$ (resp. $j$ ) occurrences of the elements of $X$ (resp. $Y$ ) in $w$. If $X, Y$ are graded vector spaces, then there is a second bigrading just as above.

Let $\left(\mathcal{B}(V), d_{\mathcal{B}}\right)$ be the (two-sided) normalized bar resolution of the algebra $(V, \cdot)$ (see $[9$, Chapter X]), but considered with the opposite grading. This means that $\mathcal{B}(V)$ is the graded space, $\mathcal{B}(V)=\bigoplus_{n \leq 1} \mathcal{B}_{n}(V)$, where $\mathcal{B}_{1}(V)=V, \mathcal{B}_{0}(V)=V \otimes V$, and for $n \leq-1, \mathcal{B}_{n}(V)$ is the free $(V, \cdot)$-bimodule on $\bar{V}^{\otimes(-n)}$, i.e. the vector space $V \otimes \bar{V}^{\otimes(-n)} \otimes V$ with the action of $(V, \cdot)$ given by

$$
\begin{aligned}
& u \cdot\left(a_{0} \otimes \cdots \otimes a_{-n+1}\right):=\left(u \cdot a_{0} \otimes \cdots \otimes a_{-n+1}\right) \quad \text { and } \\
& \left(a_{0} \otimes \cdots \otimes a_{-n+1}\right) \cdot w:=\left(a_{0} \otimes \cdots \otimes a_{-n+1} \cdot w\right)
\end{aligned}
$$

for $u, v, a_{0}, a_{-n+1} \in V$ and $a_{1}, \ldots, a_{-n} \in \bar{V}$. If we use the more compact notation (though a nonstandard one), writing $\left(a_{0}|\cdots| a_{-n+1}\right)$ instead of $a_{0} \otimes \cdots \otimes a_{-n+1}$, the differential $d_{\mathcal{B}}: \mathcal{B}_{n}(V) \rightarrow \mathcal{B}_{n+1}(V)$ is defined as

$$
d_{\mathcal{B}}\left(a_{0}|\cdots| a_{-n+1}\right):=\sum_{0 \leq i \leq-n}(-1)^{i}\left(a_{0}|\cdots| a_{i} \cdot a_{i+1}|\cdots| a_{-n+1}\right)
$$

Here, as is usual in this context, we make no distinction between the elements of $V / \mathbf{k} \cdot 1$ and their representatives in $\bar{V}$. We use the same convention throughout all the paper.

Notice that the differential $d_{\mathcal{B}}$ is a $(V, \cdot)$-linear map. We have two more $(V, \cdot)$ linear maps, namely the 'coactions' $\lambda: \mathcal{B}(V) \rightarrow V \odot \mathcal{B}(V)$ and $\rho: \mathcal{B}(V) \rightarrow \mathcal{B}(V) \odot V$ 
given by

$$
\begin{aligned}
\lambda\left(a_{0}|\cdots| a_{-n+1}\right) & :=\sum \Delta^{\prime}\left(a_{0}\right) \cdots \Delta^{\prime}\left(a_{-n+1}\right) \odot\left(\Delta^{\prime \prime}\left(a_{0}\right)|\cdots| \Delta^{\prime \prime}\left(a_{-n+1}\right)\right), \text { and } \\
\rho\left(a_{0}|\cdots| a_{-n+1}\right) & :=\sum\left(\Delta^{\prime}\left(a_{0}\right)|\cdots| \Delta^{\prime}\left(a_{-n+1}\right)\right) \odot \Delta^{\prime \prime}\left(a_{0}\right) \cdots \Delta^{\prime \prime}\left(a_{-n+1}\right) .
\end{aligned}
$$

A simple computation shows that these "coactions" are compatible with the differential in the sense that

$$
\left(\mathbb{1} \odot d_{\mathcal{B}}\right)(\lambda)=\lambda\left(d_{\mathcal{B}}\right) \text { and }\left(d_{\mathcal{B}} \odot \mathbb{1}\right)(\rho)=\rho\left(d_{\mathcal{B}}\right) .
$$

Most of the constructions presented here would work also when we use the unnormalized bar resolution instead of the normalized one, but in the normalized case we have better control over the objects of Sublemma 5.11.

\section{Homotopy COMOdule StRUCture ON THE BAR RESOlUtion}

Recall that a (differential) bicomodule over a coassociative coalgebra $B=(V, \Delta)$ is a (differential) graded vector space $\left(M, d_{M}\right), \operatorname{deg}\left(d_{M}\right)=1$, together with two homogeneous degree zero linear maps $\lambda: M \rightarrow V \otimes M$ and $\rho: M \rightarrow M \otimes V$ (the coactions) such that

$$
\left(\mathbb{1} \otimes d_{M}\right)(\lambda)=\lambda\left(d_{M}\right),\left(d_{M} \otimes \mathbb{1}\right)(\rho)=\rho\left(d_{M}\right),
$$

$(\Delta \otimes \mathbb{1})(\lambda)=(\mathbb{1} \otimes \lambda)(\lambda),(\lambda \otimes \mathbb{1})(\rho)=(\mathbb{1} \otimes \rho)(\lambda)$ and $(\mathbb{1} \otimes \Delta)(\rho)=(\rho \otimes \mathbb{1})(\rho)$. Here, of course, $V$ is considered as a graded vector space concentrated in degree zero.

We formulate the following lemma as a motivation for the definition of the homotopy comodule structure given in Definition 3.3. The proof is an easy exercise and we leave it to the reader.

Lemma 3.1. Let $\left(M, d_{M}\right)$ be a differential graded vector space and let $\otimes(V, M):=$ $\otimes(\uparrow V \bigoplus \uparrow M)$. Let $(\lambda, \rho)$ be a $(V, \Delta)$-bicomodule structure on $\left(M, d_{M}\right)$ and define $D \in \operatorname{Der}^{1}(\otimes(V, M))$ as $D:=D_{-1}+D_{0}$, where

$$
\begin{aligned}
\left.D_{-1}\right|_{\uparrow M}:=\uparrow d_{M} \downarrow,\left.D_{-1}\right|_{\uparrow V} & :=0, \\
\left.D_{0}\right|_{\uparrow M}:=(\uparrow \otimes \uparrow)(\lambda+\rho)(\downarrow), \text { and }\left.D_{0}\right|_{\uparrow V}: & =(\uparrow \otimes \uparrow)(\Delta)(\downarrow) .
\end{aligned}
$$

Then $D^{2}=0$.

The above formula defines a one-to-one correspondence between $(V, \Delta)$-bicomodule structures on $\left(M, d_{M}\right)$ and differentials $D=D_{-1}+D_{0} \in \operatorname{Der}^{1}(\otimes(V, M))$, where the components $D_{i}$ are of degree $i+1$ relative to the first grading in $V$ (the number of factors of $V$ ) and satisfy the conditions

$$
\left.D_{-1}\right|_{\uparrow V}=0 \text {, and }\left.D_{0}\right|_{\uparrow V}=(\uparrow \otimes \uparrow)(\Delta)(\downarrow) .
$$

We think that some comments on how to interpret the formulas of the lemma are in order. Let $m \in M$ be a homogeneous element, $\operatorname{deg}(m)=p$. We write as usual $\lambda(m)=\sum \lambda^{\prime}(m) \otimes \lambda^{\prime \prime}(m)$ and $\rho(m)=\sum \rho^{\prime}(m) \otimes \rho^{\prime \prime}(m)$ with $\lambda^{\prime}(m), \rho^{\prime \prime}(m) \in V$ and $\lambda^{\prime \prime}(m), \rho^{\prime}(m) \in M_{p}$. Then

$$
\begin{aligned}
D_{0}(\uparrow m) & =(\uparrow \otimes \uparrow)(\lambda+\rho)(m) \\
& =(\uparrow \otimes \uparrow)\left(\lambda^{\prime}(m) \otimes \lambda^{\prime \prime}(m)\right)+(\uparrow \otimes \uparrow)\left(\rho^{\prime}(m) \otimes \rho^{\prime \prime}(m)\right) \\
& =\uparrow \lambda^{\prime}(m) \otimes \uparrow \lambda^{\prime \prime}(m)+(-1)^{p}\left(\uparrow \rho^{\prime}(m) \otimes \uparrow \rho^{\prime \prime}(m)\right) .
\end{aligned}
$$

Here the sign $(-1)^{p}$ is due to the commuting of $\rho^{\prime}(m)$ (a "thing" of degree $p$ ) and the map $\uparrow$ (a "thing" of degree 1). It is useful sometimes to identify an element 
with its image under the map $\uparrow$ (or $\downarrow$ ). Under this identification, the above equation can be written as

$$
D_{0}(m)=\lambda(m)+(-1)^{(|m|+1)} \rho(m) .
$$

Notice the unexpected $\operatorname{sign}(-1)^{(|m|+1)}$ which is due to the fact that $m$ here represents $\uparrow m$, and $|\uparrow m|=p+1$. We see that we must be extremely careful when using this shorthand, especially as far as the sign issue is concerned.

Suppose for the moment that $A=(V, \cdot, \Delta, \Phi)$ is a Drinfel'd algebra with $\Phi=1$. In this case $(V, \cdot, \Delta)$ is an ordinary (associative and coassociative) bialgebra and $\odot$ can be replaced by $\otimes$ everywhere in the paragraph defining the coactions on $\mathcal{B}(V)$. One easily verifies that $\lambda: \mathcal{B}(V) \rightarrow V \otimes \mathcal{B}(V)$ and $\rho: \mathcal{B}(V) \rightarrow \mathcal{B}(V) \otimes V$ describe a $(V, \Delta)$-bicomodule structure on the differential space $\left(\mathcal{B}(V), d_{\mathcal{B}}\right)$. Lemma 3.1 then gives a differential $D=D_{-1}+D_{0} \in \operatorname{Der}^{1}(\otimes(V, \mathcal{B}(V))$.

In the general case with $\Phi \neq 1$ all the above definitions make sense, but we need not have $D^{2}=0$. Let us discuss this situation more carefully. Put $\odot(V, \mathcal{B}(V)):=$ $\odot(\uparrow V \oplus \uparrow \mathcal{B}(V))$ and define the derivations $D_{-1}, D_{0} \in \operatorname{Der}^{1}(\odot(V, \mathcal{B}(V)))$ by

$$
\begin{gathered}
\left.D_{-1}\right|_{\uparrow \mathcal{B}(V)}:=\uparrow d_{\mathcal{B}} \downarrow,\left.D_{-1}\right|_{\uparrow V}:=0, \\
\left.D_{0}\right|_{\uparrow \mathcal{B}(V)}:=(\uparrow \odot \uparrow)(\lambda+\rho)(\downarrow), \text { and }\left.D_{0}\right|_{\uparrow V}:=(\uparrow \odot \uparrow)(\Delta)(\downarrow) .
\end{gathered}
$$

Because of the $(V, \cdot)$-linearity of the maps $d_{\mathcal{B}}, \lambda$ and $\rho$, the derivations $D_{-1}$ and $D_{0}$ are each $(V, \cdot)$-linear, $D_{-1}, D_{0} \in \operatorname{Der}_{V}^{1}(\bigodot(V, \mathcal{B}(V)))$ and clearly

$$
\begin{gathered}
D_{-1}\left(\bigodot(V, \mathcal{B}(V))^{i, j}\right) \subset \bigodot(V, \mathcal{B}(V))^{i, j+1} \text { and } \\
D_{0}\left(\bigodot(V, \mathcal{B}(V))^{i, j}\right) \subset \bigodot(V, \mathcal{B}(V))^{i+1, j}
\end{gathered}
$$

for any $n \leq-1$ and $i, j \geq 0$. The desired condition $D^{2}=0$ requires

$$
D_{-1}^{2}=0, D_{-1} D_{0}+D_{0} D_{-1}=0 \text { and } D_{0}^{2}=0 .
$$

Lemma 3.2. In the situation above, we have two of the three conditions for $D^{2}=$ 0 , namely,

$$
D_{-1}^{2}=0 \text { and the graded commutator } D_{-1} D_{0}+D_{0} D_{-1}=0 .
$$

Proof. It is enough to verify the conditions on the generators $\uparrow V$ and $\uparrow \mathcal{B}(V)$ of $\odot(V, \mathcal{B}(V))$. As for the first condition, $\left.D_{-1}^{2}\right|_{\uparrow V}$ is obviously trivial while $\left.D_{-1}^{2}\right|_{\uparrow \mathcal{B}(V)}$ $=\uparrow d_{\mathcal{B}}^{2} \downarrow=0$.

The second equation is obviously trivial on $\uparrow V$ while on $\uparrow \mathcal{B}(V)$ we obtain

$$
\begin{aligned}
& D_{-1} D_{0}+D_{0} D_{-1}=D_{-1}(\uparrow \odot \uparrow)(\lambda+\rho)(\downarrow)+D_{0} \uparrow d_{\mathcal{B}} \downarrow=0 \\
& \quad=-\left(\uparrow \odot\left(\uparrow d_{\mathcal{B}}\right)\right)(\lambda)(\downarrow)+\left(\left(\uparrow d_{\mathcal{B}}\right) \odot \uparrow\right)(\rho)(\downarrow)+(\uparrow \odot \uparrow)(\lambda+\rho)\left(d_{\mathcal{B}}\right)(\downarrow) \\
& \quad=(\uparrow \odot \uparrow)\left[-\left(\mathbb{1} \odot d_{\mathcal{B}}\right)(\lambda)-\left(d_{\mathcal{B}} \odot \mathbb{1}\right)(\rho)+(\lambda+\rho)\left(d_{\mathcal{B}}\right)\right](\downarrow)
\end{aligned}
$$

which follows from (6). The sign introduced in the second line comes from moving $D_{-1}$ past $\uparrow$ and the sign in the third line comes from moving $\uparrow$ past $d_{\mathcal{B}}$.

Let us discuss the condition $D_{0}^{2}=0$. On $\uparrow V$ we have

$$
\begin{aligned}
D_{0}^{2} & =D_{0}(\uparrow \odot \uparrow)(\Delta)(\downarrow)=[((\uparrow \odot \uparrow)(\Delta) \odot \uparrow)-(\uparrow \odot(\uparrow \odot \uparrow)(\Delta))](\Delta)(\downarrow) \\
& =(\uparrow \odot \uparrow \odot \uparrow)[(\Delta \odot \mathbb{1}) \Delta-(\mathbb{1} \odot \Delta) \Delta](\downarrow) .
\end{aligned}
$$

Notice that, for $v \in V,(\Delta \odot \mathbb{1}) \Delta(v) \in \bigodot^{3}(V)$ is represented by $(\Delta \otimes \mathbb{1}) \Delta(v) \in V^{\otimes 3}$, while $(\mathbb{1} \odot \Delta) \Delta(v) \in \bigodot^{3}(V)$ is represented by $(\mathbb{1} \otimes \Delta) \Delta(v) \in V^{\otimes 3}$. The condition 
$(\Delta \odot \mathbb{1}) \Delta(v)=(\mathbb{1} \odot \Delta) \Delta(v)$ implies that $\left.D_{0}^{2}\right|_{\uparrow V}=0$ just as it implies $d_{C}^{2}=0$. On $\uparrow \mathcal{B}(V)$ we have

$D_{0}^{2}=(\uparrow \odot \uparrow \odot \uparrow)[(\Delta \odot \mathbb{1}) \lambda-(\mathbb{1} \odot \lambda) \lambda-(\mathbb{1} \odot \rho) \lambda+(\lambda \odot \mathbb{1}) \rho+(\rho \odot \mathbb{1}) \rho-(\mathbb{1} \odot \Delta) \rho](\downarrow)$ which is equivalent to the following three equations

$$
(\Delta \odot \mathbb{1}) \lambda=(\mathbb{1} \odot \lambda) \lambda,(\lambda \odot \mathbb{1}) \rho=(\mathbb{1} \odot \rho) \lambda \text { and }(\mathbb{1} \odot \Delta) \rho=(\rho \odot \mathbb{1}) \rho
$$

which are obvious analogs of the last three conditions from the definition of a bicomodule. These conditions may be violated already on $\mathcal{B}_{0}(V)$ as we explain after the next definition.

Lemma 3.2 suggests the possibility of interpreting the derivation $D_{0}$ as an infinitesimal deformation of $D_{-1}$ and we may try to integrate this infinitesimal deformation. The formal definition is the following.

Definition 3.3. By a homotopy $(V, \Delta)$-bicomodule structure on $\left(\mathcal{B}(V), d_{\mathcal{B}}\right)$ we mean a sequence $\left\{D_{k} \in \operatorname{Der}_{V}^{1}(\odot(V, \mathcal{B}(V))) ; k \geq 1\right\}$ such that (relative to the first bigrading)

$$
D_{k}\left(\bigodot^{i, j}(V, \mathcal{B}(V))\right) \subset \bigodot^{i+k+1, j}(V, \mathcal{B}(V))
$$

for $i, j \geq 0, k \geq 1$, and that $D:=D_{-1}+D_{0}+\sum_{k \geq 1} D_{k}$ has square zero, $D^{2}=0$.

One of the central results of the paper (Corollary 4.6) is that such a homotopy bicomodule structure exists.

We now come back to the failure of $\mathcal{B}_{0}(V)$ to satisfy the comodule compatibility conditions. Take for example the first equation of (8). Using generalized Sweedler notation, we set

$$
(\Delta \otimes \mathbb{1}) \Delta(a)=\sum a_{(1)}^{\prime} \otimes a_{(2)}^{\prime} \otimes a_{(3)}^{\prime}, \quad(\mathbb{1} \otimes \Delta) \Delta(a)=\sum a_{(1)}^{\prime \prime} \otimes a_{(2)}^{\prime \prime} \otimes a_{(3)}^{\prime \prime} .
$$

For $(a \mid b) \in V \otimes V=\mathcal{B}_{0}(V)$ we get

$$
\begin{gathered}
{[(\Delta \odot \mathbb{1}) \lambda-(\mathbb{1} \odot \lambda) \lambda](a \mid b)} \\
=\sum\left\{\left(a_{(1)}^{\prime} b_{(1)}^{\prime} \odot a_{(2)}^{\prime} b_{(2)}^{\prime}\right) \odot\left(a_{(3)}^{\prime} \mid b_{(3)}^{\prime}\right)-a_{(1)}^{\prime \prime} b_{(1)}^{\prime \prime} \odot\left(a_{(2)}^{\prime \prime} b_{(2)}^{\prime \prime} \odot\left(a_{(3)}^{\prime \prime} \mid b_{(3)}^{\prime \prime}\right)\right)\right\} \\
=\Phi^{-1} \bullet \sum\left\{\left(\Phi_{1} a_{(1)}^{\prime} b_{(1)}^{\prime} \odot \Phi_{2} a_{(2)}^{\prime} b_{(2)}^{\prime}\right) \odot\left(\Phi_{3} a_{(3)}^{\prime} \mid b_{(3)}^{\prime}\right)\right. \\
\left.-\left(a_{(1)}^{\prime \prime} b_{(1)}^{\prime \prime} \Phi_{1} \odot a_{(2)}^{\prime \prime} b_{(2)}^{\prime \prime} \Phi_{2}\right) \odot\left(a_{(3)}^{\prime \prime} \mid b_{(3)}^{\prime \prime} \Phi_{3}\right)\right\} \\
=\Phi^{-1} \bullet \sum\left\{\left(a_{(1)}^{\prime \prime} \Phi_{1} b_{(1)}^{\prime} \odot a_{(2)}^{\prime \prime} \Phi_{2} b_{(2)}^{\prime}\right) \odot\left(a_{(3)}^{\prime \prime} \Phi_{3} \mid b_{(3)}^{\prime}\right)\right. \\
\left.-\left(a_{(1)}^{\prime \prime} \Phi_{1} b_{(1)}^{\prime} \odot a_{(2)}^{\prime \prime} \Phi_{2} b_{(2)}^{\prime}\right) \odot\left(a_{(3)}^{\prime \prime} \mid \Phi_{3} b_{(3)}^{\prime}\right)\right\} \\
=\Phi^{-1} \bullet \sum\left\{\left(a_{(1)}^{\prime \prime} \Phi_{1} b_{(1)}^{\prime} \odot a_{(2)}^{\prime \prime} \Phi_{2} b_{(2)}^{\prime}\right) \odot\left[\left(a_{(3)}^{\prime \prime} \Phi_{3} \mid b_{(3)}^{\prime}\right)-\left(a_{(3)}^{\prime \prime} \mid \Phi_{3} b_{(3)}^{\prime}\right)\right]\right\} .
\end{gathered}
$$

The expression $\left(a_{(3)}^{\prime \prime} \Phi_{3} \mid b_{(3)}^{\prime}\right)-\left(a_{(3)}^{\prime \prime} \mid \Phi_{3} b_{(3)}^{\prime}\right)$ inside the right brackets in the last line is exactly $D_{-1}\left(a_{(3)}^{\prime \prime}\left|\Phi_{3}\right| b_{(3)}^{\prime}\right)$, therefore the deviation is, up to the overall factor, $\Phi^{-1}$ equal to $\left(\mathbb{1} \odot \mathbb{1} \odot D_{-1}\right)$ acting on

$$
\sum\left(a_{(1)}^{\prime \prime} \Phi_{1} b_{(1)}^{\prime} \odot a_{(2)}^{\prime \prime} \Phi_{2} b_{(2)}^{\prime}\right) \odot\left(a_{(3)}^{\prime \prime}\left|\Phi_{3}\right| b_{(3)}^{\prime}\right) .
$$

This suggests the form for the operator $D_{1}$. Consider the diagram for the Stasheff associahedron $K_{3}$ (see the next section for the definition)

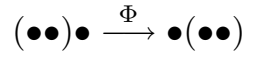


and the comultiplications associated to the two vertices

$$
\Delta^{(\bullet \bullet) \bullet}(a)=\sum a_{(1)}^{\prime} \otimes a_{(2)}^{\prime} \otimes a_{(3)}^{\prime}, \quad \Delta^{\bullet(\bullet \bullet)}(a)=\sum a_{(1)}^{\prime \prime} \otimes a_{(2)}^{\prime \prime} \otimes a_{(3)}^{\prime \prime} .
$$

Then (9) is a kind of product of three terms

$$
\Delta^{\bullet(\bullet \bullet)}(a), \quad \Phi, \quad \Delta^{(\bullet \bullet) \bullet}(b)
$$

corresponding to (10) but reversing the order because in the correspondence between functional notation and arrow notation,

$$
a \stackrel{T}{\longrightarrow} b \text { becomes } b=T(a) .
$$

All the operators $D_{n-2}$ will correspond to the geometry of the associahedron $K_{n}$ in precisely this way. We shall define a simplicial decomposition of $K_{n}$ having the same set of vertices. Then any $k$-simplex $\sigma=\left[v_{0}, v_{1}, \ldots, v_{k}\right]$ will be spanned by $k+1$ of the vertices of $K_{n}$ and we define an operator $M_{n}[\sigma]$ using the higher order comultiplications corresponding to the vertices and coassociativity operators corresponding to the edges. The details will be given in the next two sections.

\section{Some Properties of the StashefF ASsociahedra}

Let $K_{n}$ be, for $n \geq 2$, the Stasheff associahedron [16]. It is an (n-2)-dimensional cellular complex whose $i$-dimensional cells are indexed by the set $\operatorname{Br}_{n}(i)$ of all (meaningful) insertions of $(n-i-2)$ pairs of brackets between $n$ symbols, with suitably defined incidence maps. There is, for any $a, b \geq 2,0 \leq i \leq a-2,0 \leq j \leq$ $b-2$ and $1 \leq t \leq b$, a map

$$
(-,-)_{t}: \operatorname{Br}_{a}(i) \times \operatorname{Br}_{b}(j) \rightarrow \operatorname{Br}_{a+b-1}(i+j), u \times v \mapsto(u, v)_{t},
$$

where $(u, v)_{t}$ is given by the insertion of $(u)$ at the $t$-th place in $v$. This map defines, for $a, b \geq 2$ and $1 \leq t \leq b$, the inclusions $\iota_{t}: K_{a} \times K_{b} \hookrightarrow \partial K_{a+b-1}$ and it is known that

$$
\partial K_{n}=\bigcup_{J} \iota_{t}\left(K_{a} \times K_{b}\right),
$$

where $J=\{(a, b, t) ; a+b=n+1, a, b \geq 2$ and $1 \leq t \leq b\}$.

Let $\prec$ be the partial order on the set $\operatorname{Br}_{n}:=\mathrm{Br}_{n}(0)$ of the vertices of $K_{n}$ defined by saying that $u \prec v$ if and only if $v$ is obtained from $u$ by the substitution $\left(w_{1}, w_{2}\right) w_{3} \mapsto w_{1}\left(w_{2}, w_{3}\right)$ with some $w_{i} \in \operatorname{Br}_{n_{i}}, i=1,2,3, n_{1}+n_{2}+n_{3} \leq n$. Let $\xi_{n}$ be the (unique) minimal element of $\mathrm{Br}_{n}$. In the $\bullet$ notation, $\xi_{n}$ has parentheses expanding out from the left. For example $\xi_{4}=((\bullet \bullet) \bullet) \bullet$.

In the following proposition, instead of constructing $K_{n}$ as a cone over the full boundary $\partial K_{n}$, we use a cone from the minimal vertex $\xi_{n}$ to the part of the boundary consisting of faces not containing $\xi_{n}$. The proof, as well as that of Proposition 4.2 , both of which we omit, is of a purely combinatorial nature, using a simple induction.

Proposition 4.1. Let $\xi_{n}$ be the (unique) minimal element of $\mathrm{Br}_{n}$. Then

$$
K_{n}=\operatorname{Cone}\left(\bigcup_{J^{\prime}} \iota_{t}\left(K_{a} \times K_{b}\right) ; \xi_{n}\right),
$$

where $J^{\prime}=\{(a, b, t) \in J ; t \geq 2\}$.

The cone structure above induces on $K_{n}$ inductively the triangulation $\mathcal{T}_{n}$ as follows:

i. $\mathcal{T}_{2}$ is the only possible triangulation of $K_{2}=\{\bullet \bullet\}$ and 


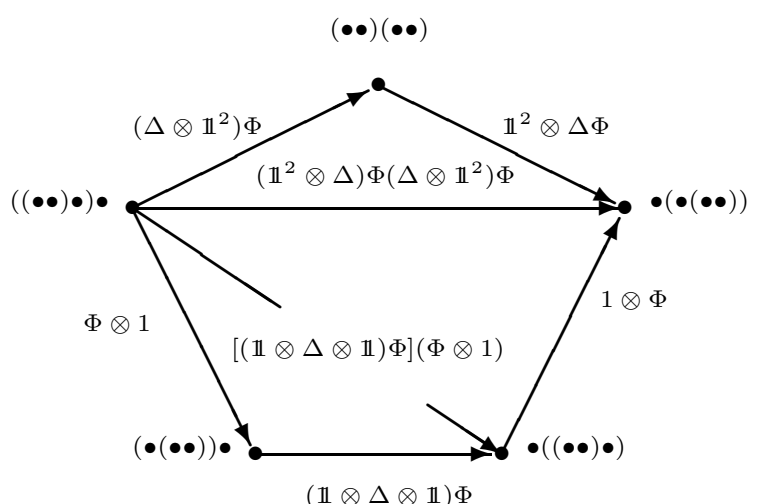

Figure 7. The distinguished triangulation of $K_{4}$.

ii. for $n \geq 2, \mathcal{T}_{n}$ is induced by the cone structure of Proposition 4.1 from the triangulations $\mathcal{T}_{a} \times \mathcal{T}_{b}$ of $K_{a} \times K_{b}, a+b=n+1, a, b \geq 2$, which are already defined, by the induction assumption.

Proposition 4.2. The triangulation $\mathcal{T}_{n}$ has the property that $\prec$ induces a total order on the vertices of any simplex of $\mathcal{T}_{n}$.

We will consider $\mathcal{T}_{n}$ as an oriented triangulation with the orientation induced by $\prec$. It will also be necessary to consider the reversed orientation which we will denote $\tilde{\mathcal{T}}_{n}$. Figure 7 shows the triangulation $\mathcal{T}_{n}$ for the pentagon $K_{4}$. This figure shows also the associativity operators which we introduce later.

The following definition is a differential and bigraded analog of the "pre-Lie system" introduced in [6] (compare also the notion of a nonunitary differential operad of [11]).

Definition 4.3. By a (right) differential comp algebra we mean a bigraded differential space $\left(X_{*, *}, d_{X}\right), d_{X}\left(X_{*, *}\right) \subset X_{*-1, *}$, together with a system of bilinear operations

$$
\diamond_{t}: X_{p, a} \otimes X_{q, b} \rightarrow X_{p+q, a+b-1}
$$

given for any $1 \leq t \leq b$ such that, for $\mathbf{s} \in X_{p, a}, \mathbf{t} \in X_{q, b}$ and $\mathbf{u} \in X_{r, c}$,

$$
\mathbf{s} \diamond_{i}\left(\mathbf{t} \diamond_{j} \mathbf{u}\right)= \begin{cases}(-1)^{p \cdot q} \cdot \mathbf{t} \diamond_{j+a-1}\left(\mathbf{s} \diamond_{i} \mathbf{u}\right), & \text { for } 1 \leq i \leq j-1, \\ \left(\mathbf{s} \diamond_{i-j+1} \mathbf{t}\right) \diamond_{j} \mathbf{u}, & \text { for } j \leq i \leq b+j-1, \text { and } \\ (-1)^{p \cdot q} \cdot \mathbf{t} \diamond_{j}\left(\mathbf{s} \diamond_{i-b+1} \mathbf{u}\right), & \text { for } i \geq j+b,\end{cases}
$$

and, for any $\mathbf{s} \in X_{p, a}$, and $\mathbf{t} \in X_{q, b}, 1 \leq t \leq b$,

$$
d_{X}\left(\mathbf{s} \diamond_{t} \mathbf{t}\right)=d_{X}(\mathbf{s}) \diamond_{t} \mathbf{t}+(-1)^{p} \cdot \mathbf{s} \diamond_{t} d_{X}(\mathbf{t}) .
$$

Let $C_{i}\left(K_{n}\right)$ denote the set of $i$-dimensional oriented simplicial chains of $\mathcal{T}_{n}$ with coefficients in $\mathbf{k}$ and let $d_{S}: C_{i}\left(K_{n}\right) \rightarrow C_{i-1}\left(K_{n}\right)$ be the simplicial differential. For $\mathbf{s} \in C_{p}\left(K_{a}\right)$ and $\mathbf{t} \in C_{q}\left(K_{b}\right), p, q \geq 0, a, b \geq 2$ and $1 \leq t \leq b$, let $\mathbf{s} \times \mathbf{t} \in$ $C_{p+q}\left(K_{a} \times K_{b}\right)$ denote the simplicial homology cross product (see [9, VIII.8]) and put

$$
\mathbf{s} \diamond_{t} \mathbf{t}:=\left(\iota_{t}\right)_{*}(\mathbf{s} \times \mathbf{t}) \in C_{p+q}\left(K_{a+b-1}\right) .
$$


Proposition 4.4. The simplicial chain complex $\left(C_{*}\left(K_{*}\right), d_{S}\right)$ together with operations $\diamond_{i}$ introduced above is a differential comp algebra in the sense of Definition 4.3.

Proof. Elementary combinatorial arguments give that, for $\alpha \in \mathrm{Br}_{a}(p), \beta \in \mathrm{Br}_{b}(q)$ and $\gamma \in \operatorname{Br}_{c}(r)$,

$$
\left(\alpha,(\beta, \gamma)_{j}\right)_{i}= \begin{cases}\left(\beta,(\alpha, \gamma)_{i}\right)_{j+a-1}, & \text { for } 1 \leq i \leq j-1, \\ \left((\alpha, \beta)_{i-j+1}, \gamma\right)_{j}, & \text { for } j \leq i \leq b+j-1, \text { and } \\ \left(\beta,(\alpha, \gamma)_{i-b+1}\right)_{j}, & \text { for } i \geq j+b .\end{cases}
$$

This implies that, for $x \in K_{a}, y \in K_{b}$ and $z \in K_{c}$,

$$
\iota_{i}\left(x \times \iota_{j}(y \times z)\right)= \begin{cases}\iota_{j+a-1}\left(y \times \iota_{i}(x \times z)\right), & \text { for } 1 \leq i \leq j-1, \\ \iota_{j}\left(\iota_{i-j+1}(x \times y) \times z\right), & \text { for } j \leq i \leq b+j-1, \text { and } \\ \iota_{j}\left(y \times \iota_{i-b+1}(x \times z)\right), & \text { for } i \geq j+b .\end{cases}
$$

Let $1 \leq i \leq j-1, \mathbf{s} \in C_{p}\left(K_{a}\right), \mathbf{t} \in C_{q}\left(K_{b}\right)$ and $\mathbf{u} \in C_{r}\left(K_{c}\right)$. Then, by definition,

$$
\begin{aligned}
\mathbf{s} \diamond_{i}\left(\mathbf{t} \diamond_{j} \mathbf{u}\right) & =\iota_{i *}\left(\mathbf{s} \times \iota_{j_{*}}(\mathbf{t} \times \mathbf{u})\right) \\
& =\left[\iota_{i}\left(\mathbb{1} \times \iota_{j}\right)\right]_{*}(\mathbf{s} \times \mathbf{t} \times \mathbf{u})=\left[\iota_{j+a-1}\left(\mathbb{1} \times \iota_{i}\right)\left(S_{21} \times \mathbb{1}\right)\right]_{*}(\mathbf{s} \times \mathbf{t} \times \mathbf{u}) \\
& =(-1)^{p q} \cdot\left[\iota_{j+a-1}\left(\mathbb{1} \times \iota_{i}\right)\right]_{*}(\mathbf{t} \times \mathbf{s} \times \mathbf{u})=(-1)^{p q} \cdot \mathbf{t} \diamond_{j+a-1}\left(\mathbf{s} \diamond_{i} \mathbf{u}\right),
\end{aligned}
$$

which proves the first case of (12). Here $S_{21}$ denotes the transposition of factors in the cartesian product, $S_{21}(x \times y)=y \times x$, and we used the associativity and (graded) commutativity property of the cross product, see [9]. The remaining cases can be discussed similarly. As for the condition (13), we have

$$
\begin{aligned}
d_{S}\left(\mathbf{s} \diamond_{t} \mathbf{t}\right) & =d_{S} \iota_{t *}(\mathbf{s} \times \mathbf{t})=\iota_{t *}\left(d_{S}(\mathbf{s} \times \mathbf{t})\right) \\
& =\iota_{t *}\left(d_{S}(\mathbf{s}) \times \mathbf{t}+(-1)^{p} \cdot \mathbf{s} \times d_{S}(\mathbf{t})\right)=d_{S}(\mathbf{s}) \diamond_{t} \mathbf{t}+(-1)^{p} \cdot \mathbf{s} \diamond_{t} d_{S}(\mathbf{t}) .
\end{aligned}
$$

Here we used the derivation property of the cross product [9].

We have also the following analog of [6, Theorem 2].

Proposition 4.5. Let $\left(X_{*, *}, d_{X}, \diamond_{*}\right)$ be a differential comp algebra as in Definition 4.3. Put, for $\mathbf{s} \in X_{p, a}$ and $\mathbf{t} \in X_{q, b}$,

$$
\mathbf{s} \diamond \mathbf{t}:=\sum_{1 \leq t \leq b}(-1)^{(a+1)(t+q+1)} \cdot \mathbf{s} \diamond_{t} \mathbf{t} .
$$

Then $\diamond$ induces on $X^{*}:=\bigoplus_{a-p-1=*} X_{p, a}$ the structure of a differential graded preLie algebra (compare the nondifferential analog of this construction in [6, par. 2], that is

$$
\begin{aligned}
\mathbf{s} \diamond(\mathbf{t} \diamond \mathbf{u})-(\mathbf{s} \diamond \mathbf{t}) \diamond \mathbf{u} & =(-1)^{|\mathbf{s}| \cdot|\mathbf{t}|}(\mathbf{t} \diamond(\mathbf{s} \diamond \mathbf{u})-(\mathbf{t} \diamond \mathbf{s}) \diamond \mathbf{u}) \text { and } \\
d_{X}(\mathbf{s} \diamond \mathbf{t}) & =d_{X} \mathbf{s} \diamond \mathbf{t}+(-1)^{|\mathbf{s}|} \cdot \mathbf{s} \diamond d_{X} \mathbf{t}
\end{aligned}
$$

for any $\mathbf{s} \in X^{|\mathbf{s}|}, \mathbf{t} \in X^{|\mathbf{t}|}$ and $\mathbf{u} \in X^{|\mathbf{u}|}$. Consequently, the formula

$$
[\mathbf{s}, \mathbf{t}]:=\mathbf{s} \diamond \mathbf{t}-(-1)^{|\mathbf{s}| \cdot|\mathbf{t}|} \mathbf{t} \diamond \mathbf{s}
$$

defines on $\left(X^{*}, d_{X}\right)$ the structure of a differential graded Lie algebra.

Proof. A straightforward verification.

We have the obvious corollary of Proposition 4.4 and Proposition 4.5.

Corollary 4.6. The comp structure of (14) induces on $C(K)^{*}:=\bigoplus_{n-p-1=*} C_{p}\left(K_{n}\right)$ the structure of a differential graded Lie algebra $\left(C(K)^{*},[-,-], d_{S}\right)$. 
We end this section with the following proposition:

Proposition 4.7. There exists a sequence $\mathbf{e}_{n} \in C_{n}\left(K_{n+2}\right) \subset C(K)^{1}, n \geq 0$, of "fundamental classes" such that

$$
\mathbf{e}_{0}=1 \cdot(\bullet \bullet) \text { and } d_{S}\left(\mathbf{e}_{n}\right)+\frac{1}{2} \sum_{i+j=n-1}\left[\mathbf{e}_{i}, \mathbf{e}_{j}\right]=0 .
$$

Proof. The proof is based on the acyclicity of the associahedra (see $[15,16])$. Suppose inductively that $\mathbf{e}_{k}$ have been constructed for any $k<n$. Then, since all the $\mathbf{e}_{i}$ have degree 1 ,

$$
\begin{aligned}
d_{S}\left(\frac{1}{2} \sum_{i+j=n-1}\left[\mathbf{e}_{i}, \mathbf{e}_{j}\right]\right) & =\frac{1}{2} \sum_{i+j=n-1}\left(\left[d_{S}\left(\mathbf{e}_{i}\right), \mathbf{e}_{j}\right]-\left[\mathbf{e}_{i}, d_{S}\left(\mathbf{e}_{j}\right)\right]\right) \\
& =-\frac{1}{4} \sum_{\alpha+\beta+\gamma=n-2}\left(\left[\left[\mathbf{e}_{\alpha}, \mathbf{e}_{\beta}\right], \mathbf{e}_{\gamma}\right]-\left[\mathbf{e}_{\gamma},\left[\mathbf{e}_{\alpha}, \mathbf{e}_{\beta}\right]\right]\right) \\
& =\frac{1}{2} \sum_{\alpha+\beta+\gamma=n-2}\left[\mathbf{e}_{\gamma},\left[\mathbf{e}_{\alpha}, \mathbf{e}_{\beta}\right]\right]=0
\end{aligned}
$$

by the Jacobi identity. Therefore $\frac{1}{2} \sum_{i+j=n-1}\left[\mathbf{e}_{i}, \mathbf{e}_{j}\right]$ is a cycle in $C_{n-1}\left(K_{n+2}\right)$ and the existence of $\mathbf{e}_{n}$ follows from the acyclicity of $K_{n+2}$.

Let us give an explicit description of the $\diamond_{i}$-product. By an $(r, s)$-shuffle we mean, for $r, s \geq 1$, a partition of the set $\{1, \ldots, r+s\}$ into two disjoint subsets $\left\{i_{1}<\cdots<i_{r}\right\}$ and $\left\{j_{1}<\cdots<j_{s}\right\}$. Denote by $\operatorname{Sh}_{r, s}$ the set of all $(r, s)$-shuffles. For $\phi \in \operatorname{Sh}_{r, s}$, let $\operatorname{sgn}(\phi)$ denote the signum of the permutation $(1, \ldots, r+s) \mapsto$ $\left(i_{1}, \ldots, i_{r}, j_{1}, \ldots, j_{s}\right)$. Any $\phi \in \mathrm{Sh}_{r, s}$ defines a sequence $\left\{\left(\phi^{\prime}(l), \phi^{\prime \prime}(l)\right) ; 0 \leq l \leq\right.$ $r+s\} \subset(0, \ldots, r) \times(0, \ldots, s)$ by

$$
\phi^{\prime}(l):=\operatorname{Card}\left\{k ; i_{k} \leq l\right\} \text { and } \phi^{\prime \prime}(l):=\operatorname{Card}\left\{k ; j_{k} \leq l\right\}, 0 \leq l \leq r+s .
$$

For $\mathbf{s}=\left[v_{0}, \ldots, v_{r}\right] \in c_{r}\left(K_{a}\right)$ and $\mathbf{t}=\left[w_{0}, \ldots, w_{r}\right] \in C_{s}\left(K_{b}\right)$, the simplicial cross product is given by

$$
\mathbf{s} \times \mathbf{t}:=\sum_{\phi \in \operatorname{Sh}_{r, s}} \operatorname{sgn}(\phi)\left[\left(v_{\phi^{\prime}(0)}, w_{\phi^{\prime \prime}(0)}\right), \ldots,\left(v_{\phi^{\prime}(r+s)}, w_{\phi^{\prime \prime}(r+s)}\right)\right] \in C_{r+s}\left(K_{a} \times K_{b}\right),
$$

see [9]. This gives the formula

$$
\begin{array}{r}
\mathbf{s} \diamond_{t} \mathbf{t}=\sum_{\phi \in \mathrm{Sh}_{r, s}} \operatorname{sgn}(\phi)\left[\left(v_{\phi^{\prime}(0)}, w_{\phi^{\prime \prime}(0)}\right)_{t}, \ldots,\left(v_{\phi^{\prime}(r+s)}, w_{\phi^{\prime \prime}(r+s)}\right)_{t}\right] \in C_{r+s}\left(K_{a+b-1}\right), \\
1 \leq t \leq b .
\end{array}
$$

Before concluding this section we would like to note a further analogy between $C(K)^{*}$ and the algebraic structures investigated by Gerstenhaber in [6]. There is a second product $*$ on $\uparrow C(K)^{*}$ which is compatible with the $\diamond$-product in the sense that, for any $\rho \in C_{r}\left(K_{a}\right)$, the operation $\rho \diamond(-)$ is a $*$-derivation of degree $a-r-1$.

To begin with we have an imbedding

$$
\phi: K_{a} \times K_{b} \rightarrow K_{a+b}
$$

given by concatenation of bracketings, $\phi(u, v):=(u)(v)$. For $\sigma \in C_{s}\left(K_{b}\right)$ and $\tau \in C_{t}\left(K_{c}\right)$ define

$$
\sigma * \tau:=(-1)^{b t} \phi_{*}(\sigma \times \tau) \in C_{s+t}\left(K_{b+c}\right),
$$


where $\times$ is the simplicial homology cross product. Since $\phi(\phi \times \mathbb{1}) \neq \phi(\mathbb{1} \times \phi)$, this product is not associative. Nor is it graded commutative. A straightforward calculation shows that, for $\rho \in C_{r}\left(K_{a}\right)$,

$$
\rho \diamond(\sigma * \tau)=(\rho \diamond \sigma) * \tau+(-1)^{(a-r-1)(s+b)} \sigma *(\rho \diamond \tau) .
$$

Furthermore,

$$
d_{S}(\sigma * \tau)=\left(d_{S} \sigma\right) * \tau+(-1)^{s+b} \sigma *\left(d_{S} \tau\right)
$$

What we have just described is presented for its intrinsic interest. We do not as yet have an application for this product in deformation theory.

\section{MAIN THEOREM}

Let $D_{-1}, D_{0} \in \operatorname{Der}_{V}^{1}(\odot(V, \mathcal{B}(V)))$ be as in Lemma 3.2. Observe that $\operatorname{Der}_{V}^{*}(\odot(V, \mathcal{B}(V)))$ is a differential graded Lie algebra, the bracket being given by the (graded) commutator of derivations and the differential defined by $\nabla(\theta):=$ $\left[D_{-1}, \theta\right]$. Let $\left(C(K)^{*},[-,-], d_{S}\right)$ be the graded Lie algebra from Corollary 4.6.

Theorem 5.1. There exists a homomorphism

$$
m:\left(C(K)^{*},[-,-], d_{S}\right) \rightarrow\left(\operatorname{Der}_{V}^{*}(\bigodot(V, \mathcal{B}(V))),[-,-], \nabla\right)
$$

of differential graded Lie algebras such that $m\left(\mathbf{e}_{0}\right)=D_{0}$. The homomorphism $m$ has the properties that

$$
\begin{aligned}
& m\left(C_{k}\left(K_{n}\right)\right)\left(\bigodot(V, \mathcal{B}(V))^{i, j}\right) \subset \bigodot(V, \mathcal{B}(V))^{i+n-1, j-k}, \\
& m\left(C_{k}\left(K_{n}\right)\right)\left(\bigodot^{i, j}(V, \mathcal{B}(V))\right) \subset \bigodot^{i+n-1, j}(V, \mathcal{B}(V)),
\end{aligned}
$$

for $i, j, k \geq 0, n \geq 2$.

As a corollary, we get the existence of a homotopy $(V, \Delta)$-bicomodule structure on the bar resolution $\left(\mathcal{B}(V), d_{\mathcal{B}}\right)$.

Corollary 5.2. The derivations $D_{k}:=m\left(\mathbf{e}_{k}\right), k \geq 1$, define a homotopy $(V, \Delta)$ bicomodule structure on the bar resolution $\left(\mathcal{B}(V), d_{\mathcal{B}}\right)$.

Proof. Let $D=D_{-1}+D_{0}+\sum_{k \geq 1} D_{k}$. The only thing which has to be verified is the condition $D^{2}=0$. Looking at the homogeneous components we see that $D^{2}=0$ is equivalent to

$$
\left[D_{-1}, D_{k}\right]+\frac{1}{2} \sum_{i+j=k-1}\left[D_{i}, D_{j}\right]=0
$$

which is, by the definition of the derivations $D_{k}$ and Theorem 5.1, the same as

$$
m\left(d_{S}\left(\mathbf{e}_{k}\right)+\frac{1}{2} \sum_{i+j=k-1}\left[\mathbf{e}_{i}, \mathbf{e}_{j}\right]\right)=0,
$$

which follows from (15).

As in [13], define inductively, for any $u \in \mathrm{Br}_{n}$, the iterated comultiplication $\Delta^{\{u\}}: V \rightarrow V^{\otimes n}$ by

$$
\Delta^{\{\bullet\}}=\mathbb{1}, \Delta^{\{\bullet \bullet\}}=\Delta \text { and } \Delta^{\left\{(u, v)_{t}\right\}}=\left(\mathbb{1}^{\otimes(t-1)} \otimes \Delta^{\{u\}} \otimes \mathbb{1}^{\otimes(n-t)}\right) \Delta^{\{v\}},
$$


$u \in \mathrm{Br}_{m}, v \in \mathrm{Br}_{n}$ and $1 \leq t \leq n$. For example, $\Delta^{\{\bullet(\bullet \bullet)\}}=(\mathbb{1} \otimes \Delta) \Delta, \Delta\{(\bullet \bullet)(\bullet \bullet)\}=$ $(\Delta \otimes \Delta) \Delta$, etc. It is also a good exercise to prove that, for $x \in \mathrm{Br}_{a}, y \in \mathrm{Br}_{b}$ and $z \in \mathrm{Br}_{c}$,

$$
\begin{aligned}
& \Delta^{\{x(y z)\}}=\left(\Delta^{\{x\}} \otimes \Delta^{\{y\}} \otimes \Delta^{\{z\}}\right)(\mathbb{1} \otimes \Delta) \Delta \text { and } \\
& \Delta^{\{(x y) z\}}=\left(\Delta^{\{x\}} \otimes \Delta^{\{y\}} \otimes \Delta^{\{z\}}\right)(\Delta \otimes \mathbb{1}) \Delta .
\end{aligned}
$$

Sometimes we use the "generalized Sweedler notation", meaning that for $a \in V$ and $u \in \mathrm{Br}_{n}$ we write

$$
\Delta^{\{u\}}(a)=\sum a^{(u, 1)} \otimes \cdots \otimes a^{(u, n)}:=\sum \bigotimes_{i=1}^{n} a^{(u, n)} \in V^{\otimes n} .
$$

For any $u, v \in \mathrm{Br}_{n}$ consider the associativity operator $\Psi_{u, v} \in V^{\otimes n}$ having the following two properties.

i. $\Psi_{u, u}=1^{\otimes n}$ and $\Psi_{u, v} \cdot \Psi_{v, w}=\Psi_{u, w}$, for any $u, v, w \in \operatorname{Br}_{n}$ (the quasigroup property).

ii. Let $u=(x(y z), w)_{t}$ and $v=((x y) z, w)_{t}$, for some $x \in \mathrm{Br}_{a}, y \in \mathrm{Br}_{b}, z \in \mathrm{Br}_{c}$, $w \in \mathrm{Br}_{m}$, with $a+b+c+m=n+1$. In plain words this means that $v$ was obtained by the substitution $x(y z) \mapsto(x y) z$ at the $t$-th place in $u$. Then

$$
\Psi_{u, v}=1^{\otimes(t-1)} \otimes\left(\Delta^{\{x\}} \otimes \Delta^{\{y\}} \otimes \Delta^{\{z\}}\right)(\Phi) \otimes 1^{\otimes(m-t)} .
$$

These two conditions really define $\Psi_{u, v}$ for all $u, v \in \mathrm{Br}_{n}$. Indeed, for any $u, v \in \mathrm{Br}_{n}$ there exists a sequence of "elementary moves" from $u$ to $v$ as in (ii), and we may define $\Psi_{u, v}$ using the quasigroup property (i). The coherence then says that this definition does not depend on the particular choice of elementary moves. The following lemma gives a relation between the associativity operators and iterated comultiplications.

Lemma 5.3. For any $u, v \in \mathrm{Br}_{n}$ and $a \in V$,

$$
\Psi_{u, v} \cdot \Delta^{\{v\}}(a)=\Delta^{\{u\}}(a) \cdot \Psi_{u, v} .
$$

Using the notation of (17), we may rewrite (18) symbolically as

$$
\sum \bigotimes_{i=1}^{n} \Psi_{u, v}^{i} \cdot a^{(v, i)}=\sum \bigotimes_{i=1}^{n} a^{(u, i)} \cdot \Psi_{u, v}^{i},
$$

with $\Psi_{u, v}=\sum \Psi_{u, v}^{1} \otimes \cdots \otimes \Psi_{u, v}^{n}$.

Proof. First, let $u=x(y z)$ and $v=(x y) z$. Then we have, by the definition of the associativity operator and (16),

$$
\begin{aligned}
\Psi_{u, v} \cdot \Delta^{\{v\}}(a) & =\left(\Delta^{\{x\}} \otimes \Delta^{\{y\}} \otimes \Delta^{\{z\}}\right)(\Phi) \cdot\left(\Delta^{\{x\}} \otimes \Delta^{\{y\}} \otimes \Delta^{\{z\}}\right)(\Delta \otimes \mathbb{1}) \Delta(a) \\
& =\left(\Delta^{\{x\}} \otimes \Delta^{\{y\}} \otimes \Delta^{\{z\}}\right)[\Phi \cdot(\Delta \otimes \mathbb{1}) \Delta(a)] \\
& =\left(\Delta^{\{x\}} \otimes \Delta^{\{y\}} \otimes \Delta^{\{z\}}\right)[(\mathbb{1} \otimes \Delta) \Delta(a) \cdot \Phi] \\
& =\left(\Delta^{\{x\}} \otimes \Delta^{\{y\}} \otimes \Delta^{\{z\}}\right)(\mathbb{1} \otimes \Delta) \Delta(a) \cdot\left(\Delta^{\{x\}} \otimes \Delta^{\{y\}} \otimes \Delta^{\{z\}}\right)(\Phi) \\
& =\Delta^{\{u\}}(a) \Psi_{u, v} .
\end{aligned}
$$

From this we prove (18) easily for any "elementary move" as in (ii) above. The statement for a general couple $u, v$ then follows from the fact that there exists a sequence of elementary moves from $u$ to $v$ and the resulting operator $\Psi_{u, v}$ is independent of the choice of particular sequence. 
Let $u \in \mathrm{Br}_{n}$ and $x_{1}, \ldots, x_{n} \in \odot(N)$. Denote by $\bigodot_{u}\left(x_{1}, \ldots, x_{n}\right)$ the product of the elements $x_{1}, \ldots, x_{n}$ in the nonassociative algebra $\odot(N)$ with the bracketing $u$. For example, $\bigodot_{\bullet(\bullet \bullet)}\left(x_{1}, x_{2}, x_{3}\right)=x_{1} \odot\left(x_{2} \odot x_{3}\right)$, etc.

Let $x_{1}, \ldots, x_{n}$ be elements from $\odot(N)$ and let $\phi=\sum \phi_{1} \otimes \cdots \otimes \phi_{n} \in V^{\otimes n}$. By $\phi \bullet\left(x_{1}, \ldots, x_{n}\right)$ we denote the element $\sum\left(\phi_{1} \bullet x_{1}, \ldots, \phi_{n} \bullet x_{n}\right)$. In other words, we denote by the same symbol the left action of the algebra $(V, \cdot)$ on $\odot(N)$ and the induced action of $\left(V^{\otimes n}, \cdot\right)$ on $\bigodot^{n}(N)$. The same notation with $\phi$ on the right will be used also for the right action. The following lemma gives a characterization of the associativity operators up to a central element.

Lemma 5.4. Let $u, v \in \mathrm{Br}_{n}$ and $x_{1}, \ldots, x_{n} \in \bigodot(N)$. Then

$$
\bigodot_{u}\left(\Psi_{u, v} \bullet\left(x_{1}, \ldots, x_{n}\right)\right)=\bigodot_{v}\left(\left(x_{1}, \ldots, x_{n}\right) \bullet \Psi_{u, v}\right) .
$$

Proof. The lemma follows immediately from the definition of the associativity operator and the defining relation (3).

Notice that, for $u=\bullet(\bullet \bullet)$ and $v=(\bullet \bullet) \bullet, \Psi_{u, v}=\Phi$ and (20) says that

$$
\Phi \bullet\left[x_{1} \odot\left(x_{2} \odot x_{3}\right)\right]=\left[\left(x_{1} \odot x_{2}\right) \odot x_{2}\right] \bullet \Phi,
$$

which is exactly (3).

For $u, v \in \mathrm{Br}_{n}$ and $x_{1}, \ldots, x_{n} \in \odot(N)$, let

$$
\square_{u, v}\left(x_{1}, \ldots, x_{n}\right):=\bigodot_{u}\left(\Psi_{u, v} \bullet\left(x_{1}, \ldots, x_{n}\right)\right) .
$$

We have the following lemma.

Lemma 5.5. For $u, v, w \in \mathrm{Br}_{n}, x_{1}, \ldots, x_{n} \in \odot(N)$ and $a, b \in V$ we have

$$
\begin{aligned}
a \bullet \square_{u, v}\left(x_{n}, \ldots, x_{1}\right) & =\square_{u, v}\left(\Delta^{\{v\}}(a) \bullet\left(x_{1}, \ldots, x_{n}\right)\right), \\
\square_{u, v}\left(x_{n}, \ldots, x_{1}\right) \bullet b & =\square_{u, v}\left(\left(x_{1}, \ldots, x_{n}\right) \bullet \Delta^{\{u\}}(b)\right), \\
\square_{u, w}\left(\Psi_{w, v} \bullet\left(x_{1}, \ldots, x_{n}\right)\right) & =\square_{u, v}\left(x_{1}, \ldots, x_{n}\right) \text { and } \\
\square_{u, w}\left(\left(x_{1}, \ldots, x_{n}\right) \bullet \Psi_{v, u}\right) & =\square_{v, w}\left(x_{1}, \ldots, x_{n}\right) .
\end{aligned}
$$

Proof. The first two equations follow immediately from [13, Lemma 3.1]. As for the third one, we have

$$
\begin{aligned}
\square_{u, w}\left(\Psi_{w, v} \bullet\left(x_{1}, \ldots, x_{n}\right)\right) & =\bigodot_{u}\left(\Psi_{u, w} \bullet\left(\Psi_{w, v} \bullet\left(x_{1}, \ldots, x_{n}\right)\right)\right) \\
& =\bigodot_{u}\left(\left(\Psi_{u, w} \cdot \Psi_{w, v}\right) \bullet\left(x_{1}, \ldots, x_{n}\right)\right) \\
& =\bigodot_{u}\left(\Psi_{u, v} \bullet\left(x_{1}, \ldots, x_{n}\right)\right)=\square_{u, v}\left(x_{1}, \ldots, x_{n}\right) .
\end{aligned}
$$

Here we use that $\Psi_{u, w} \cdot \Psi_{w, v}=\Psi_{u, v}$ which follows from the quasigroup property of the associativity operator. The last equation can be proved similarly.

Lemma 5.6. Let $u, v \in \mathrm{Br}_{a}, z, w \in \mathrm{Br}_{b}, x_{1}, \ldots, x_{a}, y_{1}, \ldots, y_{b} \in \odot(N)$ and $1 \leq$ $t \leq b$. Then

$$
\begin{aligned}
& \square_{z, w}\left(y_{1}, \ldots, y_{t-1}, \square_{u, v}\left(x_{1}, \ldots, x_{a}\right), y_{t+1}, \ldots, y_{b}\right) \\
& \quad=\square_{(u, z)_{t},(v, w)_{t}}\left(y_{1}, \ldots, y_{t-1}, x_{1}, \ldots, x_{a}, y_{t+1}, \ldots, y_{b}\right) .
\end{aligned}
$$

Proof. An easy exercise on the definitions which we leave for the reader. 
The map $m$ of Theorem 5.1 will be defined using another map

$$
M[\sigma]: \mathcal{B}(V) \rightarrow \bigodot^{n-1,1}(V, \mathcal{B}(V)),
$$

given for any $\tilde{\mathcal{T}}_{n}$-oriented $k$-simplex $\sigma=\left[v_{0}, \ldots, v_{k}\right]$ of $K_{n}, n \geq 2, k \geq 1$ and $v_{0}, \ldots, v_{k} \in \mathrm{Br}_{n}, v_{k} \prec v_{k-1} \prec \cdots \prec v_{0}$ (recall that $\tilde{\mathcal{T}}_{n}$ is the orientation opposite to the one induced by the partial order of vertices of the associahedron $K_{n}$ ). For any such $\sigma, m(\sigma)$ will be a derivation given by

$$
\left.m(\sigma)\right|_{\uparrow V}:=0 \text { and }\left.m(\sigma)\right|_{\uparrow \mathcal{B}(V)}:=\left(\uparrow^{\odot n}\right) \circ M[\sigma] \circ \downarrow .
$$

Let $P(q, k)$ be, for $k \geq 0$ and $q \geq 1$, the set of all subdivisions of the segment of $(q+2)$ elements into $(k+1)$ subsegments such that the first and the last segment of the subdivision is nonempty. The elements of $P(q, k)$ can be encoded by nondecreasing maps $\pi:(1, \ldots, k) \rightarrow(1, \ldots, q+1)$. The corresponding subdivision will be then

$$
(0, \ldots, \pi(1)-1)(\pi(1), \ldots, \pi(2)-1) \cdots(\pi(k), \ldots, q+1) .
$$

Let $\hat{a}=a_{0} \otimes \cdots \otimes a_{q+1} \in \mathcal{B}_{-q}(V)$. For $0 \leq j \leq k$ write

$$
\pi_{j}(\hat{a}):=a_{\pi(j)} \otimes \cdots \otimes a_{\pi(j+1)-1} .
$$

Denote also, for $\pi \in P(q, k)$,

$$
o(\pi)=\sum_{i=1}^{k} \pi(i) .
$$

Let $\bar{\otimes}$ denote the multiplication in the tensor product of tensor algebras. In particular, for $x_{1}, \ldots, x_{m} \in(\otimes V)^{\otimes d}$ with $x_{j}=x_{j}^{1} \otimes \cdots \otimes x_{j}^{d}$ and $x_{j}^{i} \in \otimes V$ for $1 \leq j \leq m$, we have

$x_{1} \bar{\otimes} \cdots \bar{\otimes} x_{m}:=\left(x_{1}^{1} \otimes \cdots \otimes x_{m}^{1}\right) \otimes\left(x_{1}^{2} \otimes \cdots \otimes x_{m}^{2}\right) \otimes \cdots \otimes\left(x_{1}^{d} \otimes \cdots \otimes x_{m}^{d}\right) \in(\bigotimes V)^{\otimes d}$.

Using this notation, let $\Psi_{\pi}[\sigma](\hat{a}) \in \bigotimes^{n} V^{\otimes(q+k+2)}$ be defined, for any $\pi \in P(q, k)$, as

$$
\Psi_{\pi}[\sigma](\hat{a}):=\Delta^{\left\{v_{0}\right\}}\left(\pi_{0}(\hat{a})\right) \bar{\otimes} \Psi_{v_{0}, v_{1}} \bar{\otimes} \Delta^{\left\{v_{1}\right\}}\left(\pi_{1}(\hat{a})\right) \bar{\otimes} \cdots \bar{\otimes} \Psi_{v_{k-1}, v_{k}} \bar{\otimes} \Delta^{\left\{v_{k}\right\}}\left(\pi_{k}(\hat{a})\right) .
$$

Here we used the shorthand $\Delta^{\left\{v_{j}\right\}}\left(\pi_{j}(\hat{a})\right)$ for

$$
\Delta^{\left\{v_{j}\right\}}\left(a_{\pi(j)}\right) \bar{\otimes} \cdots \bar{\otimes} \Delta^{\left\{v_{j}\right\}}\left(a_{(\pi(j+1)-1)}\right) .
$$

Note that there is an order preserving bijection between the factors of this product and the elements (vertices and edges) of the directed graph determined by the one skeleton of $\sigma$.

Since the multiplication - on $V$ is associative, we can iterate it to a map $\bar{\mu}$ : $V^{\otimes m} \rightarrow V$, for any $m \geq 1$. Writing $\Psi_{\pi}[\sigma](\hat{a})$ as $\sum \phi_{1} \otimes \cdots \otimes \phi_{n}$, with $\phi_{i} \in$ $V^{\otimes(q+k+2)}, 1 \leq i \leq n$, let $\bar{\mu}_{r}\left(\Psi_{\pi}[\sigma](\hat{a})\right) \in V^{\otimes(r-1)} \otimes \mathcal{B}_{-q-k}(V) \otimes V^{\otimes(n-r)}$ be defined as

$$
\bar{\mu}_{r}\left(\Psi_{\pi}[\sigma](\hat{a})\right):=\sum \bar{\mu}\left(\phi_{1}\right) \otimes \cdots \otimes \bar{\mu}\left(\phi_{r-1}\right) \otimes \phi_{r} \otimes \bar{\mu}\left(\phi_{r+1}\right) \otimes \cdots \otimes \bar{\mu}\left(\phi_{n}\right) .
$$

For any $1 \leq r \leq n$, define

$$
M_{\pi}^{r}[\sigma](\hat{a}):=\square_{v_{k}, v_{0}}\left(\bar{\mu}_{r}\left(\Psi_{\pi}[\sigma](\hat{a})\right)\right) .
$$

Here, as usual, we identify the multilinear map $\square_{v_{k}, v_{0}}$ with a linear map on the tensor product. Finally we introduce a sign factor:

$$
\operatorname{sgn}(\pi, \sigma):=(-1)^{o(\pi)+k n},
$$


where $\sigma \in C_{k}\left(K_{n}\right)$, and define

$$
M[\sigma]:=\sum_{1 \leq r \leq n} M^{r}[\sigma](\hat{a}) \text { with } M^{r}[\sigma]:=\sum_{\pi \in P(q, k)} \operatorname{sgn}(\pi, \sigma) \cdot M_{\pi}^{r}[\sigma]
$$

Lemma 5.7. For any $k$-simplex $\sigma=\left[v_{0}, \ldots, v_{k}\right]$ of $K_{n}$

$$
m\left(d_{S} \sigma\right)=\nabla(m(\sigma)) .
$$

Proof. Let us express first the terms of (23) via the defining map $M[-]$. We have

$$
m\left(d_{S} \sigma\right)=\uparrow^{\odot n} M\left[d_{S} \sigma\right] \downarrow
$$

while

$$
\begin{aligned}
\nabla(m(\sigma))= & {\left[D_{-1} \circ m(\sigma)\right]+(-1)^{k+n} \cdot\left[m(\sigma) \circ D_{-1}\right] } \\
= & D_{-1}\left(\uparrow \odot^{n} M[\sigma]\right) \downarrow+(-1)^{k+n} \cdot m(\sigma)\left(\uparrow d_{\mathcal{B}} \downarrow\right) \\
= & \sum_{1 \leq r \leq n}(-1)^{n-1} \cdot\left(\uparrow \odot^{\odot n}\right)\left(\mathbb{1}^{\odot(r-1)} \odot d_{\mathcal{B}} \odot \mathbb{1}^{\odot(n-r)}\right) M^{r}[\sigma] \downarrow \\
& +(-1)^{k+n} \cdot(\uparrow \odot n) M[\sigma] d_{\mathcal{B}} \downarrow .
\end{aligned}
$$

The sign $(-1)^{n-1}$ is explained as follows:

$$
\begin{aligned}
D_{-1}(\uparrow \odot n & \left.M^{r}[\sigma]\right) \\
& =\sum_{1 \leq r \leq n}\left(\mathbb{1}^{\odot(r-1)} \odot \uparrow d_{\mathcal{B}} \downarrow \odot \mathbb{1}^{\odot(n-r)}\right)\left(\uparrow \odot^{n} M^{r}[\sigma]\right)=/ \text { transpose } \uparrow^{\odot(r-1)}, \uparrow d_{\mathcal{B}} \downarrow / \\
& =\sum_{1 \leq r \leq n}(-1)^{(r-1)} \cdot\left(\uparrow \odot(r-1) \odot \uparrow d_{\mathcal{B}} \odot \uparrow \odot(n-r)\right) M[\sigma]=/ \text { transpose } d_{\mathcal{B}}, \uparrow \odot(n-r) / \\
& =\sum_{1 \leq r \leq n}(-1)^{(r-1)+(n-r)} \cdot\left(\uparrow \odot(r-1) \odot \uparrow \odot \uparrow^{\odot(n-r)}\right)\left(\mathbb{1}^{\odot(r-1)} \odot d_{\mathcal{B}} \odot \mathbb{1}^{\odot(n-r)}\right) M[\sigma] \\
& =\sum_{1 \leq r \leq n}(-1)^{n-1} \cdot\left(\uparrow{ }^{\odot n}\right)\left(\mathbb{1}^{\odot(r-1)} \odot d_{\mathcal{B}} \odot \mathbb{1}^{\odot(n-r)}\right) M^{r}[\sigma]
\end{aligned}
$$

which gives the sign as claimed.

Looking at the component (in $\bigodot^{n-1,1}(V)$ ) having an element of $\mathcal{B}(V)$ at the $r$-th place we see that (23) is equivalent to

$$
\begin{aligned}
M^{r}\left[d_{S} \sigma\right](\hat{a})= & (-1)^{n-1} \cdot\left(\mathbb{1}^{\odot(r-1)} \odot d_{\mathcal{B}} \odot \mathbb{1}^{\odot(n-r)}\right) M^{r}[\sigma](\hat{a}) \\
& +(-1)^{n+k} \cdot M^{r}[\sigma]\left(d_{\mathcal{B}}(\hat{a})\right),
\end{aligned}
$$

which has to be satisfied for any $1 \leq r \leq n$.

Let us discuss the first term on the right-hand side of (24). Let $\hat{a} \in \mathcal{B}_{-q}(V)$ so that $M^{r}[\sigma](\hat{a}) \in \mathcal{B}_{-q-k}(V)$. The differential $d_{\mathcal{B}}$ applied on an element from $\mathcal{B}_{-q-k}(V)$ may be decomposed as $d_{\mathcal{B}}=\sum_{0 \leq j \leq q+k}(-1)^{j} d_{\mathcal{B}}^{j}$, where

$$
d_{\mathcal{B}}^{j}\left(b_{0}|\ldots| b_{k+q+1}\right):=\left(b_{0}|\ldots| b_{j-1}\left|b_{j} \cdot b_{j+1}\right| b_{j+2}|\ldots| b_{k+q+1}\right),
$$

for any $\left(b_{0}|\ldots| b_{k+q+1}\right) \in \mathcal{B}_{-q-k}(V)$. We may then write

$$
\begin{aligned}
& (-1)^{n-1} \cdot\left(\mathbb{1}^{\odot(r-1)} \odot d_{\mathcal{B}} \odot \mathbb{1}^{\odot(n-r)}\right) M^{r}[\sigma](\hat{a}) \\
& \quad=\sum S(-1)^{n+j-1} \cdot \operatorname{sgn}(\pi, \sigma) \cdot\left(\mathbb{1}^{\odot(r-1)} \odot d_{\mathcal{B}}^{j} \odot \mathbb{1}^{\odot(n-r)}\right) M_{\pi}^{r}[\sigma](\hat{a}),
\end{aligned}
$$

where $S:=\{(j, \pi) ; 0 \leq j \leq k+q, \pi \in P(q, k)\}$. 
Our proof of (19) involves distinguishing the various kinds of products which appear on the right side: products of two $a$ terms, products of an $a$ term and a $\Psi$ term in either order, and products of two $\Psi$ terms. We have the following disjoint decomposition of $S$ where the subscripts indicate the type of product associated to the pairs in a given set:

$$
S=S_{a \Psi}^{\prime} \cup S_{a \Psi}^{\prime \prime} \cup S_{\Psi a}^{\prime} \cup S_{\Psi a}^{\prime \prime} \cup S_{\Psi \Psi}^{1} \cup \cdots \cup S_{\Psi \Psi}^{k-1} \cup S_{a a},
$$

where

$$
\begin{aligned}
S_{a \Psi}^{\prime}:= & \{(j, \pi) \in S ; j=0, \pi(1)=1\}, \\
S_{\Psi a}^{\prime}:= & \{(j, \pi) \in S ; j=k+q, \pi(k)=q+1\}, \\
S_{a \Psi}^{\prime \prime}:= & \{(j, \pi) \in S ; \text { there exists } l, 1 \leq l \leq k, \\
& \text { s.t. } \pi(l)>\pi(l-1) \text { and } j=\pi(l)+l-2\} \backslash S_{a \Psi}^{\prime}, \\
S_{\Psi a}^{\prime \prime}:= & \{(j, \pi) \in S ; \text { there exists } l, 1 \leq l \leq k, \\
& \text { s.t. } \pi(l)<\pi(l+1) \text { and } j=\pi(l)+l-1\} \backslash S_{\Psi a}^{\prime}, \\
S_{\Psi \Psi}^{l}:= & \{(j, \pi) \in S ; \pi(l)=\pi(l+1), j=\pi(l)+l-1\}, 1 \leq l \leq k-1, \text { and } \\
S_{a a}:= & \{(j, \pi) \in S ; \text { there exists } l, 1 \leq l<k, \\
& \text { such that } \pi(l)+l-1<j<\pi(l+1)+l-1\} .
\end{aligned}
$$

Continuing this approach consider the second term on the right-hand side of (24). The differential $d_{\mathcal{B}}$ is applied to an element of $\mathcal{B}_{-q}(V)$, thus we may write

$$
M^{r}[\sigma]\left(d_{\mathcal{B}}(\hat{a})\right)=\sum_{T}(-1)^{j} \cdot \operatorname{sgn}(\pi, \sigma) \cdot M_{\pi}^{r}[\sigma]\left(d_{\mathcal{B}}^{j}(\hat{a})\right),
$$

where $T:=\{(j, \pi) ; 0 \leq j \leq q, \pi \in P(q-1, k)\}$. As for the term on the left-hand side of (24), we again have a decomposition $d_{S}=\sum_{0 \leq j \leq k}(-1)^{j} d_{S}^{j}$, where

$$
d_{S}^{j}\left[v_{0}, \ldots, v_{k}\right]:=\left[v_{0}, \ldots, v_{j-1}, v_{j+1}, \ldots, v_{k}\right], \text { for } 0 \leq j \leq k .
$$

We may write

$$
M^{r}\left[d_{S} \sigma\right](\hat{a})=\sum_{R}(-1)^{j} \cdot \operatorname{sgn}\left(\pi, d_{S}^{j}(\sigma)\right) \cdot M_{\pi}^{r}\left[d_{S}^{j} \sigma\right](\hat{a}),
$$

where $R:=\{(j, \pi) ; 0 \leq j \leq k, \pi \in P(q, k-1)\}$. We can decompose $R=$ $R^{0} \cup R^{1} \cup \cdots \cup R^{k}$ with $R^{l}:=\{(j, \pi) \in R ; j=l\}$.

The terms in (20) cancel in pairs arising from the isomorphisms (bijections) between the various sets of indices as described in the following two sublemmas.

Sublemma 5.8. There are isomorphisms $\alpha: S_{a \Psi}^{\prime \prime} \cong S_{\Psi a}^{\prime \prime}, \beta: T \cong S_{a a}, \gamma: R^{0} \cong$ $S_{a \Psi}^{\prime}, \delta: R^{k} \cong S_{\Psi a}^{\prime}$ and $\phi^{l}: R^{l} \cong S_{\Psi \Psi}^{l}, 1 \leq l \leq k-1$.

We give an explicit description of the isomorphisms. The isomorphism $\alpha: S_{a \Psi}^{\prime \prime} \cong$ $S_{\Psi a}^{\prime \prime}$ is given by $\alpha(j, \pi):=(j, \alpha(\pi))$, where

$$
\alpha(\pi)(s)= \begin{cases}\pi(s), & \text { for } i \neq l, \text { and } \\ \pi(s)-1, & \text { for } s=l .\end{cases}
$$

The isomorphism $\beta: T \cong S_{a a}, \beta(j, \pi):=(\beta(j), \beta(\pi))$ is given in the following way. For any $j, 0 \leq j \leq q$, there exists exactly one $l$ such that $\pi(l) \leq j \leq \pi(l+1)-1$. Define then $\beta(j):=j+l$ and

$$
\beta(\pi)(s)= \begin{cases}\pi(s), & \text { for } s \leq l, \text { and } \\ \pi(s)+1, & \text { for } s>l .\end{cases}
$$


The isomorphism $\gamma: R^{0} \cong S_{a \Psi}^{\prime}$ is defined as $\gamma(0, \pi):=(0, \gamma(\pi))$, where

$$
\gamma(\pi)(s)= \begin{cases}1, & \text { for } s=1, \text { and } \\ \pi(s-1), & \text { for } s \geq 2 .\end{cases}
$$

The isomorphism $\delta: R^{k} \cong S_{\Psi a}^{\prime}$ is defined as $\delta(k, \pi):=(k+q, \delta(\pi))$, where

$$
\delta(\pi)(s)= \begin{cases}\pi(s), & \text { for } s \leq k-1, \text { and } \\ q+1, & \text { for } s=k\end{cases}
$$

Finally, the isomorphism $\phi^{l}: R^{l} \cong S_{\Psi \Psi}^{l}$ is, for $1 \leq l \leq k-1$, defined by $\phi^{l}(l, \pi):=$ $\left(\pi(l)+l-1, \phi^{l}(\pi)\right)$, where

$$
\phi^{l}(\pi)(s)= \begin{cases}\pi(s), & \text { for } s \leq l, \text { and } \\ \pi(s-1), & \text { for } s>l .\end{cases}
$$

Lemma 5.7 will obviously follow from the next sublemma.

Sublemma 5.9. The following equations hold:

$$
\begin{aligned}
& \operatorname{sgn}(\pi, \sigma) \cdot\left(\mathbb{1}^{\odot(r-1)} \odot d_{\mathcal{B}}^{j} \odot \mathbb{1}^{\odot(n-r)}\right) M_{\pi}^{r}[\sigma](\hat{a}) \\
& \quad+\operatorname{sgn}(\alpha(\pi), \sigma) \cdot\left(\mathbb{1}^{\odot(r-1)} \odot d_{\mathcal{B}}^{j} \odot \mathbb{1}^{\odot(n-r)}\right) M_{\alpha(\pi)}^{r}[\sigma](\hat{a})=0,
\end{aligned}
$$

for $(j, \pi) \in S_{a \Psi}^{\prime \prime}$,

$$
\begin{aligned}
(-1)^{n+\beta(j)-1} \cdot \operatorname{sgn}(\beta(\pi), \sigma) & \cdot\left(\mathbb{1}^{\odot(r-1)} \odot d_{\mathcal{B}}^{\beta(j)} \odot \mathbb{1}^{\odot(n-r)}\right) M_{\beta(\pi)}^{r}[\sigma](\hat{a}) \\
+ & (-1)^{k+n+j} \cdot \operatorname{sgn}(\pi, \sigma) \cdot M_{\pi}^{r}[\sigma]\left(d_{\mathcal{B}}^{j}(\hat{a})\right)=0,
\end{aligned}
$$

for $(j, \pi) \in T$,

$$
\begin{aligned}
& \operatorname{sgn}\left(\pi, d_{S}^{0}(\sigma)\right) \cdot M_{\pi}^{r}\left[d_{S}^{0}(\sigma)\right](\hat{a}) \\
& \quad=(-1)^{n-1} \cdot \operatorname{sgn}(\gamma(\pi), \sigma) \cdot\left(\mathbb{1}^{\odot(r-1)} \odot d_{\mathcal{B}}^{0} \odot \mathbb{1}^{\odot(n-r)}\right) M_{\gamma(\pi)}^{r}[\sigma](\hat{a}),
\end{aligned}
$$

for $(0, \pi) \in R^{0}$,

$$
\begin{aligned}
(-1)^{k} \cdot \operatorname{sgn}\left(\pi, d_{S}^{k}(\sigma)\right) \cdot M_{\pi}^{r}\left[d_{S}^{k}(\sigma)\right](\hat{a})=(-1)^{n+k+q-1} & \\
\cdot & \operatorname{sgn}(\delta(\pi), \sigma) \cdot\left(\mathbb{1}^{\odot(r-1)} \odot d_{\mathcal{B}}^{k+q} \odot \mathbb{1}^{\odot(n-r)}\right) M_{\delta(\pi)}^{r}[\sigma](\hat{a}),
\end{aligned}
$$

for $(k, \pi) \in R^{k}$, and

$$
\begin{aligned}
& (-1)^{l} \cdot \operatorname{sgn}\left(\pi, d_{S}^{l}(\sigma)\right) \cdot M_{\pi}^{r}\left[d_{S}^{l}(\sigma)\right](\hat{a})=(-1)^{n+\pi(l)+l} \\
& \cdot \operatorname{sgn}\left(\phi^{l}(\pi), \sigma, \hat{a}\right) \cdot\left(\mathbb{1}^{\odot(r-1)} \odot d_{\mathcal{B}}^{\pi(l)+l-1} \odot \mathbb{1}^{\odot(n-r)} M_{\phi^{l}(\pi)}^{r}[\sigma](\hat{a}),\right.
\end{aligned}
$$

for $(l, \pi) \in R^{l}, 1 \leq l \leq k-1$.

The proof of the sublemma is straightforward but rather technical and will be postponed to the last section.

The next lemma is the key to proving that $m$ is a homomorphism of Lie algebras.

Lemma 5.10. Let $\sigma=\left[v_{0}, \ldots, v_{r}\right] \in C_{r}\left(K_{a}\right), \tau=\left[w_{0}, \ldots, w_{s}\right] \in C_{s}\left(K_{b}\right)$ and $\hat{a}=a_{0} \otimes \cdots \otimes a_{q+1} \in \mathcal{B}_{-q}(V)$. Then

$$
M^{l}\left[\sigma \diamond_{t} \tau\right](\hat{a})= \begin{cases}(-1)^{(a+1) s+(b+1) r} \cdot\left(\mathbb{1}^{\odot(t-1)} \odot M^{l-t+1}[\sigma] \odot \mathbb{1}^{\odot(b-t)}\right) M^{t}[\tau](\hat{a}), \\ 0, \quad \text { otherwise. } & l \in[t, t+a-1],\end{cases}
$$


We show first that this lemma would imply that

$$
m([\sigma, \tau])(\uparrow \hat{a})=[m(\sigma), m(\tau)](\uparrow \hat{a}),
$$

for any $\sigma, \tau$ and $\hat{a}$ as above, which would obviously finish the proof of Theorem 5.1. On the left-hand side of (31) we have

$$
\begin{gathered}
m([\sigma, \tau])(\uparrow \hat{a})=\sum_{1 \leq t \leq b}(-1)^{(a+1)(t+s+1)} \cdot m\left(\sigma \diamond_{t} \tau\right)(\uparrow \hat{a}) \\
-(-1)^{(a+r+1)(b+s+1)} \cdot \sum_{1 \leq t \leq a}(-1)^{(b+1)(t+r+1)} \cdot m\left(\tau \diamond_{t} \sigma\right)(\uparrow \hat{a}) \\
=\uparrow^{\odot(a+b-1)}\left\{\sum_{1 \leq t \leq b}(-1)^{(a+1)(t+s+1)} \cdot M\left[\sigma \diamond_{t} \tau\right](\hat{a})\right. \\
-(-1)^{(a+r+1)(b+s+1)} \\
\\
\left.\cdot \sum_{1 \leq t \leq a}(-1)^{(b+1)(t+r+1)} \cdot M\left[\tau \diamond_{t} \sigma\right](\hat{a})\right\} .
\end{gathered}
$$

On the other hand

$$
\begin{aligned}
& {[m(\sigma), m(\tau)](\uparrow \hat{a})} \\
& =[m(\sigma) \circ m(\tau)](\uparrow \hat{a})-(-1)^{(a+r+1)(b+s+1)} \cdot[m(\tau) \circ m(\sigma)](\uparrow \hat{a}) \\
& \left.=m(\sigma)(\uparrow \odot b M[\tau](\hat{a}))-(-1)^{(a+r+1)(b+s+1)} \cdot m(\tau)(\uparrow \odot a) M[\sigma](\hat{a})\right) \\
& =\uparrow \odot(a+b-1)\left\{\sum_{1 \leq t \leq b}(-1)^{(r+a+1)(t+1)+r(b+t)}\right. \\
& \quad-\left(\mathbb{1}^{\odot(t-1)} \odot M[\sigma] \odot \mathbb{1}^{\odot(b-t)}\right) M^{t}[\tau](\hat{a}) \\
& \sum_{1 \leq t \leq a}(-1)^{(a+r+t)(b+s+1)+s(a+t)} \\
& \left.\cdot\left(\mathbb{1}^{\odot t-1} \odot M[\sigma] \odot \mathbb{1}^{\odot a-t}\right) M^{t}[\sigma](\hat{a})\right\} .
\end{aligned}
$$

The signs which appear in the last two lines arise as follows.

$$
\begin{aligned}
& m(\sigma)(\uparrow \odot b M[\tau]) \\
&=\sum_{1 \leq t \leq b}\left(\mathbb{1}^{\odot(t-1)} \odot \uparrow^{\odot a} M[\sigma] \downarrow \odot \mathbb{1}^{\odot(b-t)}\right) \uparrow^{\odot b} M[\tau] \\
&=\quad / \text { switch } \uparrow^{\odot a} M[\sigma] \downarrow \text { and } \uparrow^{\odot(t-1)} / \\
&=\sum_{1 \leq t \leq b}(-1)^{(t+1)(r+a+1)} \cdot\left(\uparrow \odot(t-1) \odot \uparrow^{\odot a} M[\sigma] \odot \uparrow \odot(b-t)\right) M[\tau] \\
&=\quad / \text { switch } M[\sigma] \text { and } \uparrow \odot(b-t) / \\
&= \sum_{1 \leq t \leq b}(-1)^{(r+a+1)(t+1)+r(b+t)} \\
&= \quad \cdot\left(\uparrow \odot(t-1) \odot \uparrow^{\odot a} \odot \uparrow^{\odot(b-t)}\right)\left(\mathbb{1}^{\odot(t-1)} \odot M[\sigma] \odot \mathbb{1}^{\odot(b-t)}\right) M^{t}[\tau] \\
& \quad \sum_{1 \leq t \leq b}(-1)^{(r+a+1)(t+1)+r(b+t)} \\
& \quad \cdot\left(\mathbb{1}^{\odot(t-1)} \odot M[\sigma] \odot \mathbb{1}^{\odot(b-t)}\right) M^{t}[\tau](\hat{a}) .
\end{aligned}
$$

This explains the factor $(-1)^{r(b+t)}$ in the formula; the explanation of the second factor $(-1)^{s(a+t)}$ is the same. 
Summing (30) over $l$ we get

$$
\sum_{1 \leq t \leq b} M\left[\sigma \diamond_{t} \tau\right](\hat{a})=(-1)^{(a+1) s+(b+1) r} \cdot \sum_{1 \leq t \leq b}\left(\mathbb{1}^{\odot(t-1)} \odot M[\sigma] \odot \mathbb{1}^{\odot(b-t)}\right) M^{t}[\tau](\hat{a})
$$

while the same formula with the rôles of $\sigma$ and $\tau$ interchanged gives

$$
\sum_{1 \leq t \leq a} M\left[\tau \diamond_{t} \sigma\right](\hat{a})=(-1)^{(a+1) s+(b+1) r} \cdot \sum_{1 \leq t \leq a}\left(\mathbb{1}^{\odot(t-1)} \odot M[\tau] \odot \mathbb{1}^{\odot(a-t)}\right) M^{t}[\sigma](\hat{a})
$$

and (31) follows. In fact Lemma 5.10 implies a bit more, it says that the map $m$ preserves suitably defined comp structures.

Let us discuss the term on the left-hand side of (30). For $\phi \in \mathrm{Sh}_{r, s}$ denote (see the end of $\S 3$ for the notation)

$$
\sigma \diamond_{t, \phi} \tau:=\left[\left(v_{\phi^{\prime}(0)}, w_{\phi^{\prime \prime}(0)}\right)_{t}, \ldots,\left(v_{\phi^{\prime}(r+s)}, w_{\phi^{\prime \prime}(r+s)}\right)_{t}\right] \in C_{r+s}\left(K_{a+b-1}\right) .
$$

Then

$$
M^{l}\left[\sigma \diamond_{t} \tau\right](\hat{a})=\sum_{U} \operatorname{sgn}\left(\pi, \sigma \diamond_{t, \phi} \tau\right) \cdot \operatorname{sgn}(\phi) \cdot M_{\pi}^{l}\left[\sigma \diamond_{t, \phi} \tau\right](\hat{a}),
$$

where $U:=\left\{(\phi, \pi) ; \phi \in \mathrm{Sh}_{r, s}, \pi \in P(q, r+s)\right\}$. On the other hand,

$$
\begin{aligned}
& \left(\mathbb{1}^{\odot t-1} \odot M^{l-t+1}[\sigma] \odot \mathbb{1}^{\odot b-t}\right) M^{t}[\tau](\hat{a}) \\
& \quad=\sum_{V} \operatorname{sgn}\left(\pi^{\prime}, \sigma,\right) \cdot \operatorname{sgn}\left(\pi^{\prime \prime}, \tau\right) \cdot\left(\mathbb{1}^{\odot t-1} \odot M_{\pi^{\prime}}^{l-t+1}[\sigma] \odot \mathbb{1}^{\odot b-t}\right) M_{\pi^{\prime \prime}}^{t}[\tau](\hat{a}),
\end{aligned}
$$

with $V:=\left\{\left(\pi^{\prime}, \pi^{\prime \prime}\right) ; \pi^{\prime} \in P(q+s, r), \pi^{\prime \prime} \in P(q, s)\right\}$. Let us construct an isomorphism $\chi: V \cong U$ as follows. Let $\pi^{\prime \prime} \in P(q, s)$ and let, just for the moment, $a_{0}, \ldots, a_{q+1}$ be a sequence of independent symbols. Consider the sequence

$$
\left(b_{0}, \ldots, b_{s+q+1}\right):=\left(a_{0}, \ldots, a_{\pi^{\prime \prime}(1)-1}, \Psi_{1}, a_{\pi^{\prime \prime}(1)}, \ldots, a_{\pi^{\prime \prime}(s)-1}, \Psi_{s}, a_{\pi^{\prime \prime}(s)}, \ldots, a_{q+1}\right),
$$

where $\Psi_{1}, \ldots, \Psi_{s}$ are other independent symbols. Let $\pi^{\prime} \in P(q+s, r)$ and define

$$
\begin{aligned}
& \left(c_{0}, \ldots, c_{r+s+q+1}\right) \\
& \quad:=\left(b_{0}, \ldots, b_{\pi^{\prime}(1)-1}, \bar{\Psi}_{1}, b_{\pi^{\prime}(1)}, \ldots, b_{\pi^{\prime}(r)-1}, \bar{\Psi}_{r}, b_{\pi^{\prime}(s)}, \ldots, b_{s+q+1}\right),
\end{aligned}
$$

for a sequence $\bar{\Psi}_{1}, \ldots, \bar{\Psi}_{s}$ of independent symbols. We may then write

$$
\begin{aligned}
& \left(c_{0}, \ldots, c_{r+s+q+1}\right) \\
& \quad=\left(a_{0}, \ldots, a_{\pi(1)-1}, X_{1}, a_{\pi(1)}, \ldots, a_{\pi(r+s)-1}, X_{r+s}, a_{\pi(r+s)}, \ldots, a_{q+1}\right)
\end{aligned}
$$

for some $\pi \in P(q, r+s)$ and $X_{h} \in\left\{\Psi_{1}, \ldots, \Psi_{s}, \bar{\Psi}_{1}, \ldots, \bar{\Psi}_{r}\right\}, 1 \leq h \leq r+s$. Define then

$$
\left\{i_{1}<\cdots<i_{r}\right\}:=\left\{h ; X_{h}=\bar{\Psi}_{h}\right\} \text { and }\left\{j_{1}<\cdots<j_{s}\right\}:=\left\{h ; X_{h}=\Psi_{h}\right\} .
$$

These data determine a shuffle $\phi \in \mathrm{Sh}_{r, s}$. Put $\chi\left(\pi^{\prime}, \pi^{\prime \prime}\right):=(\phi, \pi)$. The description of $\pi$ in terms of $\pi^{\prime}$ and $\pi^{\prime \prime}$ is as follows. Let

$$
\bar{i}_{k}=\max \left\{i \mid \pi^{\prime \prime}(i)+i \leq \pi^{\prime}(k)\right\},
$$

then the sequence $\pi(i), 1 \leq i \leq r+s$, is given by

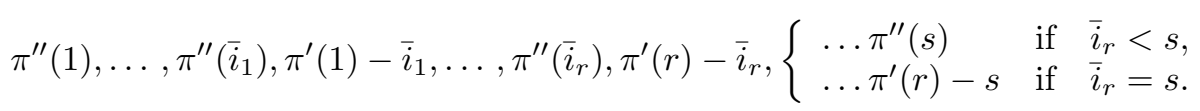


The corresponding shuffle $\phi$ is given by those indices for which the related associativity operator has the form $1^{\otimes c} \otimes \Psi_{v, v^{\prime}} \otimes 1^{\otimes c^{\prime}}$. These are the indices $i$ where $\pi(i)=\pi^{\prime}(a)-\bar{i}_{a}, i_{a}=\bar{i}_{a}+a$. Then

$$
\operatorname{sgn}(\phi)=(-1)^{\sum i_{a}-a}=(-1)^{\sum \bar{i}_{a}} .
$$

Lemma 5.10 would then follow from the following sublemma.

Sublemma 5.11. $M_{\pi}^{l}\left[\sigma \diamond_{t, \phi} \tau\right](\hat{a})=0$ for $l \notin[t, t+a-1], \phi \in \mathrm{Sh}_{r, s}$ and $\pi \in$ $P(q, r+s)$, while

$$
\begin{aligned}
\operatorname{sgn}\left(\pi, \sigma \diamond_{t, \phi} \tau\right) \cdot \operatorname{sgn}(\phi) \cdot M_{\pi}^{l}\left[\sigma \diamond_{t, \phi} \tau\right](\hat{a}) & \\
= & (-1)^{(a+1) s+(b+1) r} \cdot \operatorname{sgn}\left(\pi^{\prime}, \sigma\right) \cdot \operatorname{sgn}\left(\pi^{\prime \prime}, \tau\right) \\
& \cdot\left(\mathbb{1}^{\odot(t-1)} \odot M_{\pi^{\prime}}^{l-t+1}[\sigma] \odot \mathbb{1}^{\odot(b-t)}\right) M_{\pi^{\prime \prime}}^{t}[\tau](\hat{a})
\end{aligned}
$$

for $(\phi, \pi)=\chi\left(\pi^{\prime}, \pi^{\prime \prime}\right),\left(\pi^{\prime}, \pi^{\prime \prime}\right) \in V$ and $l \in[t, t+a-1]$.

The proof, technical although straightforward, will be given in the last section.

\section{Cohomology and the Deformations}

As we have already observed, both $\odot(V, \mathcal{B}(V))^{*}(=\bigodot(\uparrow V \oplus \uparrow \mathcal{B}(V)))$ and $\bigodot^{*}(V)\left(=\bigodot(\uparrow V)^{*}\right)$ are graded $(V, \mu)$-modules (via the $\bullet$-action). The $\odot$-multiplication yields on both $\odot(V, \mathcal{B}(V))^{*}$ and $\bigodot^{*}(V)$ the structures of graded $\bigodot^{*}(V)$-bimodules and both structures are related by $(4)$. Let $\bigodot^{\prime}(V, \mathcal{B}(V))=$ $\bigodot^{*, 1}(V, \mathcal{B}(V))$ be the submodule with precisely one factor of $\mathcal{B}(V)$. Denote by $\operatorname{Hom}^{n}\left(\bigodot^{\prime}(V, \mathcal{B}(V))^{*}, \bigodot^{*}(V)\right)$ the set of all degree $n$ homogeneous maps $f: \bigodot^{\prime}(V, \mathcal{B}(V))^{*} \rightarrow \bigodot^{*}(V)$ which are both $\bigodot^{*}(V)$ and $(V, \mu)$-linear. For such a map put $d(f):=f \circ D+(-1)^{n} d_{C} \circ f$, where $D$ is as in Corollary 5.2 and $d_{C}$ is the degree one derivation on $\bigodot^{*}(V)$ defined by $\left.d_{C}\right|_{\uparrow V}:=(\uparrow \odot \uparrow) \Delta \downarrow$.

Definition 6.1. The cohomology of a Drinfel'd algebra $A=(V, \cdot, \Delta, \Phi)$ is the graded vector space

$$
H^{*}(A):=H^{*}\left(C^{*}(A), d\right)
$$

where $C^{*}(A):=\operatorname{Hom}^{*}\left(\bigodot^{\prime}(V, \mathcal{B}(V))^{*}, \bigodot^{*}(V)\right)$ and the differential $d$ is as above.

Let us try to understand better the structure of the cohomology above. Using the fact that $\bigodot^{\prime}(V, \mathcal{B}(V))^{*}$ is, in a sense, the free $\bigodot^{*}(V)$-bimodule on the $(V, \mu)$ bimodule $\uparrow \mathcal{B}_{*}(V)$, we may write

$$
\operatorname{Hom}^{n}\left(\bigodot^{\prime}(V, \mathcal{B}(V))^{*}, \bigodot^{*}(V)\right) \cong \operatorname{Hom}_{V}^{n+1}\left(\mathcal{B}_{*}(V), \bigodot^{*}(V)\right),
$$

where $\operatorname{Hom}_{V}^{n+1}\left(\mathcal{B}_{*}(V), \bigodot^{*}(V)\right)$ denotes the set of $(V, \mu)$-linear maps $f: \mathcal{B}_{*}(V) \rightarrow$ $\odot^{*}(V)$ of degree $(n+1)$. Using the fact that $\mathcal{B}_{*}(V)$ is the free $(V, \mu)$-module on $\bigotimes^{-*}(\bar{V})$ (recall that $\bar{V}=\operatorname{Ker}(\epsilon)$ ), we get the identification

$$
\operatorname{Hom}_{V}^{n+1}\left(\mathcal{B}_{*}(V), \bigodot^{*}(V)\right) \cong \operatorname{Hom}_{\mathbf{k}}^{n+1}\left(\bigotimes^{-*}(\bar{V}), \bigodot^{*}(V)\right)
$$

where $\operatorname{Hom}_{\mathbf{k}}^{n+1}\left(\bigotimes^{-*}(\bar{V}), \bigodot^{*}(V)\right)$ denotes the set of degree $(n+1) \mathbf{k}$-linear homogeneous maps $g: \bigotimes^{-*}(\bar{V}) \rightarrow \bigodot^{*}(V)$. Finally, invoking the canonical isomorphism $J: \bigodot^{*}(V) \cong \bigotimes^{*}(V)$ of graded vector spaces constructed in [13, Proposition 3.3] we get

$$
\operatorname{Hom}_{\mathbf{k}}^{n+1}\left(\bigotimes^{-*}(\bar{V}), \bigodot^{*}(V)\right) \cong \operatorname{Hom}_{\mathbf{k}}^{n+1}\left(\bigotimes^{-*}(\bar{V}), \bigotimes^{*}(V)\right)
$$


Summing up the above remarks, we get the canonical identification

$$
C^{n}(A) \cong \operatorname{Hom}_{\mathbf{k}}^{n+1}\left(\bigotimes^{-*}(\bar{V}), \bigotimes *(V)\right), n \geq 0
$$

This is the underlying vector space of the Gerstenhaber-Schack complex [8] which controls the deformation of coassociative bialgebras. In fact, our complex can be considered as a kind of deformation of the Gerstenhaber-Schack complex as follows.

Let us simplify the notation by setting $E^{i, j}(A):=\operatorname{Hom}_{\mathbf{k}}\left(\bigotimes^{j}(\bar{V}), \bigotimes^{i}(V)\right)$. Then it is immediate to see that $C^{n}(A)=\bigoplus_{i+j=n+1} E^{i, j}(A)$ and that the differential $d$ decomposes as $d=d_{\text {Hoch }}+d_{\mathrm{q}-\mathrm{Car}}+\sum_{k=1}^{\infty} d_{k}$, where

$$
\begin{gathered}
f \mapsto f \circ D_{-1} \quad \text { induces } \quad d_{\mathrm{Hoch}}: E^{*, *}(A) \rightarrow E^{*, *+1}(A), \\
f \mapsto f \circ D_{0}+(-1)^{|f|} \cdot d_{C} \circ f \quad \text { induces } \quad d_{\mathrm{q}-\mathrm{Car}}: E^{*, *}(A) \rightarrow E^{*+1, *}(A) \\
\text { and } \quad f \mapsto f \circ D_{k} \quad \text { induces } \quad d_{k}: E^{*, *}(A) \rightarrow E^{*+k+1, *-k}(A), k \geq 1 .
\end{gathered}
$$

Here $d_{\text {Hoch }}$ is the differential of the Hochschild complex of the associative algebra $(V, \cdot)$ with coefficients in the bimodule $\bigotimes^{*}(V), d_{\mathrm{q}-\mathrm{Car}}$ is the analog of the Cartier differential (see [2]) for the (noncoassociative) coalgebra $(V, \Delta)$, and the maps $d_{k}$ correspond to the derivations $D_{k}$ of Corollary 5.2, for $k \geq 1$. Bigraded differential complexes where the differential decomposes in as in (33) are sometimes called "multicompexes" (although the first author prefers to call them monsters).

In general, when $\Phi \neq 1$, we do not have $d_{\mathrm{q}-\mathrm{Car}}^{2}=0$. However when $\Phi=1$, $d_{\mathrm{q}-\mathrm{Car}}^{2}=0$ and $d_{\text {Hoch }}+d_{\mathrm{q}-\mathrm{Car}}$ is the differential defined in [8]. Moreover, in this case the operators $D_{k}$ vanish, which easily follows from the fact that we work with normalized bar resolution. So the cohomology of our complex is the same as that of the Gerstenhaber-Schack complex for the bialgebra cohomology. Notice also that the complex $\left(E^{*, 0}(A), d_{\mathrm{q}-\mathrm{Car}}\right)$ is exactly the $M$-construction $\left(M^{*}, d_{q \Omega}\right)$ of $[13$, par. 3].

Now we arrive at our goal of defining a cohomology theory controlling the deformations of Drinfel'd algebras. The meaning of a "cohomology theory controlling the deformations" has been explained in the introduction. For more details we refer the reader to the classical exposition [5] or more recent treatments $[4,10,15]$.

Theorem 6.2. Deformations of a Drinfel'd algebra $A=(V, \cdot, \Delta, \Phi)$ are controlled by the cohomology

$$
\hat{H}^{*}(A):=H^{*}(\hat{C}(A), \hat{d}),
$$

where $\hat{C}^{n}(A):=\bigoplus\left\{E^{i, j}(A) ; i+j=n+1, i \geq 1\right\}$ and $\hat{d}:=\left.d\right|_{\hat{C}(A)}$.

Proof. Observe first that

$$
\begin{aligned}
& \hat{C}^{1}(A)=\operatorname{Hom}_{\mathbf{k}}(\bar{V}, V) \oplus V^{2}, \\
& \hat{C}^{2}(A)=\operatorname{Hom}_{\mathbf{k}}\left(\bigotimes^{2} \bar{V}, V\right) \oplus \operatorname{Hom}_{\mathbf{k}}\left(\bar{V}, \bigotimes^{2} V\right) \oplus V^{3}, \text { and } \\
& \hat{C}^{3}(A)=\operatorname{Hom}_{\mathbf{k}}\left(\bar{V}, \bigotimes^{3} V\right) \oplus \operatorname{Hom}_{\mathbf{k}}\left(\bigotimes^{2} \bar{V}, \bigotimes^{2} V\right) \oplus \operatorname{Hom}_{\mathbf{k}}\left(\bar{V}, \bigotimes^{3} V\right) \oplus V^{4}
\end{aligned}
$$


Let $h=(\chi, D, \Psi) \in \hat{C}^{2}(A)$ be an infinitesimal deformation. By definition, $\hat{d}(h)=0$ is equivalent to

$$
\begin{aligned}
d_{\text {Hoch }}(\chi) & =0 \text { in } \operatorname{Hom}_{\mathbf{k}}\left(\bigotimes^{3} \bar{V}, V\right), \\
d_{\mathrm{q}-\mathrm{Car}}(\chi)+d_{\mathrm{Hoch}}(D) & =0 \text { in } \operatorname{Hom}_{\mathbf{k}}\left(\bigotimes^{2} \bar{V}, \bigotimes^{2} V\right), \\
d_{1}(\chi)+d_{\mathrm{q}-\mathrm{Car}}(D)+d_{\mathrm{Hoch}}(\Psi) & =0 \text { in } \operatorname{Hom}_{\mathbf{k}}\left(\bar{V}, \bigotimes^{3} V\right), \text { and } \\
d_{2}(\chi)+d_{1}(D)+d_{\mathrm{q}-\mathrm{Car}}(\Psi) & =0 \text { in } \operatorname{Hom}_{\mathbf{k}}\left(\mathbf{k}, \bigotimes^{4} V\right) .
\end{aligned}
$$

We shall see that these are exactly the infinitesimal forms of the structure equations, (33) is the infinitesimal form of the associativity of $\mu,(34)$ is the infinitesimal form of the compatibility between $\mu$ and $\Delta$, (35) is the infinitesimal form of the quasi-coassociativity (1) and (36) is the infinitesimal form of the pentagon identity (2). From this it will follow that $\hat{d}(h)=0$ if and only if $(\mu+t \chi, \Delta+t D, \Phi+t \Psi)$ is an infinitesimal deformation of the algebra $A=(V, \mu, \Delta, \Phi), t$ being an independent variable.

Suppose $g=(\phi, F) \in \hat{C}^{1}(A)$. By definition, $\hat{d}(g)=h$ means that

$$
\begin{aligned}
d_{\text {Hoch }}(\phi) & =\chi, \\
d_{\mathrm{q}-\operatorname{Car}}(\phi)+d_{\text {Hoch }}(F) & =D, \\
d_{1}(\phi)+d_{\mathrm{q}-\operatorname{Car}}(F) & =\Psi .
\end{aligned}
$$

We shall show that the above equations describe the infinitesimal action of the infinitesimal automorphism $\phi$ and the infinitesimal twisting $F$ on the infinitesimal deformation $(\chi, D, \Psi)$, hence $\hat{H}^{2}(A)$ describes the infinitesimal deformations modulo infinitesimal automorphisms and twistings. A first order deformation is equivalent to the trivial first order deformation if and only if the infinitesimal cocycle cobounds and two extensions (which both cobound the same obstruction cocycle) are equivalent if and only if their difference cobounds.

We now proceed to demonstrate the statements made above by giving the explicit formulae for the terms of (33) to (39), separating the terms according to the type of operator, see Figure 8 for the positions of relevant elements in the bicomplex.

We use the same convention as in the introduction, i.e. we represent our bigraded complex as a non-negative integer lattice in the plane with the position $i$ th column $j$ th row (counting upwards) occupied by $E^{i, j}(A)$. First we describe the Hochschild differentials represented in Figure 9.

The differential $d_{\text {Hoch }}: \operatorname{Hom}_{\mathbf{k}}\left(\otimes^{2} \bar{V}, V\right) \rightarrow \operatorname{Hom}_{\mathbf{k}}\left(\bigotimes^{3} \bar{V}, V\right)$ is given by

$$
d_{\text {Hoch }}(\chi)\left(v_{1}, v_{2}, v_{3}\right)=v_{1} \cdot \chi\left(v_{2}, v_{3}\right)-\chi\left(v_{1} \cdot v_{2}, v_{3}\right)+\chi\left(v_{1}, v_{2} \cdot v_{3}\right)-\chi\left(v_{1}, v_{2}\right) \cdot v_{3} \text {, }
$$

for $\chi \in \operatorname{Hom}_{\mathbf{k}}\left(\otimes^{2} \bar{V}, V\right), v_{1}, v_{2}, v_{3} \in \bar{V}$. This is the variational derivative of the associativity expression $\left(v_{1} v_{2}\right) v_{3}-v_{1}\left(v_{2} v_{3}\right)$ for the deformed multiplication relative to an infinitesimal change in the multiplication. It appears in (33) and its vanishing is the usual cocycle condition for infinitesimal deformation of an associative multiplication.

The differential $d_{\text {Hoch }}: \operatorname{Hom}_{\mathbf{k}}\left(\bar{V}, \bigotimes^{2} V\right) \rightarrow \operatorname{Hom}_{\mathbf{k}}\left(\bigotimes^{2} \bar{V}, \bigotimes^{2} V\right)$ is given by

$$
\begin{aligned}
d_{\text {Hoch }}(D)= & \sum D^{\prime}\left(v_{1}\right) \cdot \Delta^{\prime}\left(v_{2}\right) \otimes D^{\prime \prime}\left(v_{1}\right) \cdot \Delta^{\prime \prime}\left(v_{2}\right) \\
& -D\left(v_{1} \cdot v_{2}\right)+\sum \Delta^{\prime}\left(v_{1}\right) \cdot D^{\prime}\left(v_{2}\right) \otimes \Delta^{\prime \prime}\left(v_{1}\right) \cdot D^{\prime \prime}\left(v_{2}\right),
\end{aligned}
$$


Figure 8. Positions in $E^{* *}$ of infinitesimal automorphism $\phi$, twisting $F$, infinitesimal deformations $\chi, D, \Psi$ of multiplication, comultiplication, and $\Phi$, respectively, and obstruction classes $\Omega_{a}$, $\Omega_{c}, \Omega_{q}$ and $\Omega_{p}$ of the structure equations, associativity, compatibility, quasi-associativity and pentagon identities, respectively.

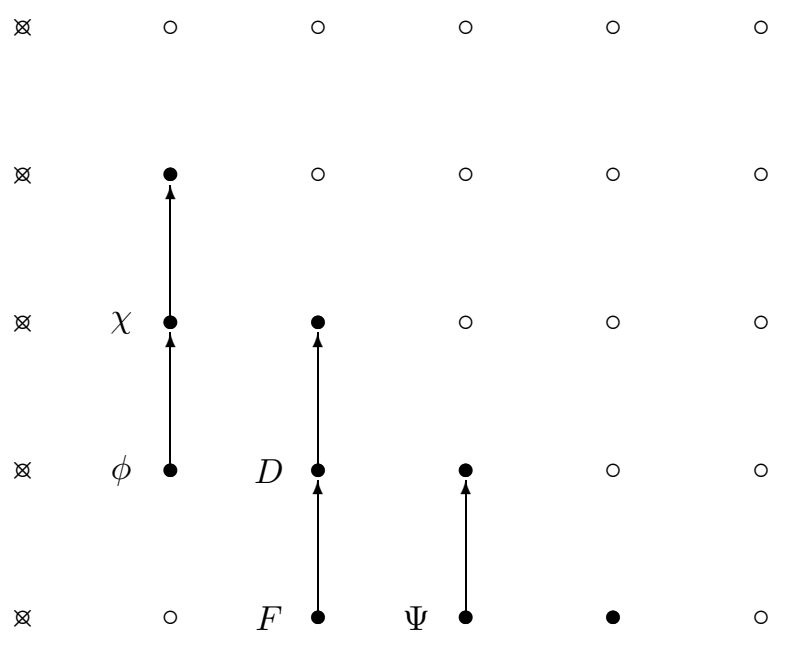

Figure 9. The Hochschild differentials of representative elements.

for $D=\sum D^{\prime} \otimes D^{\prime \prime} \in \operatorname{Hom}_{\mathbf{k}}\left(\bar{V}, \otimes^{2} V\right), \Delta=\sum \Delta^{\prime} \otimes \Delta^{\prime \prime}$ (the Sweedler notation) and $v_{1}, v_{2} \in \bar{V}$. This is the variational derivative of the condition for compatibility of multiplication and comultiplication with respect to an infinitesimal change in the comultiplication, $D$. It is one of the summands in (34). We complete the discussion of this and the remaining equations once we have described all the summands in a particular equation. 
The differential $d_{\text {Hoch }}: \operatorname{Hom}_{\mathbf{k}}\left(\mathbf{k}, \bigotimes^{3} V\right) \rightarrow \operatorname{Hom}_{\mathbf{k}}\left(\bar{V}, \bigotimes^{3} V\right)$ is given by

$$
d_{\text {Hoch }}(\Psi)(v)=(\mathbb{1} \otimes \Delta) \Delta(v) \cdot \Psi-\Psi \cdot(\Delta \otimes \mathbb{1}) \Delta(v),
$$

for $\Psi \in \operatorname{Hom}_{\mathbf{k}}\left(\mathbf{k}, \otimes^{3} V\right)$ and $v \in \bar{V}$. This is the variational derivative of the quasicoassociativity condition relative to an infinitesimal variation, $\Psi$, in the (co)associativity operator $\Phi$ of the Drinfel'd algebra.

The differential $d_{\text {Hoch }}: \operatorname{Hom}_{\mathbf{k}}(\bar{V}, V) \rightarrow \operatorname{Hom}_{\mathbf{k}}\left(\otimes^{2} \bar{V}, V\right)$ is given by

$$
d_{\text {Hoch }}(\phi)\left(v_{1}, v_{2}\right)=v_{1} \cdot \phi\left(v_{2}\right)-\phi\left(v_{1} \cdot v_{2}\right)+\phi\left(v_{1}\right) \cdot v_{2},
$$

for $\phi \in \operatorname{Hom}_{\mathbf{k}}(\bar{V}, V), v_{1}, v_{2} \in \bar{V}$. This is the variational derivative of multiplication relative to an infinitesimal automorphism. Just as in the classical theory of deformations of associative algebras, if the infinitesimal deformation of multiplication is the Hochschild coboundary of $\phi$, then the automorphism $i d+t \phi$ transforms it to zero.

The differential $d_{\text {Hoch }}: \operatorname{Hom}_{\mathbf{k}}\left(\mathbf{k}, \bigotimes^{2} V\right) \rightarrow \operatorname{Hom}_{\mathbf{k}}\left(\bar{V}, \bigotimes^{2} V\right)$ is given by

$$
d_{\text {Hoch }}(F)(a)=F \cdot \Delta(a)-\Delta(a) \cdot F
$$

for $F \in \operatorname{Hom}_{\mathbf{k}}\left(\mathbf{k}, \bigotimes^{2} V\right)=\bigotimes^{2} V$ and $a \in \bar{V}$. This expresses the variational derivative of comultiplication with respect to an infinitesimal twisting, $F$, which appears in (38) and will be discussed below.

Next consider the operator $d_{\mathrm{q}-\mathrm{Car}}$ on the same elements, see Figure 10. The operator $d_{\mathrm{q}-\mathrm{Car}}: \operatorname{Hom}_{\mathbf{k}}\left(\otimes^{2} \bar{V}, V\right) \rightarrow \operatorname{Hom}_{\mathbf{k}}\left(\otimes^{2} \bar{V}, \bigotimes^{2} V\right)$ is given by

$$
\begin{aligned}
d_{\mathrm{q}-\mathrm{Car}}(\chi)\left(v_{1}, v_{2}\right)= & \sum \Delta^{\prime}\left(v_{1}\right) \cdot \Delta^{\prime}\left(v_{2}\right) \otimes \chi\left(\Delta^{\prime \prime}\left(v_{1}\right), \Delta^{\prime \prime}\left(v_{2}\right)\right)-\Delta \chi\left(v_{1}, v_{2}\right) \\
& +\sum \chi\left(\Delta^{\prime}\left(v_{1}\right), \Delta^{\prime}\left(v_{2}\right)\right) \otimes \Delta^{\prime \prime}\left(v_{1}\right) \cdot \Delta^{\prime \prime}\left(v_{2}\right),
\end{aligned}
$$

for $\chi \in \operatorname{Hom}_{\mathbf{k}}\left(\otimes^{2} \bar{V}, V\right), \Delta=\sum \Delta^{\prime} \otimes \Delta^{\prime \prime}$ and $v_{1}, v_{2} \in \bar{V}$. This gives the variational derivative of the compatibility condition for multiplication and comultiplication relative to an infinitesimal variation, $\chi$, in multiplication. Equation (34) says that the total variational derivative of the compatibility condition relative to a combined infinitesimal variation of multiplication and comultiplication is zero. This is clearly a necessary and sufficient condition for the infinitesimal preservation of compatibility.

The operator $d_{\mathrm{q}-\mathrm{Car}}: \operatorname{Hom}_{\mathbf{k}}\left(\bar{V}, \bigotimes^{2} V\right) \rightarrow \operatorname{Hom}_{\mathbf{k}}\left(\bar{V}, \bigotimes^{3} V\right)$ is given by

$d_{\mathrm{q}-\operatorname{Car}}(D)(v)=(\mathbb{1} \otimes D) \Delta(v) \cdot \Phi-\Phi \cdot(\Delta \otimes \mathbb{1}) D(v)+(\mathbb{1} \otimes \Delta) D(v) \cdot \Phi-\Phi \cdot(D \otimes \mathbb{1}) \Delta(v)$,

for $D \in \operatorname{Hom}_{\mathbf{k}}\left(\bar{V}, \bigotimes^{2} V\right), v \in \bar{V}$, which is the variational derivative of the quasicoassociativity relative to an infinitesimal change in comultiplication. It appears in (35) and we need to consider yet one more term before concluding the discussion of this equation.

The operator $d_{\mathrm{q}-\mathrm{Car}}: \operatorname{Hom}_{\mathbf{k}}\left(\mathbf{k}, \bigotimes^{3} V\right) \rightarrow \operatorname{Hom}_{\mathbf{k}}\left(\mathbf{k}, \bigotimes^{4} V\right)$ is given by

$$
\begin{aligned}
d_{\mathrm{q}-\mathrm{Car}}(\Psi)= & (1 \otimes \Psi) \cdot(\mathbb{1} \otimes \Delta \otimes \mathbb{1})(\Phi) \cdot(\Phi \otimes 1)-\left(\mathbb{1}^{2} \otimes \Delta\right)(\Phi) \cdot\left(\Delta \otimes \mathbb{1}^{2}\right)(\Psi) \\
& +(1 \otimes \Phi) \cdot(\mathbb{1} \otimes \Delta \otimes \mathbb{1})(\Psi) \cdot(\Phi \otimes 1)-\left(\mathbb{1}^{2} \otimes \Delta\right)(\Psi) \cdot\left(\Delta \otimes \mathbb{1}^{2}\right)(\Phi) \\
& +(1 \otimes \Phi) \cdot(\mathbb{1} \otimes \Delta \otimes \mathbb{1})(\Phi) \cdot(\Psi \otimes 1)
\end{aligned}
$$

for $\Psi \in \operatorname{Hom}_{\mathbf{k}}\left(\mathbf{k}, \bigotimes^{3} V\right)=\bigotimes^{3} V$. It represents the variational derivative of the pentagon identity relative to an infinitesimal variation, $\Psi$, in $\Phi$. 


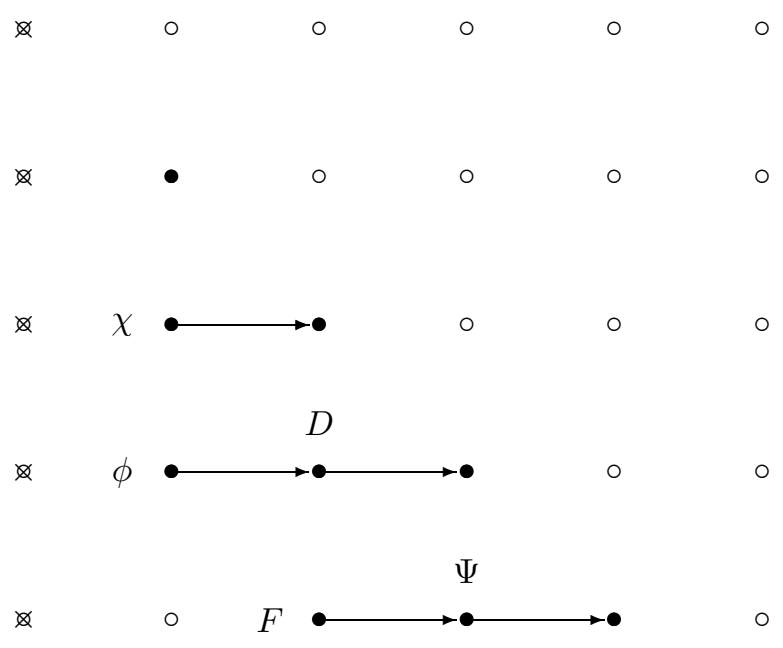

Figure 10. The modified Cartier operator on representative elements.

○ $\quad 0$

$\circ$

$\circ \quad \circ$

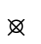

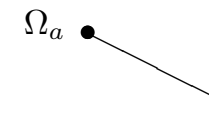

$\not$

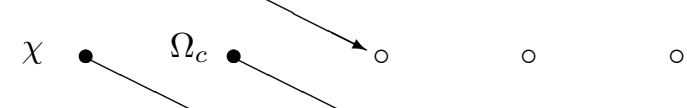

$\varnothing$

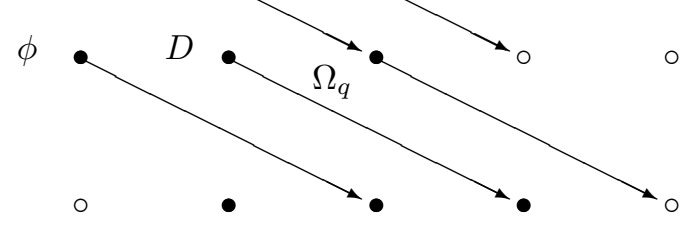

Figure 11. All the occurrences of the operator $d_{1}$ in deformation theory.

The operator $d_{\mathrm{q}-\mathrm{Car}}: \operatorname{Hom}_{\mathbf{k}}(\bar{V}, V) \rightarrow \operatorname{Hom}_{\mathbf{k}}\left(\bar{V}, \bigotimes^{2} V\right)$ for $\phi \in \operatorname{Hom}_{\mathbf{k}}(\bar{V}, V)$ and $v \in \bar{V}$ is given by

$$
d_{\mathrm{q}-\mathrm{Car}}(\phi)(v)=(\mathbb{1} \otimes \phi) \Delta(v)-\Delta \phi(v)+(\phi \otimes \mathbb{1}) \Delta,
$$

which expresses the variational derivative of the comultiplication relative to an infinitesimal automorphism, $\phi$. The sum of this term and $d_{\text {Hoch }}(F)$ is the total variational derivative of the comultiplication relative to a combined infinitesimal automorphism and twisting. If this gives the infinitesimal comultiplication, then the combined effect of the automorphism $\mathbb{1}+t \phi$ and twisting by $1+t F$ is to transform the infinitesimal comultiplication to zero.

The operator $d_{\text {q-Car }}: \operatorname{Hom}_{\mathbf{k}}\left(\mathbf{k}, \bigotimes^{2} V\right) \rightarrow \operatorname{Hom}_{\mathbf{k}}\left(\mathbf{k}, \bigotimes^{3} V\right)$ is given by

$$
d_{\mathrm{q}-\mathrm{Car}}(F)=(1 \otimes F) \cdot \Phi-\Phi \cdot(\Delta \otimes \mathbb{1})(F)+(\mathbb{1} \otimes \Delta)(F) \cdot \Phi-\Phi \cdot(F \otimes 1),
$$


for $F \in \operatorname{Hom}_{\mathbf{k}}\left(\mathbf{k}, \bigotimes^{2} V\right)=\bigotimes^{2} V$. This is the variational derivative of the (co)associativity operator $\Phi$ relative to an infinitesimal twisting, $F$.

We shall give the explicit formula for three of the six occurrences of $d_{1}$ in deformation theory as illustrated in Figure 11.

The variational derivative of the quasi-coassociativity relative to an infinitesimal change in multiplication, $\chi$, is given by the map $d_{1}: \operatorname{Hom}_{\mathbf{k}}\left(\otimes^{2} \bar{V}, V\right) \rightarrow$ $\operatorname{Hom}_{\mathbf{k}}\left(\bar{V}, \bigotimes^{3} V\right)$,

$$
\begin{array}{r}
d_{1}(\chi)=\sum_{\left(\bigcirc_{1}, \bigcirc_{2}, \bigcirc_{3}\right) \in A}\left\{\sum\left(\bigcirc_{1} \otimes \bigcirc_{2} \otimes \bigcirc_{3}\right)((\mathbb{1} \otimes \Delta) \Delta(v) \bar{\otimes} \Phi\right. \\
-\Phi \bar{\otimes}(\Delta \otimes \mathbb{1}) \Delta(v))\} \\
=\sum_{\left(\bigcirc_{1}, \bigcirc_{2}, \bigcirc_{3}\right) \in A}\left\{\sum \bigcirc_{1}\left(\Delta^{\prime}(v), \Phi_{1}\right) \otimes \bigcirc_{2}\left(\Delta^{\prime}\left(\Delta^{\prime \prime}(v)\right), \Phi_{2}\right)\right. \\
\otimes \bigcirc_{3}\left(\Delta^{\prime \prime}\left(\Delta^{\prime \prime}(v)\right), \Phi_{3}\right) \\
-\sum \bigcirc_{1}\left(\Phi_{1}, \Delta^{\prime}\left(\Delta^{\prime}(v)\right)\right) \otimes \bigcirc_{2}\left(\Phi_{2}, \Delta^{\prime \prime}\left(\Delta^{\prime}(v)\right)\right) \\
\left.\otimes \bigcirc_{3}\left(\Phi_{3}, \Delta^{\prime \prime}(v)\right)\right\},
\end{array}
$$

for $\chi \in \operatorname{Hom}_{\mathbf{k}}\left(\otimes^{2} \bar{V}, V\right), v \in \bar{V}, \Phi=\sum \Phi_{1} \otimes \Phi_{2} \otimes \Phi_{3}, \Delta=\sum \Delta^{\prime} \otimes \Delta^{\prime \prime}$ and

$$
A:=\{(\chi, \mu, \mu),(\mu, \chi, \mu),(\mu, \mu, \chi)\}
$$

We hope that the meaning of this last notation is clear. For example, if $\left(\bigcirc_{1}, \bigcirc_{2}, \bigcirc_{3}\right)=(\chi, \mu, \mu)$, then

$$
\begin{aligned}
\left(\bigcirc_{1}\right. & \left.\otimes \bigcirc_{2} \otimes \bigcirc_{3}\right)((\mathbb{1} \otimes \Delta) \Delta(v) \bar{\otimes} \Phi) \\
& =\bigcirc_{1}\left(\Delta^{\prime}(v), \Phi_{1}\right) \otimes \bigcirc_{2}\left(\Delta^{\prime}\left(\Delta^{\prime \prime}(v)\right), \Phi_{2}\right) \otimes \bigcirc_{3}\left(\Delta^{\prime \prime}\left(\Delta^{\prime \prime}(v)\right), \Phi_{3}\right) \\
& =\chi\left(\Delta^{\prime}(v), \Phi_{1}\right) \otimes \Delta^{\prime}\left(\Delta^{\prime \prime}(v)\right) \cdot \Phi_{2} \otimes \Delta^{\prime \prime}\left(\Delta^{\prime \prime}(v)\right) \cdot \Phi_{3} .
\end{aligned}
$$

Equation (35) is the total variational derivative of quasi-coassociativity relative to all three components of the infinitesimal deformation. Its vanishing is the condition for preserving quasi-coassociativity under an infinitesimal deformation.

The map $d_{1}: \operatorname{Hom}_{\mathbf{k}}\left(\bar{V}, \otimes^{2} V\right) \rightarrow \operatorname{Hom}_{\mathbf{k}}\left(\mathbf{k}, \otimes^{4} V\right)$ describes the variational derivative of the pentagon identity relative to an infinitesimal variation in comultiplication $D$,

$$
\begin{aligned}
d_{1}(D)= & -\left(\mathbb{1}^{2} \otimes \Delta\right)(\Phi) \cdot\left(D \otimes \mathbb{1}^{2}\right)(\Phi)+(1 \otimes \Phi) \cdot(\mathbb{1} \otimes D \otimes \mathbb{1})(\Phi) \cdot(1 \otimes \Phi) \\
& -\left(\mathbb{1}^{2} \otimes D\right)(\Phi) \cdot\left(\Delta \otimes \mathbb{1}^{2}\right)(\Phi),
\end{aligned}
$$

for $D \in \operatorname{Hom}_{\mathbf{k}}\left(\bar{V}, \otimes^{2} V\right)$.

The variational derivative of the associativity operator relative to an infinitesimal automorphism, $\phi$, is given by the operator $d_{1}: \operatorname{Hom}_{\mathbf{k}}(\bar{V}, V) \rightarrow \operatorname{Hom}_{\mathbf{k}}\left(\mathbf{k}, \bigotimes^{3} V\right)$,

$$
d_{1}(\phi)=\left(\phi \otimes \mathbb{1}^{\otimes 2}\right)(\Phi)+(\mathbb{1} \otimes \phi \otimes \mathbb{1})(\Phi)+\left(\mathbb{1}^{\otimes 2} \otimes \phi\right)(\Phi),
$$




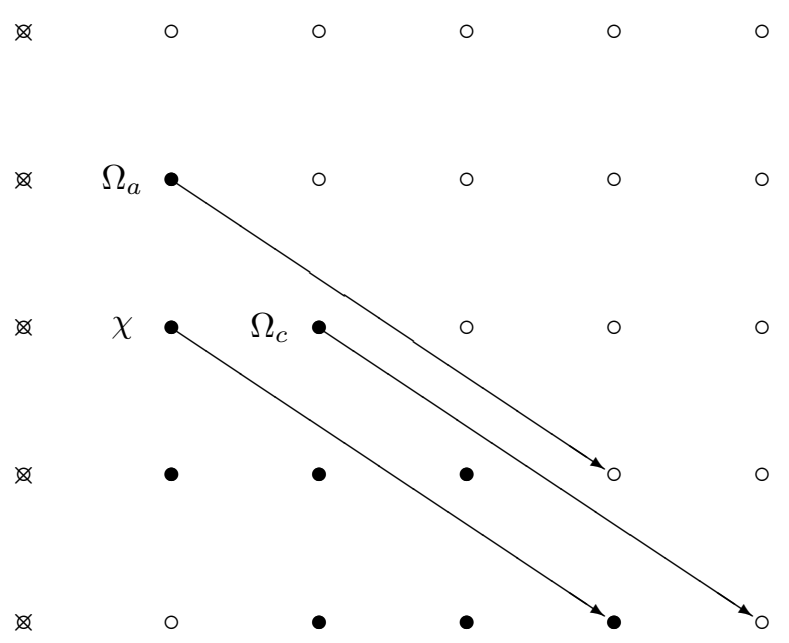

FIGURE 12. All the occurrences of the operator $d_{2}$ in deformation theory.

for $\phi \in \operatorname{Hom}_{\mathbf{k}}(\bar{V}, V)$. Together with $d_{\mathrm{q}-\mathrm{Car}}(F)$ this is the infinitesimal variation in $\Phi$ due to an infinitesimal twisting and an infinitesimal automorphism. In order to complete the discussion of (36) we need to compute $d_{2}(\chi)$, see Figure 12.

The variational derivative of the pentagon identity relative to an infinitesimal variation in multiplication, $\chi$, is described by the map $d_{2}: \operatorname{Hom}_{\mathbf{k}}\left(\otimes^{2} \bar{V}, V\right) \rightarrow$ $\mathrm{Hom}_{\mathbf{k}}\left(\mathbf{k}, \otimes^{4} V\right)$

$$
\begin{aligned}
& d_{2}(\chi)=\sum_{B}\left\{-\sum \bigcirc_{1}\left(\Phi_{1}, \Delta^{\prime}\left(\Phi_{1}\right)\right) \otimes \bigcirc_{2}\left(\Phi_{2}, \Delta^{\prime \prime}\left(\Phi_{1}\right)\right)\right. \\
& \otimes \bigcirc_{3}\left(\Delta^{\prime}\left(\Phi_{3}\right), \Phi_{2}\right) \otimes \bigcirc_{4}\left(\Delta^{\prime \prime}\left(\Phi_{3}\right), \Phi_{3}\right) \\
& +\sum \bigcirc_{1}\left(1, \Phi_{1} \cdot \Phi_{1}\right) \otimes \bigcirc_{2}\left(\Phi_{1}, \Delta^{\prime}\left(\Phi_{2}\right) \cdot \Phi_{2}\right) \\
& \otimes \bigcirc_{3}\left(\Phi_{2}, \Delta^{\prime \prime}\left(\Phi_{2}\right) \cdot \Phi_{3}\right) \otimes \bigcirc_{4}\left(\Phi_{3}, \Phi_{3}\right) \\
& +\sum(1 \otimes \Phi) \cdot\left[\bigcirc_{1}\left(\Phi_{1}, \Phi_{1}\right) \otimes \bigcirc_{2}\left(\Delta^{\prime}\left(\Phi_{2}\right), \Phi_{2}\right)\right. \\
& \left.\left.\otimes \bigcirc_{3}\left(\Delta^{\prime \prime}\left(\Phi_{2}\right), \Phi_{3}\right) \otimes \bigcirc_{4}\left(\Phi_{3}, 1\right)\right]\right\},
\end{aligned}
$$

for $\chi \in \operatorname{Hom}_{\mathbf{k}}\left(\otimes^{2} \bar{V}, V\right)$ and $\Delta=\sum \Delta^{\prime} \otimes \Delta^{\prime \prime}$, where the "big" summation is taken over

$$
\left(\bigcirc_{1}, \bigcirc_{2}, \bigcirc_{3}, \bigcirc_{4}\right) \in B:=\{(\chi, \mu, \mu, \mu),(\mu, \chi, \mu, \mu),(\mu, \mu, \chi, \mu),(\mu, \mu, \mu, \chi)\} .
$$

The elements of $A^{\otimes 4}$ whose components appear in the previous expression correspond to the associativity operators corresponding to the edges in the triangulation of the pentagon $K_{4}$ illustrated in Figure 7 with the first, second and third summands corresponding to the top, middle and bottom triangles respectively. Note that the sequence of transition operators read from left to right is the opposite of 


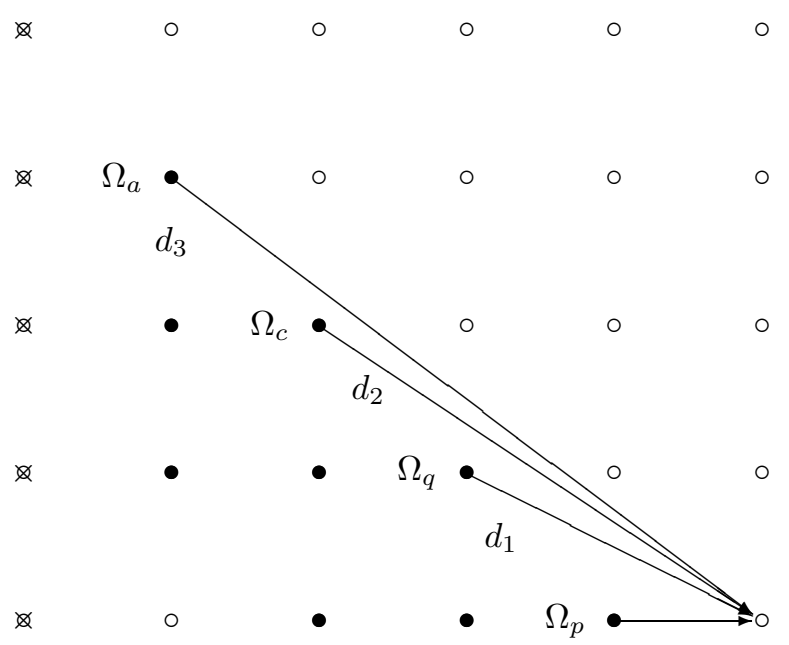

FIGURE 13. The one occurrence of $d_{3}$ in deformation cohomology.

their order with respect to the ordering of the vertices. This is a simple consequence of the usual rules for writing functional composition. The negative sign in the first summand comes from the fact that the orientation given by the ordering of the vertices is the reverse of the natural orientation in the boundary of the pentagon.

We see that (36) expresses the total variational derivative of the pentagon identity relative to all three components of the infinitesimal deformation. This finishes the proof that $\hat{H}^{2}(A)$ describes the infinitesimal deformations modulo infinitesimal automorphisms and twistings.

The proof that $\hat{H}^{3}(A)$ describes the obstructions to the integrability of an infinitesimal deformation, involves the first and only appearance of the operator $d_{3}$. It acts on precisely one component of the obstruction class, $\Omega_{a}$, the obstruction to associativity appearing in position $(1,3)$. For the full proof that the obstruction class, consisting of four components $\Omega_{a}, \Omega_{c}, \Omega_{q}$ and $\Omega_{p}$ as in Figure 13, is a cocycle we may either use the classical approach of [14], or the deviation calculus of [13]. This straightforward computation would stretch the length of the paper beyond any reasonable limit and is therefore omitted.

In the deformation theory, $d_{3}\left(\Omega_{a}\right)$ is one of the terms in the coboundary of the obstruction class, see Figure 13. We include the explicit formula even though it is very complicated since it is the first really non-trivial example of the general construction. We will write $d_{3}\left(\Omega_{a}\right)$ as

$$
\begin{aligned}
d_{3}\left(\Omega_{a}\right)= & M\left[\left(K_{2} \times K_{4}\right)_{4}\right]+M\left[\left(K_{2} \times K_{4}\right)_{3}\right]+M\left[\left(K_{2} \times K_{4}\right)_{2}\right] \\
& +M\left[\left(K_{4} \times K_{2}\right)_{2}\right]+M\left[\left(K_{3} \times K_{3}\right)_{2}\right] .
\end{aligned}
$$

Each term is a summation over all $\left(\bigcirc_{1}, \bigcirc_{2}, \bigcirc_{3}, \bigcirc_{4}, \bigcirc_{5}\right) \in B$, where

$$
\begin{aligned}
B:=\{ & \left(\Omega_{a}, \mu, \mu, \mu, \mu\right),\left(\mu, \Omega_{a}, \mu, \mu, \mu\right), \\
& \left.\left(\mu, \mu, \Omega_{a}, \mu, \mu\right),\left(\mu, \mu, \mu, \Omega_{a}, \mu\right),\left(\mu, \mu, \mu, \mu, \Omega_{a}\right)\right\} .
\end{aligned}
$$


The terms are:

$$
\begin{aligned}
& M\left[\left(K_{2} \times K_{4}\right)_{4}\right] \\
& =-\sum\left(\bigcirc_{1} \otimes \cdots \otimes \bigcirc_{5}\right)\left(\left(\mathbb{1}^{2} \otimes(\mathbb{1} \otimes \Delta) \Delta\right)(\Phi) \bar{\otimes}(\Delta \otimes \mathbb{1} \otimes \Delta)(\Phi)\right. \\
& \left.\bar{\otimes}\left((\Delta \otimes \mathbb{1}) \Delta \otimes \mathbb{1}^{2}\right)(\Phi)\right) \\
& +\sum\left(\bigcirc_{1} \otimes \cdots \otimes \bigcirc_{5}\right)\left(\left(1 \otimes\left(\mathbb{1}^{2} \otimes \Delta\right)(\Phi)\right) \bar{\otimes}\left(\left(\mathbb{1} \otimes \Delta^{2}\right)(\Phi) \cdot\left(\Phi \otimes 1^{2}\right)\right)\right. \\
& \left.\bar{\otimes}\left((\Delta \otimes \mathbb{1}) \Delta \otimes \mathbb{1}^{2}\right)(\Phi)\right) \\
& +\left(1 \otimes\left(\mathbb{1}^{2} \otimes \Delta\right)(\Phi)\right) \cdot \sum\left(\bigcirc_{1} \otimes \cdots \otimes \bigcirc_{5}\right)\left(\left(\mathbb{1} \otimes \Delta^{2}\right)(\Phi) \bar{\otimes}\left(\Phi \otimes 1^{2}\right)\right. \\
& \left.\bar{\otimes}\left((\Delta \otimes \mathbb{1}) \Delta \otimes \mathbb{1}^{2}\right)(\Phi)\right),
\end{aligned}
$$

$$
\begin{aligned}
& M\left[\left(K_{2} \times K_{4}\right)_{3}\right] \\
& =+\left(1^{2} \otimes \Phi\right) \cdot \sum\left(\bigcirc_{1} \otimes \cdots \otimes \bigcirc_{5}\right)\left(\left(\mathbb{1}^{2} \otimes(\Delta \otimes \mathbb{1}) \Delta\right)(\Phi) \bar{\otimes}\left(\left(\Delta^{2} \otimes \mathbb{1}\right) \Phi\right)\right. \\
& \left.\bar{\otimes}\left(\left(\Delta \otimes \mathbb{1}^{2}\right)(\Phi) \otimes 1\right)\right) \\
& -\left(1^{2} \otimes \Phi\right) \cdot \sum\left(\bigcirc_{1} \otimes \cdots \otimes \bigcirc_{5}\right)((1 \otimes(\mathbb{1} \otimes \Delta \otimes \mathbb{1})(\Phi)) \bar{\otimes}(\mathbb{1} \otimes(\mathbb{1} \otimes \Delta) \Delta \otimes \mathbb{1})(\Phi) \\
& \left.\cdot\left(\left(\mathbb{1}^{2} \otimes \Delta\right)(\Phi) \otimes 1\right) \bar{\otimes}\left(\left(\Delta \otimes \mathbb{1}^{2}\right)(\Phi) \otimes 1\right)\right) \\
& -\left(1^{2} \otimes \Phi\right) \cdot(1 \otimes(\mathbb{1} \otimes \Delta \otimes \mathbb{1})(\Phi)) \cdot \sum\left(\bigcirc_{1} \otimes \cdots \otimes \bigcirc_{5}\right)((\mathbb{1} \otimes(\mathbb{1} \otimes \Delta) \Delta \otimes \mathbb{1})(\Phi) \\
& \left.\bar{\otimes}\left(\left(\mathbb{1}^{2} \otimes \Delta\right)(\Phi) \otimes 1\right) \bar{\otimes}\left(\left(\Delta \otimes \mathbb{1}^{2}\right)(\Phi) \otimes 1\right)\right) \\
& M\left[\left(K_{2} \times K_{4}\right)_{2}\right] \\
& =-\left(1 \otimes\left(\mathbb{1}^{2} \otimes \Delta\right)(\Phi)\right) \cdot \sum\left(\bigcirc_{1} \otimes \cdots \otimes \bigcirc_{5}\right)\left(\left(\mathbb{1} \otimes \Delta^{2}\right)(\Phi)\right. \\
& \left.\bar{\otimes}\left((\mathbb{1} \otimes \Delta) \Delta \otimes \mathbb{1}^{2}\right)(\Phi) \bar{\otimes}\left(\Phi \otimes 1^{2}\right)\right) \\
& +\left(1 \otimes\left(\mathbb{1}^{2} \otimes \Delta\right)(\Phi)\right) \cdot \sum\left(\bigcirc_{1} \otimes \cdots \otimes \bigcirc_{5}\right)\left(\left(1 \otimes\left(\Delta \otimes \mathbb{1}^{2}\right)(\Phi)\right) \bar{\otimes}(\mathbb{1} \otimes(\Delta \otimes \mathbb{1}) \Delta \otimes \mathbb{1})(\Phi)\right. \\
& \left.\cdot((\mathbb{1} \otimes \Delta \otimes \mathbb{1})(\Phi) \otimes 1) \bar{\otimes}\left(\Phi \otimes 1^{2}\right)\right) \\
& +\left(1 \otimes\left(\mathbb{1}^{2} \otimes \Delta\right)(\Phi)\right) \cdot\left(1 \otimes\left(\Delta \otimes \mathbb{1}^{2}\right)(\Phi)\right) \cdot \sum\left(\bigcirc_{1} \otimes \cdots \otimes \bigcirc_{5}\right)((\mathbb{1} \otimes(\Delta \otimes \mathbb{1}) \Delta \otimes \mathbb{1})(\Phi) \\
& \left.\bar{\otimes}((\mathbb{1} \otimes \Delta \otimes \mathbb{1})(\Phi) \otimes 1) \bar{\otimes}\left(\Phi \otimes 1^{2}\right)\right) \\
& M\left[\left(K_{4} \times K_{2}\right)_{2}\right] \\
& =-\sum\left(\bigcirc_{1} \otimes \cdots \otimes \bigcirc_{5}\right)\left(\left(1 \otimes\left(\mathbb{1}^{2} \otimes \Delta\right)(\Phi)\right) \bar{\otimes}\left(1 \otimes\left(\Delta \otimes \mathbb{1}^{2}\right)(\Phi)\right)\right. \\
& \left.\bar{\otimes}(\mathbb{1} \otimes(\Delta \otimes \mathbb{1}) \Delta \otimes \mathbb{1})(\Phi) \cdot((\mathbb{1} \otimes \Delta \otimes \mathbb{1})(\Phi) \otimes 1) \cdot\left(\Phi \otimes 1^{2}\right)\right) \\
& +\left(1^{2} \otimes \Phi\right) \cdot \sum\left(\bigcirc_{1} \otimes \cdots \otimes \bigcirc_{5}\right)((1 \otimes(\mathbb{1} \otimes \Delta \otimes \mathbb{1})(\Phi)) \bar{\otimes}(1 \otimes \Phi \otimes 1) \\
& \left.\bar{\otimes}(\mathbb{1} \otimes(\Delta \otimes \mathbb{1}) \Delta \otimes \mathbb{1})(\Phi) \cdot((\mathbb{1} \otimes \Delta \otimes \mathbb{1})(\Phi) \otimes 1) \cdot\left(\Phi \otimes 1^{2}\right)\right) \\
& +\sum\left(\bigcirc_{1} \otimes \cdots \otimes \bigcirc_{5}\right)\left(\left(1^{2} \otimes \Phi\right) \bar{\otimes}(1 \otimes(\mathbb{1} \otimes \Delta \otimes \mathbb{1})(\Phi)) \cdot(1 \otimes \Phi \otimes 1)\right. \\
& \left.\bar{\otimes}(\mathbb{1} \otimes(\Delta \otimes \mathbb{1}) \Delta \otimes \mathbb{1})(\Phi) \cdot((\mathbb{1} \otimes \Delta \otimes \mathbb{1})(\Phi) \otimes 1) \cdot\left(\Phi \otimes 1^{2}\right)\right) \\
& M\left[\left(K_{3} \times K_{3}\right)_{2}\right] \\
& =-\left(1^{2} \otimes \Phi\right) \cdot(1 \otimes(\mathbb{1} \otimes \Delta \otimes \mathbb{1})(\Phi)) \cdot \sum\left(\bigcirc_{1} \otimes \cdots \otimes \bigcirc_{5}\right)((1 \otimes \Phi \otimes 1) \\
& \bar{\otimes}(\mathbb{1} \otimes(\Delta \otimes \mathbb{1}) \Delta \otimes \mathbb{1})(\Phi) \\
& \left.\bar{\otimes}((\mathbb{1} \otimes \Delta \otimes \mathbb{1})(\Phi) \otimes 1) \cdot\left(\Phi \otimes 1^{2}\right)\right) \\
& +\left(1^{2} \otimes \Phi\right) \cdot(1 \otimes(\mathbb{1} \otimes \Delta \otimes \mathbb{1})(\Phi)) \cdot \sum\left(\bigcirc_{1} \otimes \cdots \otimes \bigcirc_{5}\right)((\mathbb{1} \otimes(\mathbb{1} \otimes \Delta) \Delta \otimes \mathbb{1})(\Phi) \\
& \left.\bar{\otimes}(1 \otimes \Phi \otimes 1) \bar{\otimes}((\mathbb{1} \otimes \Delta \otimes \mathbb{1})(\Phi) \otimes 1) \cdot\left(\Phi \otimes 1^{2}\right)\right)
\end{aligned}
$$


and, finally

$$
\begin{gathered}
M\left[\left(K_{3} \times K_{3}\right)_{3}\right]=-\sum\left(\bigcirc_{1} \otimes \cdots \otimes \bigcirc_{5}\right)\left(\left(1^{2} \otimes \Phi\right) \bar{\otimes}\left(\mathbb{1}^{2} \otimes(\Delta \otimes \mathbb{1}) \Delta\right)(\Phi)\right. \\
\left.\bar{\otimes}\left(\left(\Delta^{2} \otimes \mathbb{1}\right) \Phi\right) \cdot\left(\left(\Delta \otimes \mathbb{1}^{2}\right)(\Phi) \otimes 1\right)\right) \\
+\sum\left(\bigcirc_{1} \otimes \cdots \otimes \bigcirc_{5}\right)\left(\left(\mathbb{1}^{2} \otimes(\mathbb{1} \otimes \Delta) \Delta\right)(\Phi)\right. \\
\left.\bar{\otimes}\left(1^{2} \otimes \Phi\right) \bar{\otimes}\left(\left(\Delta^{2} \otimes \mathbb{1}\right) \Phi\right) \cdot\left(\left(\Delta \otimes \mathbb{1}^{2}\right)(\Phi) \otimes 1\right)\right) .
\end{gathered}
$$

On the one hand these terms can be understood in terms of the edges of the simplices in $\mathcal{T}_{5}$, our canonical triangulation of $K_{5}$. Recall that the triangulation is induced by the cone over the triangulations of $\left(K_{a} \times K_{b}\right)_{c}$ for $a+b=6$ and $2 \leq c \leq b$. The bracketed expression on the left side of these equations indicates the part of the boundary of $K_{5}$ whose cone determines the components on the right. Figures 14 and 15 illustrate the structure of $K_{5}$, and we list explicitly the 3 -simplices in $\tilde{\mathcal{T}}_{5}$ in decreasing order of the vertices to be consistent with the order in which the transition operators appear:

$$
\begin{array}{lll}
\operatorname{Cone}\left(\left(K_{2} \times K_{4}\right)_{4}, \xi_{5}\right) & : & {[1,2,13,14],[1,8,13,14],[8,11,13,14]} \\
\operatorname{Cone}\left(\left(K_{2} \times K_{4}\right)_{3}, \xi_{5}\right) & : & {[3,4,7,14],[3,5,7,14],[5,6,7,14]} \\
\operatorname{Cone}\left(\left(K_{2} \times K_{4}\right)_{2}, \xi_{5}\right) & : & {[8,11,12,14],[8,9,12,14],[9,10,12,14]} \\
\operatorname{Cone}\left(\left(K_{4} \times K_{2}\right)_{2}, \xi_{5}\right) & : & {[1,8,9,14],[3,5,9,14],[1,3,9,14]} \\
\operatorname{Cone}\left(\left(K_{3} \times K_{3}\right)_{2}, \xi_{5}\right) & : & {[5,9,10,14],[5,6,10,14]} \\
\operatorname{Cone}\left(\left(K_{3} \times K_{3}\right)_{3}, \xi_{5}\right) & : & {[1,3,4,14],[1,2,4,14]}
\end{array}
$$

On the other hand we can understand the terms as arising from the substitution of $\Omega_{a}$ wherever the associativity of multiplication is assumed in the original proof of $d_{\mathrm{q}-\mathrm{Car}}\left(\Omega_{p}\right)=0$, the cocycle condition for restricted deformations where multiplication and comultiplication are not deformed, as given in [14] or using the deviation calculus of [13]. We can distinguish the summands in terms of the part of $d_{\mathrm{q}-\mathrm{Car}}\left(\Omega_{p}\right)$ in which we want to use associativity. The first set of three summands, $M\left[\left(K_{2} \times K_{4}\right)_{4}\right]$, in our list comes from the part of $d_{\mathrm{q}-\mathrm{Car}}\left(\Omega_{p}\right)$ involving

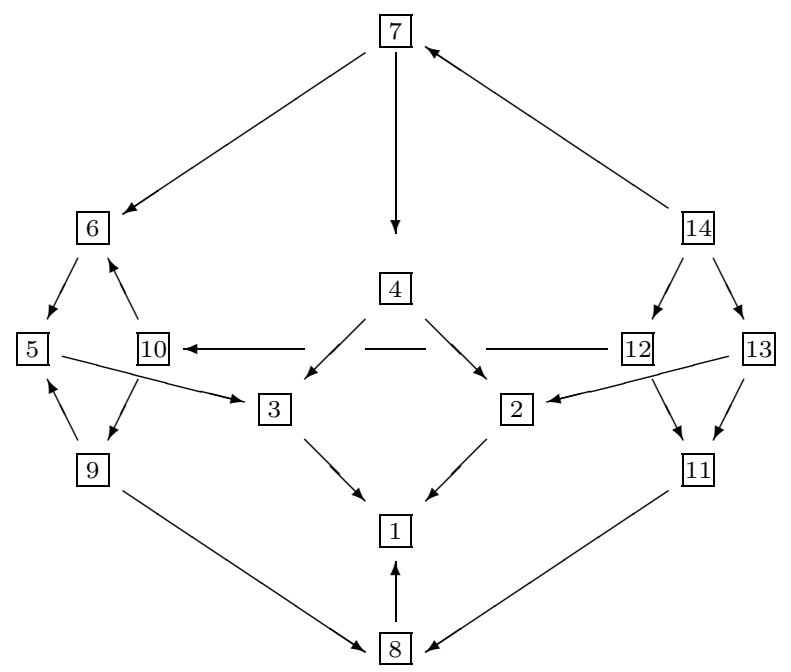

FIGURE $14 . \quad K_{5}$ as a cell in three dimensions. 


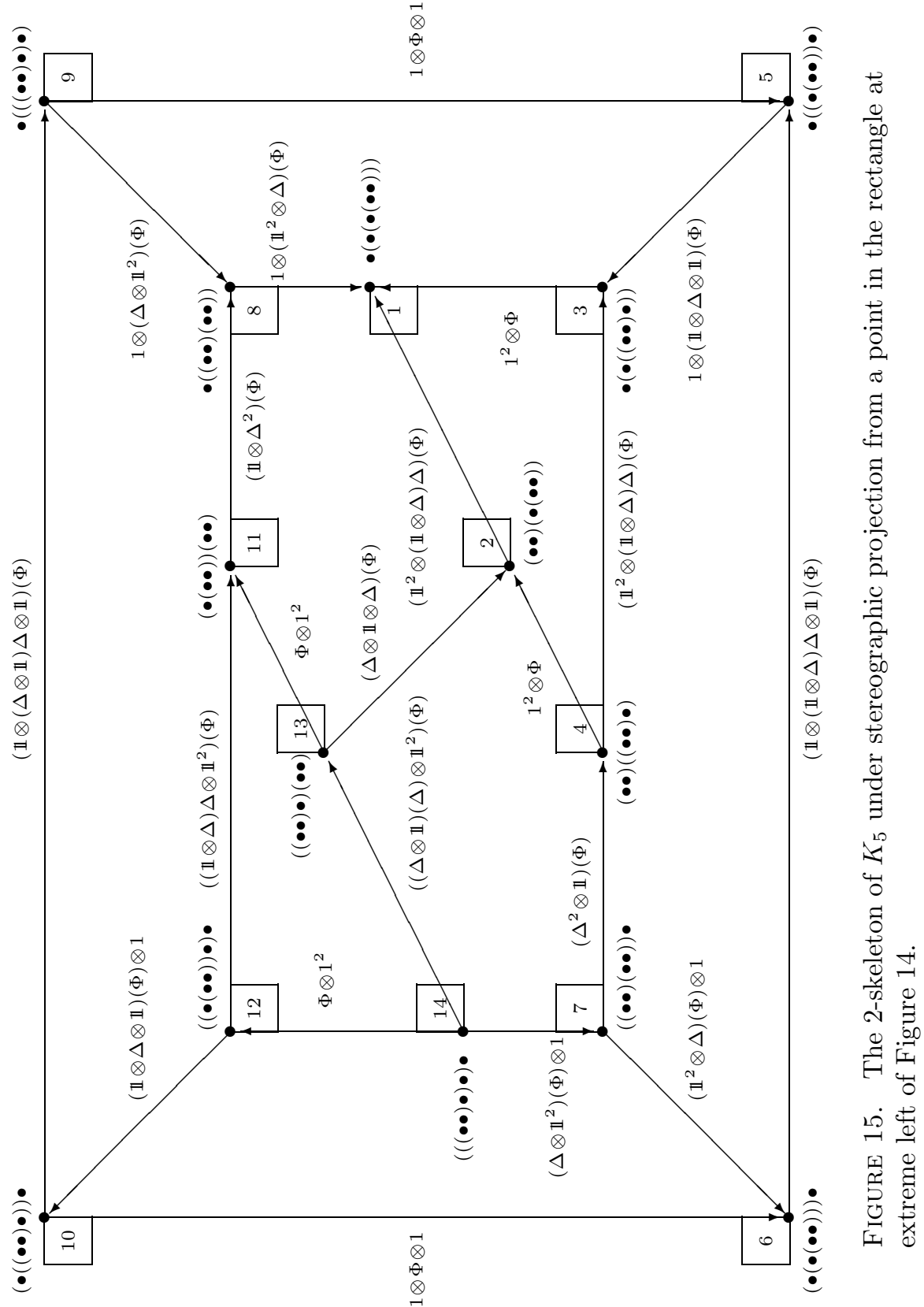


$\left(\mathbb{1}^{3} \otimes \Delta\right)\left(\Omega_{p}\right)$, the second set of three terms comes from the part involving $\left(\mathbb{1}^{2} \otimes\right.$ $\Delta \otimes \mathbb{1})\left(\Omega_{p}\right)$, the third set comes from $\left(\mathbb{1} \otimes \Delta \otimes \mathbb{1}^{2}\right)\left(\Omega_{p}\right)$ etc.

Before ending this section we describe an application to the deformation theory of Drinfel'd's quasi-Hopf quantization $A_{h}=\left(U(\mathcal{G})[[h]], \cdot, \Delta, \Phi_{h}\right)$ of the universal enveloping algebra of a simple Lie algebra $\mathcal{G}$ over the rational field, $\mathbf{k}=\mathbf{Q}$. Here $A_{h}$ is as in [15, Chapter 11], i.e. · and $\Delta$ are undeformed and $\Phi_{h}=1+$ a polynomial in $h^{2}$. Let us prove first the following lemma.

Lemma 6.3. The second cohomology group $\hat{H}^{2}\left(A_{0}\right)$ of the undeformed algebra $A_{0}$ is trivial. The third cohomology group $\hat{H}^{3}\left(A_{0}\right)$ has dimension one and is generated by the infinitesimal of the deformation $A_{h}$.

Proof. As we have already observed, the cohomology $\hat{H}^{n}\left(A_{0}\right)$ for the undeformed algebra $A_{0}=\left(U(\mathcal{G}), \cdot, \Delta, \Phi_{0}=1\right)$ is the same as the cohomology of the extended bicomplex constructed by M. Gerstenhaber and D. Schack for the bialgebra cohomology, see also [15, Chapter 11] for details. There are no invariants in $\wedge^{2} \mathcal{G}$ and the invariants in $\wedge^{3} \mathcal{G}$ form a one-dimensional subspace spanned by the infinitesimal of $A_{h}$ (see again [15, Chapter 11]). These facts together with an easy spectral sequence argument give the desired result.

Proposition 6.4. $\hat{H}^{2}\left(A_{h}\right)=0$ and $\hat{H}^{3}\left(A_{h}\right) \cong \hat{H}^{3}\left(A_{0}\right) \otimes \mathbf{Q}[[h]]$.

Proof. Standard formal power series arguments (see [7, par. 7] and the discussion below) prove that the same semicontinuity principle holds for the cohomology of a deformation of a Drinfel'd algebra as holds for the deformation of an associative algebra [7, par. 7]. The first part is an easy consequence of this fact and the triviality of $\hat{H}^{2}\left(A_{0}\right)$ (Lemma 6.3).

To compute $\hat{H}^{3}\left(A_{h}\right)$ we can use the spectral sequence associated to the filtration

$$
F^{p}\left(\hat{C}\left(A_{h}\right)\right)=\bigoplus_{i \geq p} E^{i, j}\left(A_{h}\right)
$$

The first term of the spectral sequence is

$$
E_{1}^{i, j}=\operatorname{Hoch}^{j}\left(U(\mathcal{G}), U(\mathcal{G})^{\otimes i}\right) \otimes \mathbf{Q}[[h]]=\mathrm{H}_{C E}^{j}(\mathcal{G}) \otimes\left(U(\mathcal{G})^{\otimes i}\right)^{\mathcal{G}} \otimes \mathbf{Q}[[h]],
$$

where $\operatorname{Hoch}(U(\mathcal{G}), M)$ is the Hochschild cohomology of $U(\mathcal{G})$ with coefficients in $M$, $\mathrm{H}_{C E}(\mathcal{G})$ is the Chevalley-Eilenberg cohomology with trivial coefficients, and $M^{\mathcal{G}}$ is the space of $\mathcal{G}$ invariants. Since $\mathrm{H}_{C E}^{j}(\mathcal{G})=0$ for $j=1,2$, and we must have $i \geq 1$, the component of $E_{1}$ in dimension $i+j-1 \leq 2$ is $\left.\left(U(\mathcal{G})^{\otimes i}\right)\right)^{\mathcal{G}}[[h]]$. The coboundary operator on $E_{1}$, is $d_{1}=d_{\mathrm{q}-\mathrm{Car}}$.

All the higher coboundary operators, $d_{n}$, in the spectral sequence are zero in the relevant range. Therefore, to conclude the proof of the proposition, it is enough to show that $H^{3}\left(E_{1}, d_{1}\right)$ is a free rank one module over $\mathbf{Q}[[h]]$. The same standard methods for studying the cohomology of a deformed algebra, which we mentioned above in relation to semi-continuity can be applied here as well. The only difference is that the differential is not standard.

For the purpose of completeness we present the full proof of the following lemma.

Lemma 6.5. The cohomology $H^{3}\left(E_{1}, d_{1}\right)$ is a free $\mathbf{Q}[[h]]$-module of rank one generated by $\psi_{h}=\frac{1}{h} \frac{d}{d h} \Phi_{h}$.

Proof. The coboundary operator $d_{\mathrm{q}-\mathrm{Car}}$ has an expansion in even powers of $h$,

$$
d_{\mathrm{q}-\mathrm{Car}}=d_{h}=\sum d_{2 n} h^{2 n}
$$


with $d_{0}=d_{\text {Car }}$ the standard Cartier coboundary. As we have seen above, the $d_{\mathrm{q}-\mathrm{Car}} \Psi$ is, up to conjugation, the derivative $\frac{d}{d t}$ of the left side of the pentagon identity, $\operatorname{Pent}\left(\Phi_{h}+t \Psi\right)=0$. Therefore differentiating the pentagon identity for $\Phi_{h}$ relative to $h$, using the chain rule, gives $d_{h} \frac{d}{d h} \Phi_{h}=0$. Since $\Phi_{h}$ is a formal power series in $h^{2}$, we can divide by $h$ and conclude that $\psi_{h}$ is a $d_{h}$ cocycle. We claim that any $d_{h} 3$-cocycle is cohomologous to a $\mathbf{Q}[[h]]$ multiple of $\psi_{h}$. Let $\sigma_{h}$ be such a 3 -cocycle. We will prove that there exist a formal power series $c_{h}$ and a 2-cochain $\rho_{h}$ such that $\sigma_{h}=c_{h} \psi_{h}+d_{h} \rho_{h}$. The proof is by induction. Consider the following truncations at $h^{n+1}$ : let $d^{n}$ be the truncation of $d_{h}, \psi^{n}$, the truncation of $\psi_{h}$, and $\sigma^{n}$, the truncation of $\sigma_{h}$. Assume that there exist partial series $c^{n}=c_{0}+\cdots+c_{n} h^{n}$ and $\rho^{n}=\rho_{0}+\cdots+\rho_{n} h^{n}$ such that

$$
\sigma^{n}=c^{n} \psi^{n}+d^{n} \rho^{n} \quad \bmod \quad h^{n+1} .
$$

Define $\xi_{n+1}$ by

$$
\xi_{n+1} h^{n+1}=\sigma^{n+1}-\left(c^{n} \psi^{n+1}+d^{n+1} \rho^{n}\right) \bmod h^{n+2} .
$$

Then

$$
d_{0} \xi_{n+1} h^{n+1}=d^{n+1}\left(\sigma^{n+1}-c^{n} \psi^{n+1}-d^{n+1} \rho^{n}\right)=0 \quad \bmod \quad h^{n+2}
$$

since $d^{n+1} \sigma^{n+1}=d^{n+1} \psi^{n+1}=d^{n+1} d^{n+1} \rho^{n}=0 \bmod h^{n+2}$. Thus $\xi_{n+1}$ is a $d_{0}$ cocycle. Now $\phi=\psi_{0}$ generates the $\mathcal{G}$-invariant $d_{0}$-cohomology so there exist a constant $c_{n+1}$ and a 2-cochain $\rho_{n+1}$ such that

$$
\xi_{n+1}=c_{n+1} \psi_{0}+d_{0} \rho_{n+1} \text {. }
$$

Define $\rho^{n+1}=\rho^{n}+\rho_{n+1} h^{n+1}$ and $c^{n+1}=c^{n}+c_{n+1} h^{n+1}$. Then

$$
\sigma^{n+1}=c^{n+1} \psi^{n+1}+d^{n+1} \rho^{n+1} \bmod h^{n+2},
$$

completing the induction.

This shows that the cohomology group under consideration is generated by $\psi_{h}$. Next we must show that it is torsion-free as a $\mathbf{Q}[[h]]$-module. Suppose that there exists an exponent $n$ such that

$$
h^{n} \psi_{h}=d_{h} \xi_{h}
$$

and consider the minimal such exponent. Then $\xi_{h}$ has $\xi_{0} \neq 0$, that is, nonvanishing constant $(h=0)$ term. We know that $n>0$ since $\psi_{0}=\phi$ is not a $d_{0}$-coboundary. Thus $\xi_{0}$ is a cocycle and since $H^{2}\left(A, d_{0}\right)=0$, we can find a $\chi_{h} \in C^{1}$ such that $\xi_{h}=d_{h} \chi_{h} \bmod h$. Therefore

$$
d_{h}\left(\xi_{h}-d_{h} \chi_{h}\right)=d_{h} \xi_{h}=h^{n} \psi_{h} .
$$

The term in parentheses is divisible by $h$ so we can reduce the exponent $n$ by one, in contradiction to minimality. Thus the cohomology, which is generated by $\psi_{h}$, is a torsion-free module over $\mathbf{Q}[[h]]$ and since the latter is a local ring, any torsion-free module is free.

Definition 6.6. In formal deformation theory, a deformation is called a "jump deformation" if the generic two-parameter deformation $A_{h(1+u)}$ is equivalent to the deformation $A_{h}[[u]]$, which is trivial as a deformation in the parameter $u$ of $A_{h}$.

A necessary condition for a deformation to be a jump deformation is that the lifting of the infinitesimal be a coboundary in the cohomology of the deformed algebra. We have just shown the contrary so we have proved the following theorem. 
Theorem 6.7. The Drinfel'd deformation $A_{h}$ is not a jump deformation.

For the terminology and related analysis we refer the reader to $[7, \S 7$,$] and [4$, $\S 6]$. These papers work with the Hochschild cohomology, but their results apply to our situation as well. It follows from the general theory that the triviality of $\hat{H}^{2}\left(A_{0}\right)$ implies that there are no "jump deformations", but we have presented a more elementary argument.

\section{Proofs of the technical sublemmas}

We now prove the two technical sublemmas from Section 4.

Proof of Sublemma 4.9. Suppose we have some $(j, \pi) \in S_{a \Psi}^{\prime \prime}$, i.e. some $l, 1 \leq l \leq k$, such that $\pi(l)>\pi(l-1)$ and $0<j=\pi(l)+l-2$. We have, by definition,

$$
\begin{aligned}
& \left(\mathbb{1}^{\odot(r-1)} \odot d_{\mathcal{B}}^{j} \odot \mathbb{1}^{\odot(n-r)}\right) M_{\pi}^{r}(\hat{a}) \\
& \quad=\square_{v_{k}, v_{0}}\left(\sum \bar{\mu}\left(\psi_{1}\right) \otimes \cdots \otimes \bar{\mu}\left(\psi_{r-1}\right) \otimes \psi_{r} \otimes \bar{\mu}\left(\psi_{r+1}\right) \otimes \cdots \otimes \bar{\mu}\left(\psi_{n}\right)\right)
\end{aligned}
$$

where

$$
\psi_{i}=\sum a_{0}^{\left(v_{0}, i\right)} \otimes \cdots \otimes a_{\pi(l)-1}^{\left(v_{l-1}, i\right)} \cdot \Psi_{v_{l-1}, v_{l}}^{i} \otimes \cdots \otimes a_{q+1}^{\left(v_{k}, i\right)}, \quad 1 \leq i \leq n,
$$

while

$$
\begin{aligned}
& \left(\mathbb{1}^{\odot(r-1)} \odot d_{\mathcal{B}}^{j} \odot \mathbb{1}^{\odot(n-r)}\right) M_{\alpha(\pi)}^{r}(\hat{a}) \\
& \quad=\square_{v_{k}, v_{0}}\left(\sum \bar{\mu}\left(\psi_{1}^{\prime}\right) \otimes \cdots \otimes \bar{\mu}\left(\psi_{r-1}^{\prime}\right) \otimes \psi_{r}^{\prime} \otimes \bar{\mu}\left(\psi_{r+1}^{\prime}\right) \otimes \cdots \otimes \bar{\mu}\left(\psi_{n}^{\prime}\right)\right)
\end{aligned}
$$

with

$$
\psi_{i}^{\prime}=\sum a_{0}^{\left(v_{0}, i\right)} \otimes \cdots \otimes \Psi_{v_{l-1}, v_{l}}^{i} \cdot a_{\alpha(\pi)(l)}^{\left(v_{l}, i\right)} \otimes \cdots \otimes a_{q+1}^{\left(v_{k}, i\right)}, \quad 1 \leq i \leq n .
$$

But $\alpha(\pi)(l)=\pi(l)-1$ by definition and

$$
\sum \bigotimes_{i=1}^{n} a_{\pi(l)-1}^{\left(v_{l-1}, i\right)} \cdot \Psi_{v_{l-1}, v_{l}}^{i}=\sum \bigotimes_{i=1}^{n} \Psi_{v_{l-1}, v_{l}}^{i} \cdots a_{\pi(l)-1}^{\left(v_{l}, i\right)}
$$

by (19), and (25) follows "modulo the signs". We must show that $\operatorname{sgn}(\pi, \sigma)=$ $-\operatorname{sgn}(\alpha(\pi), \sigma)$ which is, by the definition of $\operatorname{sgn}(-,-)$, the same as $(-1)^{o(\pi)}=$ $-(-1)^{o(\alpha(\pi))}$, which follows from $o(\alpha(\pi))=o(\pi)-1$. Thus $(25)$ is proven.

Let $(j, \pi) \in T$, i.e. $0 \leq j \leq q$ and $\pi \in P(q-1, k)$. Then $M_{\pi}^{r}[\sigma]\left(d_{\mathcal{B}}^{j}(\hat{a})\right)=\square_{v_{k}, v_{0}}\left(\sum \bar{\mu}\left(\omega_{1}\right) \otimes \cdots \otimes \bar{\mu}\left(\omega_{r-1}\right) \otimes \omega_{r} \otimes \bar{\mu}\left(\omega_{r+1}\right) \otimes \cdots \otimes \bar{\mu}\left(\omega_{n}\right)\right)$,

where

$$
\begin{array}{rr}
\Omega_{i}=\sum a_{0}^{\left(v_{0}, i\right)} \otimes \cdots \otimes \Psi_{v_{l-1}, v_{l}}^{i} & \otimes \cdots \otimes\left(a_{j} \cdot a_{j+1}\right)^{\left(v_{l}, i\right)} \otimes \cdots \otimes \Psi_{v_{l}, v_{l+1}}^{i} \\
\otimes \cdots \otimes a v_{k} i q+1, & 1 \leq i \leq n,
\end{array}
$$

while

$$
\begin{aligned}
& \left(\mathbb{1}^{\odot(r-1)} \odot d_{\mathcal{B}}^{\beta(j)} \odot \mathbb{1}^{\odot(n-r)}\right) M_{\beta(\pi)}^{r}(\hat{a}) \\
& \quad=\square_{v_{k}, v_{0}}\left(\sum \bar{\mu}\left(\omega_{1}^{\prime}\right) \otimes \cdots \otimes \bar{\mu}\left(\omega_{r-1}^{\prime}\right) \otimes \omega_{r}^{\prime} \otimes \bar{\mu}\left(\omega_{r+1}^{\prime}\right) \otimes \cdots \otimes \bar{\mu}\left(\omega_{n}^{\prime}\right)\right),
\end{aligned}
$$

with

$$
\begin{array}{r}
\omega_{i}^{\prime}=\sum a_{0}^{\left(v_{0}, i\right)} \otimes \cdots \otimes \Psi_{v_{l-1}, v_{l}}^{i} \otimes \cdots \otimes a_{j}^{\left(v_{l}, i\right)} \cdot a_{j+1}^{\left(v_{l}, i\right)} \otimes \cdots \otimes \Psi_{v_{l}, v_{l+1}}^{i} \otimes \cdots \otimes a_{q+1}^{\left(v_{k}, i\right)}, \\
1 \leq i \leq n,
\end{array}
$$


and (26) "modulo the signs" follows from the fact that $\sum \bigotimes_{i=1}^{n}\left(a_{j} \cdot a_{j+1}\right)^{\left(v_{l}, i\right)}=$ $\sum \bigotimes_{i=1}^{n} a_{j}^{\left(v_{l}, i\right)} \cdot a_{j+1}^{\left(v_{l}, i\right)}$ (the multiplicativity of $\Delta$ ). It remains to show that

$$
(-1)^{n+\beta(j)} \cdot \operatorname{sgn}(\beta(\pi), \sigma)=(-1)^{k+n+j} \cdot \operatorname{sgn}(\pi, \sigma) .
$$

The desired relation reduces to congruence modulo 2

$$
\beta(j)+o(\beta(\pi)) \equiv j+k+o(\pi)
$$

which follows from $o(\beta(\pi))=o(\pi)+k-l$ and $\beta(j)=j+l$. The equation (26) is proven.

Let $\pi \in P(q, l-1)$. By definition,

$$
M_{\pi}^{r}\left[d_{S}^{0}(\sigma)\right]=\square_{v_{k}, v_{0}}\left(\sum \bar{\mu}\left(\nu_{1}\right) \otimes \cdots \otimes \bar{\mu}\left(\nu_{r-1}\right) \otimes \nu_{r} \otimes \bar{\mu}\left(\nu_{r+1}\right) \otimes \cdots \otimes \bar{\mu}\left(\nu_{n}\right)\right),
$$

where

$$
\nu_{i}=\sum a_{0}^{\left(v_{1}, i\right)} \otimes \cdots \otimes a_{\pi(1)-1}^{\left(v_{1}, i\right)} \otimes \Psi_{v_{1}, v_{2}}^{i} \otimes \cdots \otimes a_{q+1}^{\left(v_{k}, i\right)}, \quad 1 \leq i \leq n,
$$

while

$$
\begin{aligned}
& \left(\mathbb{1}^{\odot(r-1)} \odot d_{\mathcal{B}}^{0} \odot \mathbb{1}^{\odot(n-r)}\right) M_{\gamma(\pi)}^{r}(\hat{a}) \\
& \quad=\square_{v_{k}, v_{0}}\left(\sum \bar{\mu}\left(\nu_{1}^{\prime}\right) \otimes \cdots \otimes \bar{\mu}\left(\nu_{r-1}^{\prime}\right) \otimes \nu_{r}^{\prime} \otimes \bar{\mu}\left(\nu_{r+1}^{\prime}\right) \otimes \cdots \otimes \bar{\mu}\left(\nu_{n}^{\prime}\right)\right),
\end{aligned}
$$

with

$$
\nu_{i}^{\prime}=\sum a_{0}^{\left(v_{0}, i\right)} \cdot \Psi_{v_{0}, v_{1}}^{i} \otimes \cdots \otimes a_{\pi(1)-1}^{\left(v_{1}, i\right)} \Psi_{v_{1}, v_{2}}^{i} \otimes \cdots \otimes a_{q+1}^{\left(v_{k}, i\right)}, \quad 1 \leq i \leq n .
$$

From the equation $\sum \bigotimes_{i=1}^{n} a_{0}^{\left(v_{0}, i\right)} \cdot \Psi_{v_{0}, v_{1}}^{i}=\sum \bigotimes_{i=1}^{n} \Psi_{v_{0}, v_{1}}^{i} \cdot a_{0}^{\left(v_{1}, i\right)}$ (see (19)) we get that

$$
\begin{aligned}
& \sum \bar{\mu}\left(\nu_{1}^{\prime}\right) \otimes \cdots \otimes \bar{\mu}\left(\nu_{r-1}^{\prime}\right) \otimes \nu_{r}^{\prime} \otimes \bar{\mu}\left(\nu_{r+1}^{\prime}\right) \otimes \cdots \otimes \bar{\mu}\left(\nu_{n}^{\prime}\right) \\
& \quad=\Psi_{v_{0}, v_{1}} \bullet\left(\sum \bar{\mu}\left(\nu_{1}\right) \otimes \cdots \otimes \bar{\mu}\left(\nu_{r-1}\right) \otimes \nu_{r} \otimes \bar{\mu}\left(\nu_{r+1}\right) \otimes \cdots \otimes \bar{\mu}\left(\nu_{n}\right)\right)
\end{aligned}
$$

and (27) "modulo the signs" follows from Lemma 5.5. We must also verify that $\operatorname{sgn}\left(\pi, d_{S}^{0}(\sigma)\right)=(-1)^{n-1} \operatorname{sgn}(\gamma(\pi), \sigma)$. The verification reduces to checking that $(-1)^{o(\pi)+(k-1) n}=(-1)^{o(\gamma(\pi))+k n+n-1}$ which follows from $o(\pi)=o(\gamma(\pi))+1$. This finishes the proof of $(27)$.

The proof of (28) is a "mirror image" of the proof of (27), except that here the desired relation between the signs of the partitions is $(-1)^{k+o(\pi)+(k-1) n}=$ $(-1)^{n+k+q-1+o(\delta(\pi)+k n}$.

Proof of Sublemma 4.11. First, observe that $M_{\pi}^{r}\left[\sigma \diamond_{t, \phi} \tau\right](\hat{a})$ can be written as $M_{\pi}^{l}\left[\sigma \diamond_{t, \phi} \tau\right](\hat{a})$

$$
=\square_{\left(v_{r}, w_{s}\right)_{t},\left(v_{0}, w_{0}\right)_{t}}\left(\sum \bar{\mu}\left(\xi_{1}\right) \otimes \cdots \otimes \bar{\mu}\left(\xi_{l-1}\right) \otimes \xi_{l} \otimes \bar{\mu}\left(\xi_{l+1}\right) \otimes \cdots \otimes \bar{\mu}\left(\xi_{a+b-1}\right),\right.
$$

where, for $1 \leq d \leq a+b-1$,

$$
\begin{aligned}
\xi_{d}=\sum & a_{0}^{\left(\left(v_{\phi^{\prime}(0)}, w_{\phi^{\prime \prime}(0)}\right)_{t}, d\right)} \otimes \cdots \otimes a_{\pi(1)-1}^{\left(\left(v_{\phi^{\prime}(0)}, w_{\phi^{\prime \prime}(0)}\right)_{t}, d\right)} \\
& \otimes \Psi_{\left(v_{\phi^{\prime}(0)}, w_{\phi^{\prime \prime}(0)}\right)_{t},\left(v_{\phi^{\prime}(1)}, w_{\phi^{\prime \prime}(1)}\right)_{t}}^{d} \\
& \otimes \cdots \otimes \Psi_{\left(v_{\phi^{\prime}(r+s-1)}, w_{\phi^{\prime \prime}(r+s-1)}\right)_{t},\left(v_{\phi^{\prime}(r+s)}, w_{\phi^{\prime \prime}(r+s)}\right)_{t}}^{\left(\left(v_{\phi^{\prime}(r+s)}, w_{\phi^{\prime \prime}(r+s)}\right) t, d\right)} \\
& \otimes a_{\pi(r+s)}^{\left(\left(v_{\phi^{\prime}(r+s)}, w_{\phi^{\prime \prime}(r+s)}\right), d\right)} \otimes \cdots \otimes a_{q+1}^{(r)}
\end{aligned}
$$


Let us introduce the following terminology to distinguish those edges in $\sigma \diamond_{t, \phi} \tau$ along which the vertex from $\sigma$ changes and those in which the vertex from $\tau$ changes. We say that a shuffle $\phi \in \mathrm{Sh}_{r, s}$ has a jump of the 1st type (resp. of the 2nd type) at some point $h, 1 \leq h \leq r+s$, if $h \in\left\{i_{1}<\cdots<i_{r}\right\}$ (resp. $h \in\left\{j_{1}<\cdots<j_{s}\right\}$ ) or, equivalently, if $\phi^{\prime}(h)=\phi^{\prime}(h-1)+1$ and $\phi^{\prime \prime}(h)=\phi^{\prime \prime}(h-1)\left(\right.$ resp. $\phi^{\prime}(h)=\phi^{\prime}(h-1)$ and $\left.\phi^{\prime \prime}(h)=\phi^{\prime \prime}(h-1)+1\right)$.

It follows directly from the definitions that if $\phi$ has a jump of the 1st type at $h$, then

$$
\begin{aligned}
& \Psi_{\left(v_{\phi^{\prime}(h-1)}, w_{\phi^{\prime \prime}(h-1)}\right)_{t},\left(v_{\phi^{\prime}(h)}, w_{\phi^{\prime \prime}(h)}\right)_{t}} \\
& \quad=\Psi_{\left(v_{\phi^{\prime}(h-1)}, w_{\phi^{\prime \prime}(h-1)}\right)_{t},\left(v_{\phi^{\prime}(h-1)+1}, w_{\phi^{\prime \prime}(h-1)}\right)_{t}} \\
& \quad=1^{\otimes(t-1)} \otimes \Psi_{v_{\phi^{\prime}(h-1)}, v_{\phi^{\prime}(h)}} \otimes 1^{\otimes(b-t)},
\end{aligned}
$$

while, in the case of a jump of the 2nd type,

$$
\begin{aligned}
& \Psi_{\left(v_{\phi^{\prime}(h-1)}, w_{\phi^{\prime \prime}(h-1)}\right)_{t},\left(v_{\phi^{\prime}(h)}, w_{\phi^{\prime \prime}(h)}\right)_{t}} \\
& \quad=\Psi_{\left(v_{\phi^{\prime}(h-1)}, w_{\phi^{\prime \prime}(h-1)}\right) t,\left(v_{\phi^{\prime}(h-1)}, w_{\phi^{\prime \prime}(h-1)+1}\right)_{t}} \\
& \quad=\left(\mathbb{1}^{\otimes(1-t)} \otimes \Delta^{\left\{v_{\phi^{\prime}(h-1)}\right\}} \otimes \mathbb{1}^{\otimes(b-t)}\right) \Psi_{w_{\phi^{\prime \prime}(h-1)}, w_{\phi^{\prime \prime}(h)}} .
\end{aligned}
$$

So, we see that, for $d \notin[t, t+a-1]$, we have

$$
\begin{aligned}
& \Psi_{\left(v_{\phi^{\prime}(h-1)}, w_{\phi^{\prime \prime}(h-1)}\right)_{t},\left(v_{\phi^{\prime}(h)}, w_{\phi^{\prime \prime}(h)}\right)_{t}}^{d} \\
& \quad= \begin{cases}1, & \text { at the jump of the 1st type, } \\
\Psi_{w_{\phi^{\prime \prime}(h-1)}, w_{\phi^{\prime \prime}(h)},}^{d}, & \text { jump of the 2nd type, } 1 \leq d \leq t-1, \\
\Psi_{w_{\phi^{\prime \prime}(h-1)},-w_{\phi^{\prime \prime}(h)}}^{d}, & \text { jump of the 2nd type, } t \leq d-a \leq b-1 .\end{cases}
\end{aligned}
$$

We get easily from the definition that

$$
\Delta^{\left\{\left(v_{\phi^{\prime}(h)}, w_{\phi^{\prime \prime}(h)}\right)_{t}\right\}}=\left(\mathbb{1}^{\otimes(t-1)} \otimes \Delta^{\left\{v_{\phi^{\prime}(h)}\right\}} \otimes \mathbb{1}^{\otimes(b-t)}\right) \Delta^{\left\{w_{\phi^{\prime \prime}(h)}\right\}},
$$

consequently,

$$
a^{\left(\left(v_{\phi^{\prime}(h)}, w_{\phi^{\prime \prime}(h)}\right)_{t}, d\right)}= \begin{cases}a^{\left(w_{\phi^{\prime \prime}(h)}, d\right)}, & \text { for } 1 \leq d \leq t-1, \text { and } \\ a^{\left(w_{\phi^{\prime \prime}(h)}, d-a+1\right)}, & \text { for } t \leq d-a \leq b-1 .\end{cases}
$$

Because each $\phi$ has at least one jump of the 1st type, we see that $\xi_{l}$, which should be considered as an element of the normalized bar resolution $\mathcal{B}_{-q-r-s}(V)$, is of the form

$$
\xi_{l}=\sum\left(\alpha_{0}|\ldots| \alpha_{i-1}|1| \alpha_{i+1}|\ldots| \alpha_{q+r+s+1}\right),
$$

for some $1 \leq i \leq q+r+s$, hence it is zero. This also proves the first part of the sublemma.

On the other hand, we have

$$
M_{\pi^{\prime \prime}}^{t}[\tau](\hat{a})=\square_{w_{s}, w_{0}}\left(\sum \bar{\mu}\left(\zeta_{1}\right) \otimes \cdots \otimes \bar{\mu}\left(\zeta_{t-1}\right) \otimes \zeta_{t} \otimes \bar{\mu}\left(\zeta_{t+1}\right) \otimes \cdots \otimes \bar{\mu}\left(\zeta_{b}\right)\right),
$$

with

$$
\begin{aligned}
\zeta_{e}=\sum a_{0}^{\left(w_{0}, e\right)} \otimes \cdots \otimes a_{\pi^{\prime \prime}(1)-1}^{\left(w_{0}, e\right)} \otimes \Psi_{w_{0}, w_{1}}^{e} & \otimes \cdots \otimes \Psi_{w_{s-1}, w_{s}}^{e} \otimes a_{\pi^{\prime \prime}(s)}^{\left(w_{s}, e\right)} \\
& \otimes \cdots \otimes a w_{s} e q+1, \quad 1 \leq e \leq b .
\end{aligned}
$$


Define then $\left(b_{0}\left|b_{1}\right| \cdots \mid b_{q+s+1}\right):=\zeta_{t}$. By Lemma 5.6, for $t \leq l \leq t+a-1$,

$$
\begin{aligned}
&\left(\mathbb{1}^{\odot(t-1)} \odot M_{\pi^{\prime}}^{l-t+1}[\sigma] \odot \mathbb{1}^{\odot(b-t)}\right) M_{\pi^{\prime \prime}}^{t}[\tau](\hat{a}) \\
&=\square_{\left(v_{r}, w_{s}\right)_{t},\left(v_{0}, w_{0}\right)_{t}}\left(\sum\right. \bar{\mu}\left(\zeta_{1}\right) \otimes \cdots \otimes \bar{\mu}\left(\zeta_{t-1}\right) \otimes \bar{\mu}\left(\eta_{1}\right) \otimes \\
& \cdots \otimes \bar{\mu}\left(\eta_{l-t}\right) \otimes \eta_{l-t+1} \otimes \bar{\mu}\left(\eta_{l-t+2}\right) \otimes \\
&\left.\cdots \otimes \bar{\mu}\left(\eta_{a}\right) \otimes \bar{\mu}\left(\zeta_{t+1}\right) \otimes \cdots \otimes \bar{\mu}\left(\zeta_{b}\right)\right)
\end{aligned}
$$

where

$$
\begin{aligned}
\eta_{f}=\sum b_{0}^{\left(v_{0}, f\right)} \otimes \cdots \otimes b_{\pi^{\prime}(1)-1}^{\left(v_{0}, f\right)} \otimes \Psi_{v_{0}, v_{1}}^{f} \otimes \cdots \otimes \Psi_{v_{r-1}, v_{r}}^{f} \otimes b_{\pi^{\prime}(r)}^{\left(v_{r}, f\right)} \\
\otimes \cdots \otimes b_{s+q+1}^{\left(v_{r}, f\right)}, \quad 1 \leq f \leq a .
\end{aligned}
$$

We finish the proof of the sublemma by showing that

$$
\begin{gathered}
\sum \bar{\mu}\left(\zeta_{1}\right) \otimes \cdots \otimes \bar{\mu}\left(\zeta_{t-1}\right) \otimes \bar{\mu}\left(\eta_{1}\right) \otimes \cdots \\
\quad \cdots \otimes \bar{\mu}\left(\eta_{l-t}\right) \otimes \eta_{l-t+1} \otimes \bar{\mu}\left(\eta_{l-t+2}\right) \otimes \\
\quad \cdots \otimes \bar{\mu}\left(\eta_{a}\right) \otimes \bar{\mu}\left(\zeta_{t+1}\right) \otimes \cdots \otimes \bar{\mu}\left(\zeta_{b}\right)
\end{gathered}
$$

is the same as

$$
\sum \bar{\mu}\left(\xi_{1}\right) \otimes \cdots \otimes \bar{\mu}\left(\xi_{l-1}\right) \otimes \xi_{l} \otimes \bar{\mu}\left(\xi_{l+1}\right) \otimes \cdots \otimes \bar{\mu}\left(\xi_{a+b-1}\right) .
$$

Observe first that the terms at the $d$-th place for $d \notin[t, t+a-1]$ agree. Indeed, for such $d$ put

$$
g(d):= \begin{cases}d, & \text { for } 1 \leq d \leq t-1, \text { and } \\ d-a+1, & \text { for } t \leq d-a \leq b-1\end{cases}
$$

It is clear from the above considerations that

$$
\begin{aligned}
\bar{\mu}\left(\xi_{d}\right)=\bar{\mu}\left(\sum a_{0}^{\left(w_{\phi^{\prime \prime}(0)}, g(d)\right)} \otimes \cdots \otimes a_{\pi(1)-1}^{\left(w_{\phi^{\prime \prime}(0)}, g(d)\right)} \otimes X_{1} \otimes\right. \\
\left.\cdots \otimes X_{r+s} \otimes a_{\pi(r+s)}^{\left(w_{\phi^{\prime \prime}(r+s)}, g(d)\right)} \otimes \cdots \otimes a_{q+1}^{\left(w_{\phi^{\prime \prime}(r+s)}, g(d)\right)}\right)
\end{aligned}
$$

with

$$
X_{h}:= \begin{cases}1, & \text { at the jump of the 1st type, and } \\ \Psi_{w_{\phi^{\prime \prime}(h-1)}, w_{\phi^{\prime \prime}(h)}}^{g(d)}, & \text { at the jump of the 2nd type. }\end{cases}
$$

Since the multiplication - on $V$ is supposed to be unital, we may simply "forget" all the $X_{h}$ 's at the jumps of the first type. The claim that $\bar{\mu}\left(\xi_{d}\right)=\bar{\mu}\left(\zeta_{g(d)}\right)$ is now obvious. To show the equality of the terms at the $d$-th place for $d \in[t, t+a-1]$ is even easier, as we see immediately that for such $d, \xi_{d}=\eta_{d-t+1}$. This finishes the proof of the sublemma "modulo the signs". It remains to show that

$$
\operatorname{sgn}\left(\pi, \sigma \diamond_{t, \phi} \tau\right) \cdot \operatorname{sgn}(\phi)=(-1)^{(a+1) s+(b+1) r} \cdot \operatorname{sgn}\left(\pi^{\prime}, \sigma\right) \cdot \operatorname{sgn}\left(\pi^{\prime \prime}, \tau\right)
$$

which is, by the definition of $\operatorname{sgn}(-,-)$, the same as congruence modulo 2

$$
\begin{aligned}
o(\pi) & +(a+b-1)(r+s)+\sum \hat{i}_{a} \\
& \equiv(a+1) s+(b+1) r+o\left(\pi^{\prime}\right)+a r+o\left(\pi^{\prime}\right)+b s .
\end{aligned}
$$

All the terms involving $a, b, r, s$, cancel leaving $o(\pi)+\sum \hat{i}_{a}=o\left(\pi^{\prime}\right)+o\left(\pi^{\prime \prime}\right)$, which follows immediately from the construction of $\pi$ from $\pi^{\prime}$ and $\pi^{\prime \prime}$ as described before the statement of Sublemma 5.11. 
This completes the proofs of the technical sublemmas and thus we have established conclusively the existence of the permanent paranormal object described in Theorem 5.1. See $[1$, p. 200] for the terminology.

\section{REFERENCES}

1. J. Beloff, The relentless question. Reflections on the paranormal, McFarland Publishers, NC, USA, 1990.

2. P. Cartier, Cohomologie des coalgèbres, Séminaire Sophus Lie 1955-1956, exp. 4\&5.

3. V.G. Drinfel'd, Quasi-Hopf algebras, Algebra i Analiz, 1 (1989), no. 6, 114-148; English transl. in Leningrad Math. J. 1 (1990). MR 91b:17016

4. T.F. Fox, An introduction to algebraic deformation theory Journal of Pure and Appl. Algebra, 84 (1993), 17-41. MR 93k:16055

5. M. Gerstenhaber, On the deformation of rings and algebras, I Annals of Mathematics, 79 (1964), 59-104; II, 84 (1966), 1-19; III, 88 (1968), 1-34; IV, 99 (1974), 257-276. MR 30:2034; MR 34:7608; MR 39:1521; MR 52:10807

6. M. Gerstenhaber, The cohomology structure of an associative ring, Annals of Mathematics, (2) 78 (1963), 268-288. MR 28:5102

7. M. Gerstenhaber and S.D. Schack, Algebraic cohomology and deformation theory, In Deformation Theory of Algebras and Structures and Applications, pages 11-264. Kluwer, Dordrecht, 1988. MR 90c: 16016

8. M. Gerstenhaber and S. D. Schack, Bialgebra cohomology, deformations, and quantum groups, Proc. Nat. Acad. Sci., 87 (1990), 478-481. MR 90j:16062

9. S. Mac Lane, Homology, Springer-Verlag, 1963. MR 30:1160

10. M. Markl, Cotangent cohomology of a theory and deformations, Journal of Pure Appl. Algebra, to appear.

11. M. Markl, Models for operads, Submitted. Available as preprint hep-th/9411208.

12. M. Markl and S. Shnider, Drinfel'd algebra deformations and the associahedra, IMRN, 1994, no. 4. Math. Journal, submitted. MR 95f:16050;

13. M. Markl and J.D. Stasheff, Deformation theory via deviations, Journal of Algebra, 70 (1994), 122-155. CMP 95:04

14. S. Shnider and S. Sternberg, The cobar construction and a restricted deformation theory for Drinfeld algebras, Journal of Algebra, 169 (1994), 343-366. CMP 95:02

15. S. Shnider and S. Sternberg, Quantum groups: from coalgebras to Drinfeld algebras, Graduate texts in mathematical physics, ed. Elliot Lieb, International Press, 1993. MR 95e:17022

16. J.D. Stasheff, Homotopy associativity of H-spaces I, II, Trans. Amer. Math. Soc., 108 (1963), 275-312. MR 28:1623

Mathematical Institute of the Academy, Žitná 25, 11567 Praha 1, Czech Republic

E-mail address: mark@earn.cvut.cz

Department of Mathematics, Bar-Ilan University, Ramat-Gan, Israel

E-mail address: shnider@bimacs.cs.biu.ac.il 